\title{
A Highly Abstracted Method of FPGA-Based Development FOR SECONDARY SURVEILlanCE RADAR TRANSPOND DETECTION
}

\author{
A Project Presented to \\ the Faculty of California Polytechnic State University, \\ San Luis Obispo
}

\author{
In Partial Fulfillment \\ of the Requirements for the Degree \\ Master of Science in Electrical Engineering
}

by

James Penn Watt

July 2009 
(C) 2009

James Penn Watt

All Rights Reserved 


\section{COMMITTEE MEMBERSHIP}

TITLE:

A Highly Abstracted Method of FPGA-Based Development for Secondary Surveillance Radar Transpond Detection

AUTHOR:

James Penn Watt

DATE SUBMITTED:

28 July 2009

COMMITTEE CHAIR:

Dennis Derickson, Assistant Professor, Graduate Coordinator

COMMitTeE MEMBER:

James Harris, Professor

COMMitTeE MeMBeR:

Fred DePiero, Professor, Associate Dean 


\begin{abstract}
A Highly Abstracted Method of FPGA-BASED DeVElopment FOR SECONDARY SURVEILlANCE RADAR TRANSPOND DETECTION

James Penn Watt
\end{abstract}

Traditional FPGA-based digital design is based on writing hardware definition language (HDL) code from scratch. Time to market, cost of development, and the level of training required for designers all can be reduced with a simplified and abstracted design strategy. This project intends to demonstrate a graphical user interface (GUI) layer of abstraction on top of existing commercially produced design aids including MATLAB, Simulink, and Xilinx System Generator. This project performs and demonstrates a specific implementation example of a Secondary Surveillance Radar (SSR) message decoder as proof-of-concept for the abstracted design method. The abstracted digital design methods shown in this project can be adapted for use in other areas of development and research including digital signal processing and communications.

Keywords: Digital Design, HDL, IFF, Mode S, SSR, ViaSat 
I would like to thank my project advisor, Dennis Derickson, Ph.D., for his guidance and initiative that helped revive this project through cooperation with ViaSat and carried on throughout the course of the past year. Thank you to ViaSat, Inc., for their project sponsorship and originating this cooperation with Cal Poly. Special thanks also go to Jeff Rowley from ViaSat for his technical direction and availability despite the long distance.

Thank you also to my project teammates Robert Oxsen, Wesley Lilienthal, and Jason Lincoln for their dedication to their individual facets of this project. 


\section{TABLE OF CONTENTS}

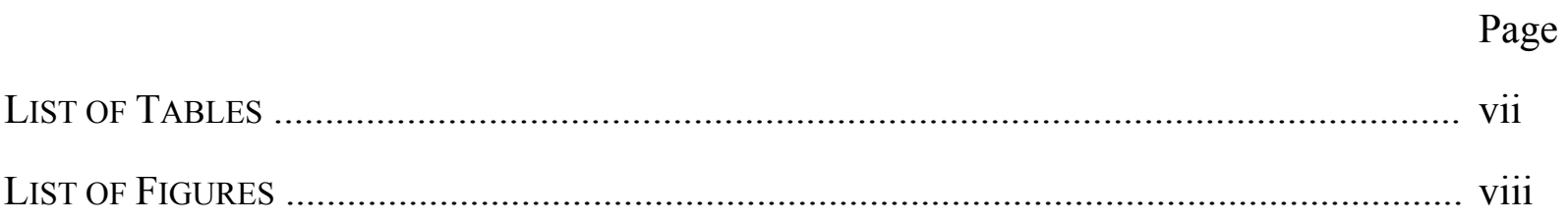
CHAPTER

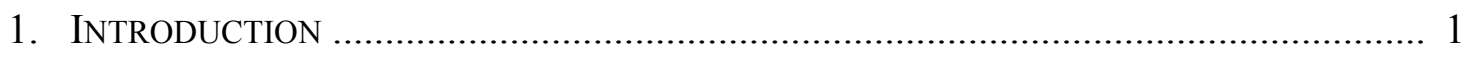

1.1. Typical HDL Design Approach ........................................................ 1

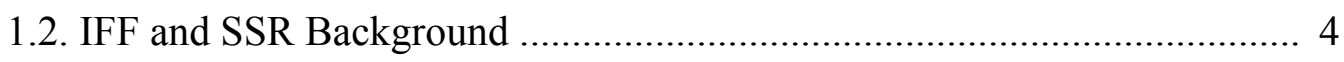

1.3. Problem Definition and Requirements ............................................. 8

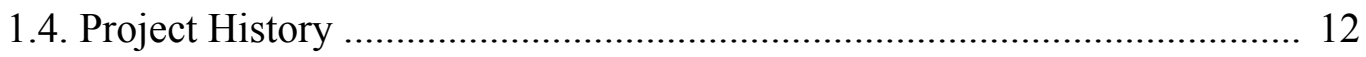

2. DESIGN AND IMPLEMENTATION ................................................................... 18

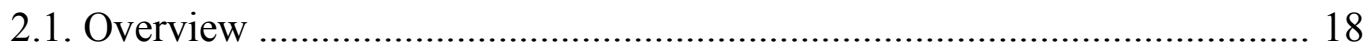

2.2. Front-End GUI for Waveform Definition ......................................... 20

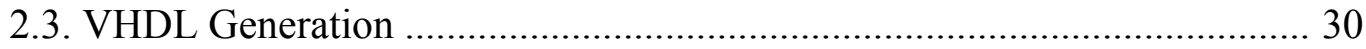

2.4. Hardware Platform .............................................................................. 33

2.5. System Integration in Xilinx Platform Studio ...................................... 36

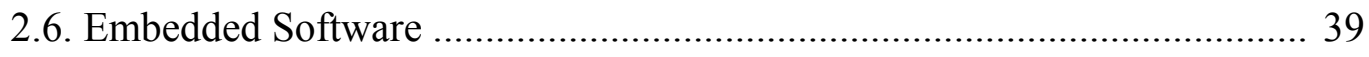

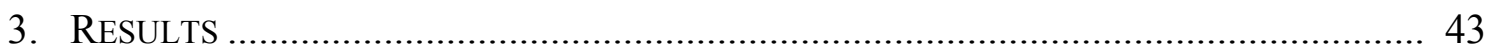

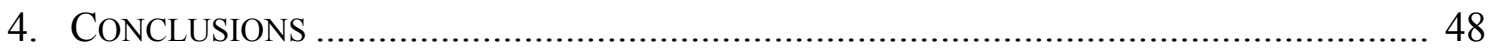

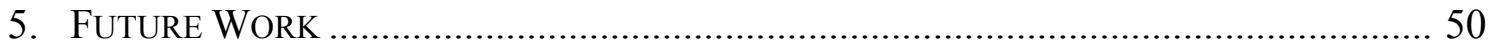

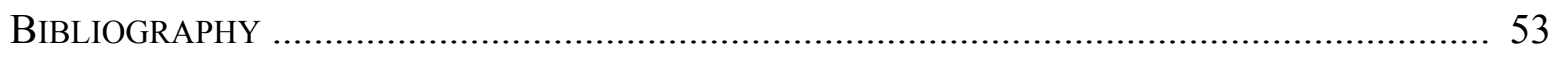

APPENDICES

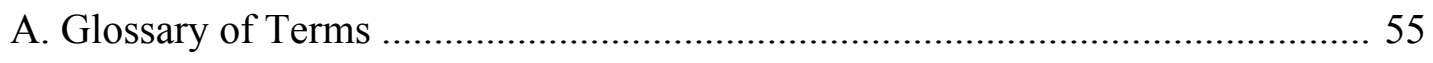

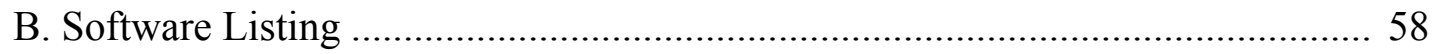

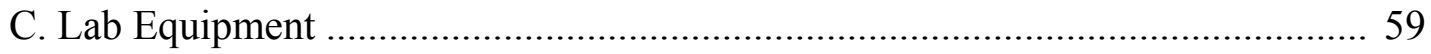

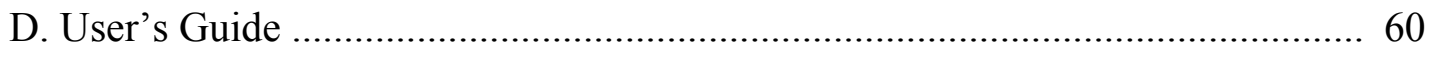




\section{LIST OF FIGURES}

Figure $\quad$ Page

1.1 Xilinx System Generator Design Flow Overview ...................................... 3

1.2 IFF Interrogation and Transpond System Overview .................................... 5

1.3 Mark XII Mode A/C and Mode S Transponds ................................................. 7

1.4 Simulated Aircraft RF Environment with IFF Transponder Pulses Shown .......... 9

1.5 Aircraft Testing in the Benefield Anechoic Facility at Edwards Air Force Base .... 10

1.6 Complete Detector System Overview Flowchart .......................................... 11

1.7 IFF Mark XII, Mode A/C Transpond Waveform ........................................... 13

$1.8 \quad$ Original Detector Implementation State Diagram ......................................... 14

1.9 Simulink Model Containing Xilinx System Generator Elements and Detector M-Code Block .......................................................................... 15

1.10 Lab Equipment and Development Platform Used by 2008 Senior Project Students .......................................................................... 16

1.11 Oscilloscope Screen-Capture Showing IFF Mark XII Mode A/C Framing Pulses (Top) and a Detected Indicator Signal (Bottom) .................................... 17

2.1 Overview of the Detector Design and Implementation Process .......................... 19

2.2 IFF Mark XII Mode S Waveform, Shown as an Example of What Can be Defined and Saved by the Waveform Definition GUI .................................... 21

2.3 The Custom Waveform Definition GUI ....................................................... 22

2.4 State 0, Waiting for the First Rising Edge of the Frame to Trigger the Detector FSM

2.5 Framing Pulse Detection Block Flowchart ................................................ 24

2.6 Pulse-Position Modulated (PPM) Data Pulse Block Flowcharts .......................... 25

2.7 On-Off Keyed (OOK) Data Pulse Block Flowchart ....................................... 26

2.8 Desired Output Stage Flowchart, Common to All Detector Implementations ....... 27

2.9 Writing to the FIFO During Pulse Detection Is Not Ideal, But Circumvents a Bug 
2.10 Simulink Model Used by Xilinx System Generator to Perform Testing and Write VHDL Code

2.11 The Detector Design Is Converted from a Graphical Representation to M-Code to HDL Code

2.12 Block Diagram of the ML410 Development Board's Subsystems ....................... 34

2.13 Schematic Diagram and Photograph of the Two SMA High Speed Clock

Interface Pins Used as Input and Output Ports .................................................. 35

2.14 System Integration Takes Place within the Xilinx Platform Studio Environment .. 37

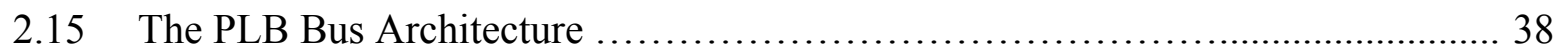

2.16 The VxWorks Real-Time Operating System Performs Additional

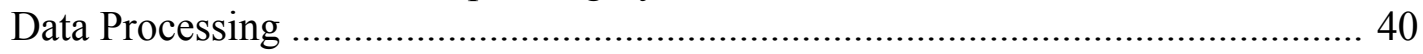

2.17 The VxWorks Splash Screen Displayed Over the Serial Interface ....................... 41

3.1 Oscilloscope Output Showing IFF Mark XII Mode S Extended

Squitter Transpond and "Matched" Flag

3.2 Oscilloscope Output Showing IFF Mark XII Mode S Message

$+7 \%$ Out of Specification and No "Matched" Flag

3.3 Oscilloscope Output Showing IFF Mark XII Mode S Message

$+5 \%$ Out of Specification and "Matched" Flag 46

3.4 VxWorks Terminal Output Showing Detected IFF Mark XII Mode S Messages ... 47 


\section{INTRODUCTION}

This project looks at potential tools and means to abstract the digital design process above the level of writing code by hand. An abstracted process can reduce turnaround times for development, reduce costs, and make digital design possible for technicians and engineers not trained in traditional FPGA-based digital design methods. Specifically, this project looks at implementing a detector for Secondary Surveillance Radar (SSR) transpond messages by way of creating a specialized GUI-based design environment in MATLAB which interfaces with Xilinx System Generator software. Then, the detector designed in the graphical environment is integrated into a larger hardware system within a Xilinx development board.

\subsection{Typical HDL Design Approach}

FPGA-based digital hardware design typically revolves around first developing a design in a higher level programming language such as MATLAB or $\mathrm{C} / \mathrm{C}++$ and performing initial simulations in this higher level environment. Then, developers write hardware description language (HDL) code by hand to implement the design in digital hardware. The two most prolific languages used for FPGA-based digital design, VHDL and Verilog, are written into modules, synthesized, then mapped to hardware by specialized programs that reduce logic, improve timings, and optimize area, layout, speed, and power. In addition to custom-written HDL modules, designers often have the choice of integrating prewritten intellectual property (IP) cores into a design. Designers use IP cores as modular components in part of a larger design, saving the effort and time of writing and debugging these components. When 
building a large and complicated design, abstraction eases the task of writing the HDL code by breaking up the design into multiple sub-modules, using prewritten IP cores, and allowing the synthesizer to remap logic.

In contrast to the two-stage traditional development approach (higher level language simulation and manual HDL composition), it is possible to use software tools to automatically move from a higher level language implementation to HDL. In an effort to create another layer of abstraction, digital design companies have started producing tools that help designers back away from the process of writing HDL by hand. One such tool, Xilinx System Generator, runs within the MATLAB and Simulink environment and "presents a high level abstract view of a DSP system, yet nevertheless automatically maps the system to a faithful hardware implementation" [1]. As shown in Figure 1.1, Xilinx System Generator converts a Simulink model created with special Xilinx logic blocks into IP cores, VHDL, control signals, and supporting data used for synthesis, mapping, and routing. 


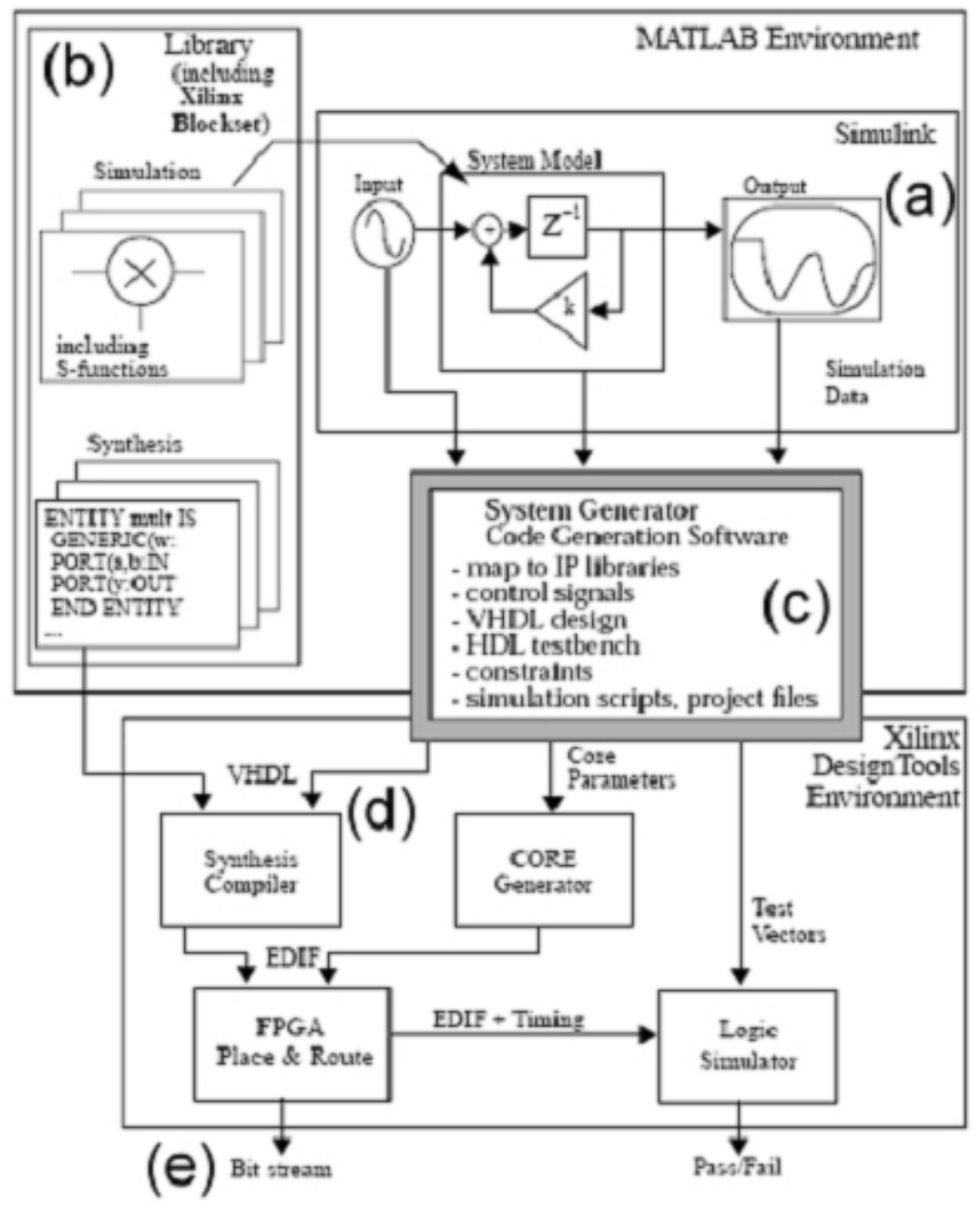

Figure 1.1: Xilinx System Generator Design Flow Overview [1]

(a) The MATLAB and Simulink environments hold the higher level language design.

(b) Libraries provide additional "black box" modules to the design.

(c) Xilinx System Generator software converts higher level language code to HDL. (d) HDL output from Xilinx System Generator.

(e) Complete hardware design after HDL synthesis, ready to be used in a device.

This project goes a step beyond the level of abstraction provided by Xilinx System Generator alone by removing the need for hand written programming in both the higher level language and HDL environments. A graphical abstraction layer on top of the higher level language programming serves as the user interface for developing an algorithm to be implemented in hardware. Based on inputs provided in 
the graphical environment, an export function generates MATLAB M-code which is then converted by Xilinx System Generator into HDL code.

\subsection{IFF and SSR Background}

Identification, Friend or Foe (IFF) was developed in its original form during World War II [2]. Secondary Surveillance Radar (SSR) and the Air Traffic Control Radar Beacon System (ATCRBS) are the civilian counterparts to IFF developed soon after [3]. Primary radar bounces radar pulses off physical objects and can only detect the presence of aircraft, not any data about who the aircraft are or what nation they belong to. Clearly, in a wartime setting, identification data is essential to knowing whether a target is friendly or hostile. In air traffic control situations, identification data is also very important to routing aircraft and keeping the skies safe for passengers. Secondary radar provides identification and position data beyond the capabilities of primary radar, including information such as country of origin and altitude. Secondary radar systems such as IFF/SSR are active identification and air traffic control communication systems and are comprised of a two-way communication between aircraft and/or ground stations.

The IFF communications scheme consists of two parts: the interrogation (uplink) and the transpond (downlink). The most recent incarnation of IFF communications is known as Mode S. The two common Modes of IFF communication developed prior to Mode S are Mode A and Mode C. IFF Mark XII Mode A provides identification data while Mode $\mathrm{C}$ provides altitude information based on a pressure reading [4]. 
Figure 1.2 shows the two-way IFF communications system overview. Prior to Mode $\mathrm{S}$, these messages were all multicast, meaning messages were sent to no specific recipient, and any transponder that received an interrogation would respond [5]. In

Mode S, messages can be addressed to individual recipients by using a 24-bit International Civil Aviation Organization (ICAO) Aircraft Address [6].

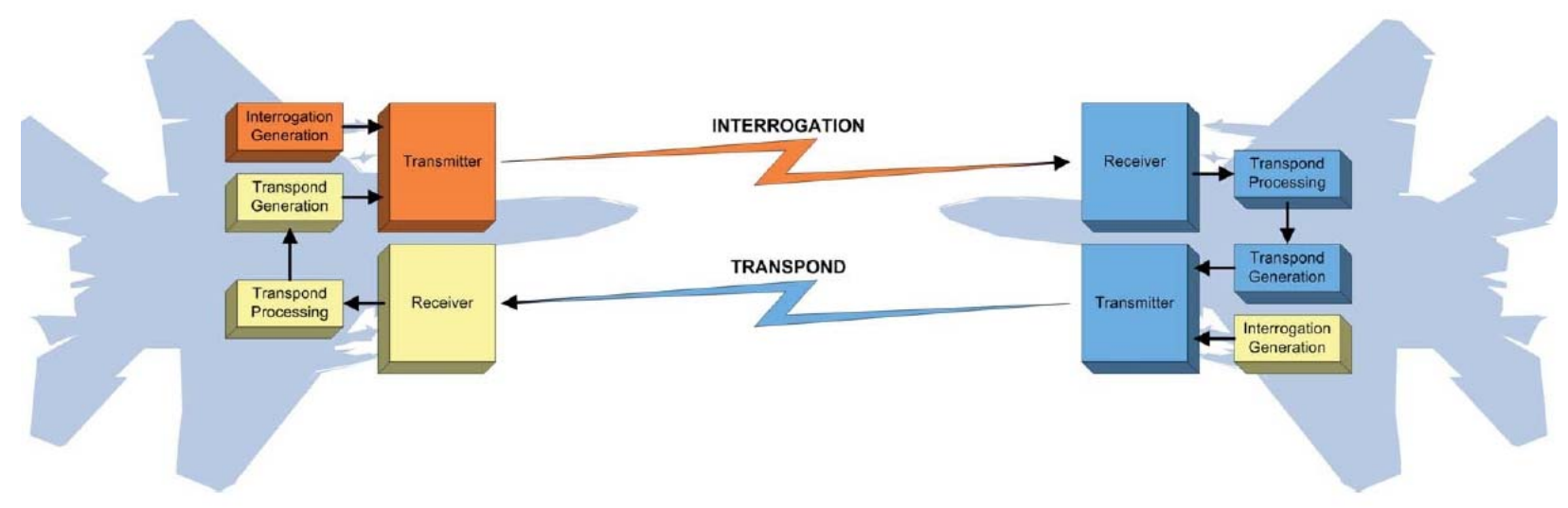

Figure 1.2: IFF Interrogation and Transpond System Overview [7]

IFF communications are two-way: first an interrogation is sent (orange arrow), then the recipient responds with a transpond (blue arrow). This project focuses on detecting, demodulating, and interpreting the transpond message.

Mode S was developed between 1961 and 1975 at MIT's Lincoln Labs as an improvement to the SSR system that seamlessly integrated with the existing infrastructure [4]. In 1961, President John F. Kennedy commissioned reports that would outline the future of United States air traffic control and integration between civilian and military air traffic control systems - one of these reports was known as Project Beacon [8]. Project Beacon addressed the development strategy of air traffic control technology amidst safety concerns following the 1960 midair collision of a United Airlines DC-8 aircraft and a TWA Super Constellation over Staten Island, 
New York [4], [9]. Mode S was slowly developed in line with the recommendations of Project Beacon as a solution to these safety concerns and is designed to be backwards compatible with Mode A and Mode C messages [4].

Mode $\mathrm{S}$ is addressable, meaning messages can be sent to a specific receiver address rather than multicast to anyone in "earshot". The addressability of Mode S helps alleviate one of the problems found in previous IFF schemes, false replies uncorrelated in time (FRUIT). FRUIT occurs when several aircraft respond to the same IFF interrogation and their replies overlap and become garbled [10]. This unwanted interference is especially a problem in areas of tightly packed flight patterns, such as over the European Union. Addressable IFF interrogations eliminate the need for multicasting beyond the first message, reducing the total number of messages received by transponders and alleviating the problem of FRUIT. Occasional multicast messages are still required to ascertain the addresses of aircraft in range. The reliability of messages is increased in Mode $\mathrm{S}$ due to a parity check field contained in the data section of Mode S messages [11]. Mode S also increases the precision of altitude measurements from 100 feet to 25 feet increments [12]. 
(a)

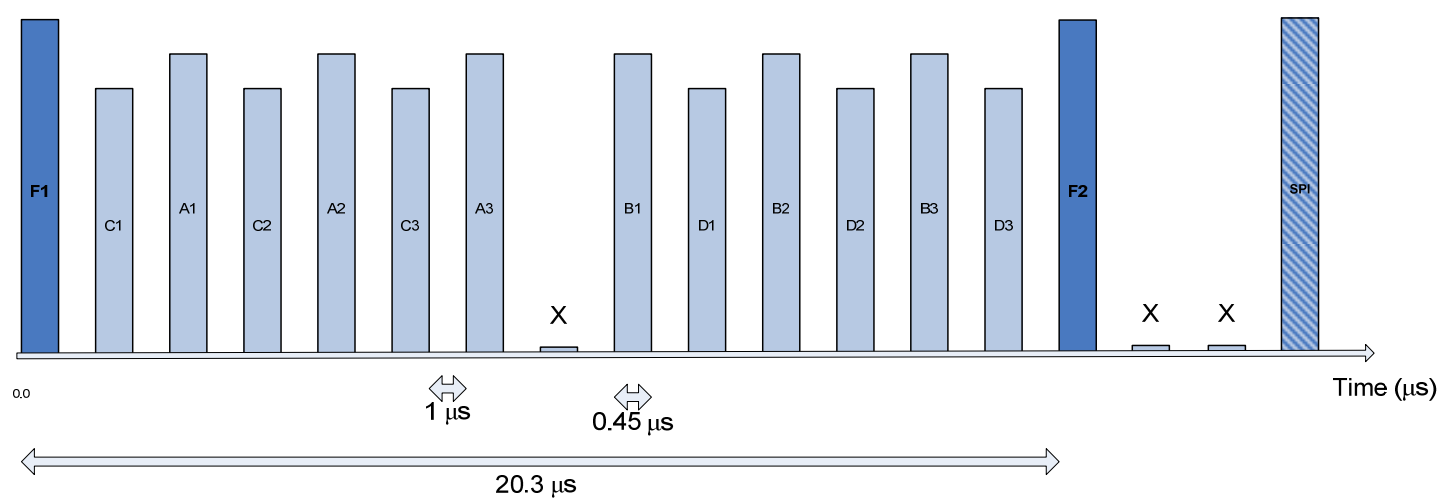

(b)

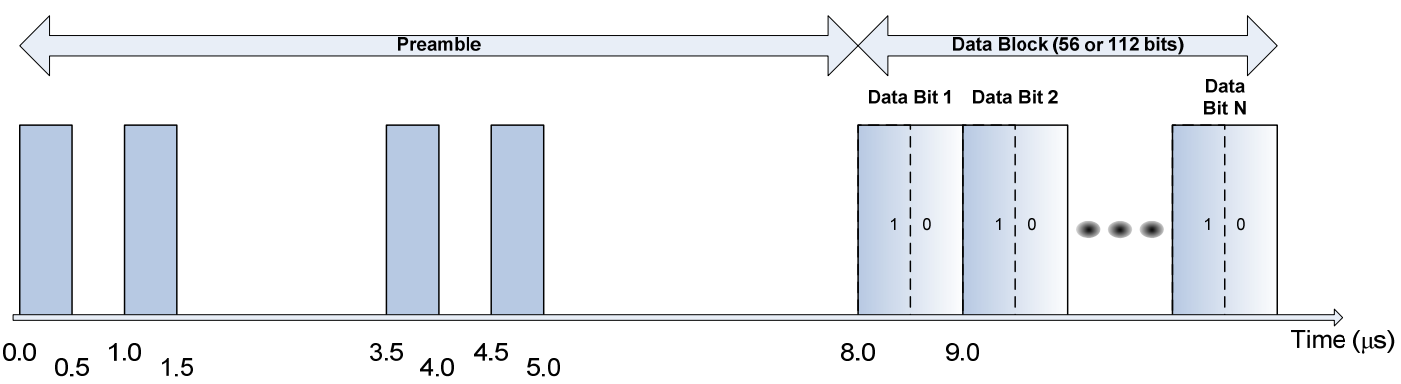

Figure 1.3: IFF Mark XII Mode A/C (a) and Mode S (b) Transponds [11]

The Mode A/C transpond (a) is an older format and is limited to 12 bits of data. The Mode $S$ format (b) is the primary test waveform in this project and can contain 56 or 112 bits of data, modulated in a Pulse Position Modulation (PPM) scheme.

Figure 1.3 compares the waveforms associated with IFF Mark XII Mode A/C and Mode S. Section 1.4 describes a 2008 Cal Poly senior project by James Wu and Ivan Bland and goes into more detail regarding the Mode $\mathrm{A} / \mathrm{C}$ waveform and their detector solution. Mode $\mathrm{S}$ interrogations and transponds are sent on the 1030 and $1090 \mathrm{MHz}$ bands, respectively [13]. Mode S pulses have a pulse width of $0.5 \mu$ s each, making the corresponding data pulse window $1.0 \mu$ s wide [11]. The Mode S waveform begins with four framing pulses, known as the preamble, which do not explicitly contain data but are required for a transpond to be properly formatted [11]. The following 56 or 112 pulses contain data encoded in a Pulse Position Modulation (PPM) scheme - a 
pulse residing in the first half of the pulse window corresponds to a data bit of 1 , while a pulse residing in the second half of the pulse window corresponds to a data bit of 0 . The shorter data bloc of 56 bits is the traditional Mode $\mathrm{S}$ message length and the 112 bit data block is known as Extended Squitter. The encoded data can include information such as an aircraft's identification or altitude, and contains a parity check field to ensure reliability [11]. The longer data field of Mode S, compared to Modes $\mathrm{A}$ and $\mathrm{C}$, makes the IFF communication channel more robust. Mode $\mathrm{S}$ is the latest iteration of the constantly evolving IFF/SSR communications protocol and is used on modern aircraft, making Mode $\mathrm{S}$ a prime candidate for testing and verification of a detector design solution.

\subsection{Problem Definition and Requirements}

This project is sponsored by ViaSat, Inc., a company that provides "innovative satellite and other digital communication products that enable fast, secure, and efficient communications to any location" [14]. One of the ViaSat products is a complete test and simulation environment for aircraft radios including IFF transponders [15]. The ViaSat test and simulation environment is depicted in Figure 1.4. ViaSat's test solution reproduces the radio environment that an aircraft might experience while in flight - but a missing piece is the ability to listen for and decode IFF transponds generated by the system under test (SUT). 


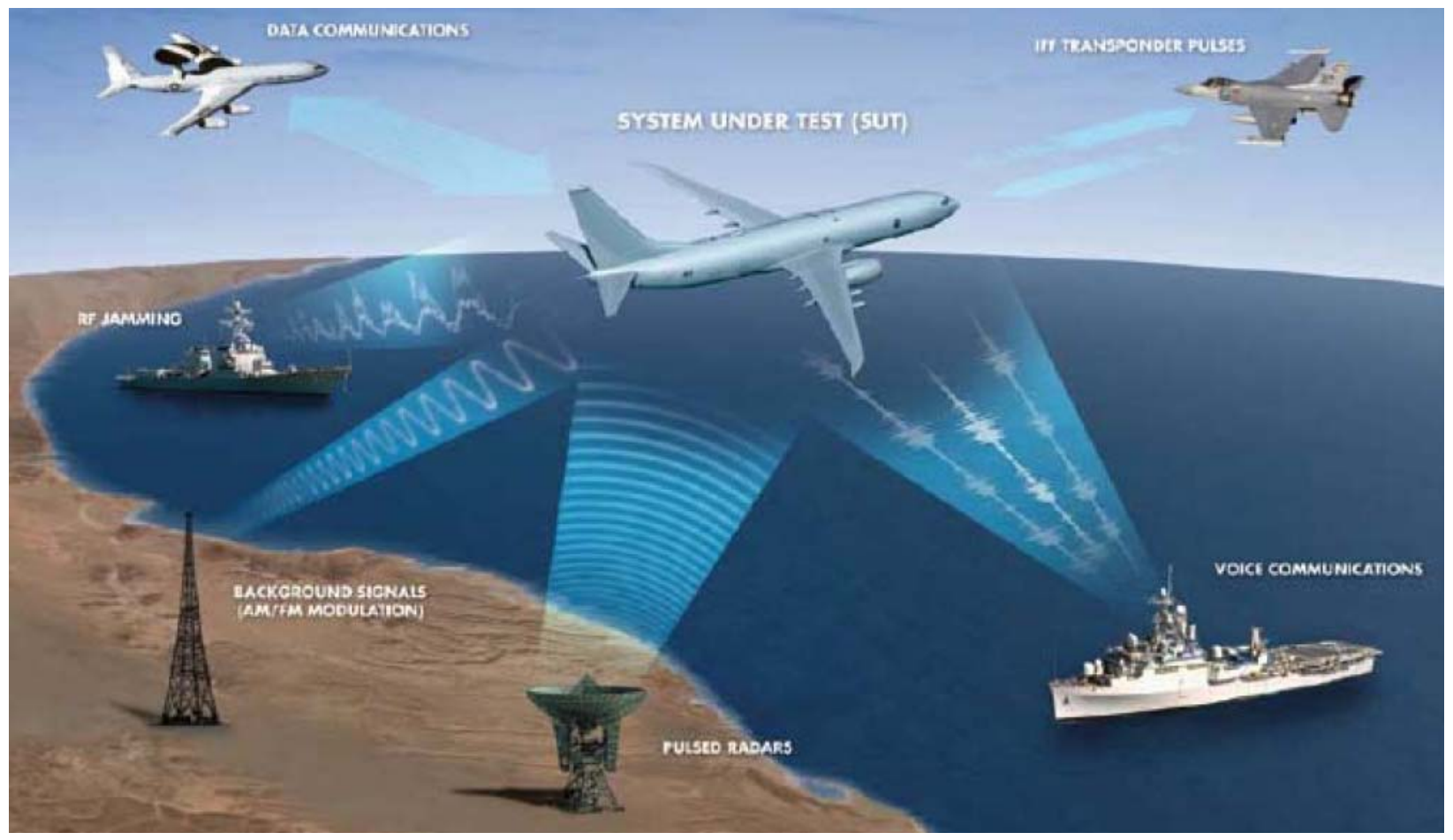

Figure 1.4: Simulated Aircraft RF Environment with IFF Transponder Pulses Shown [15] In the ViaSat test solution, the System Under Test (SUT) is stimulated with a variety of artificial $R F$ sources including IFF transponder pulses. ViaSat would like this system to be able to listen for and interpret the SUT's replies to IFF interrogations.

Aeronautical communications testing systems are employed so that the radio equipment on board an aircraft can be tested while the aircraft is still on the ground. Aircraft in such a test environment are separated from outside radio interference and an anechoic chamber simulates a test environment without reflective walls surrounding the SUT. The largest anechoic test facility of this type is located at Edwards Air Force Base in California [16], shown in Figure 1.5. Many different communication waveforms (including IFF) as well as jamming, radar pulses, and other RF sources are produced in the test environment by ViaSat equipment [15]. 


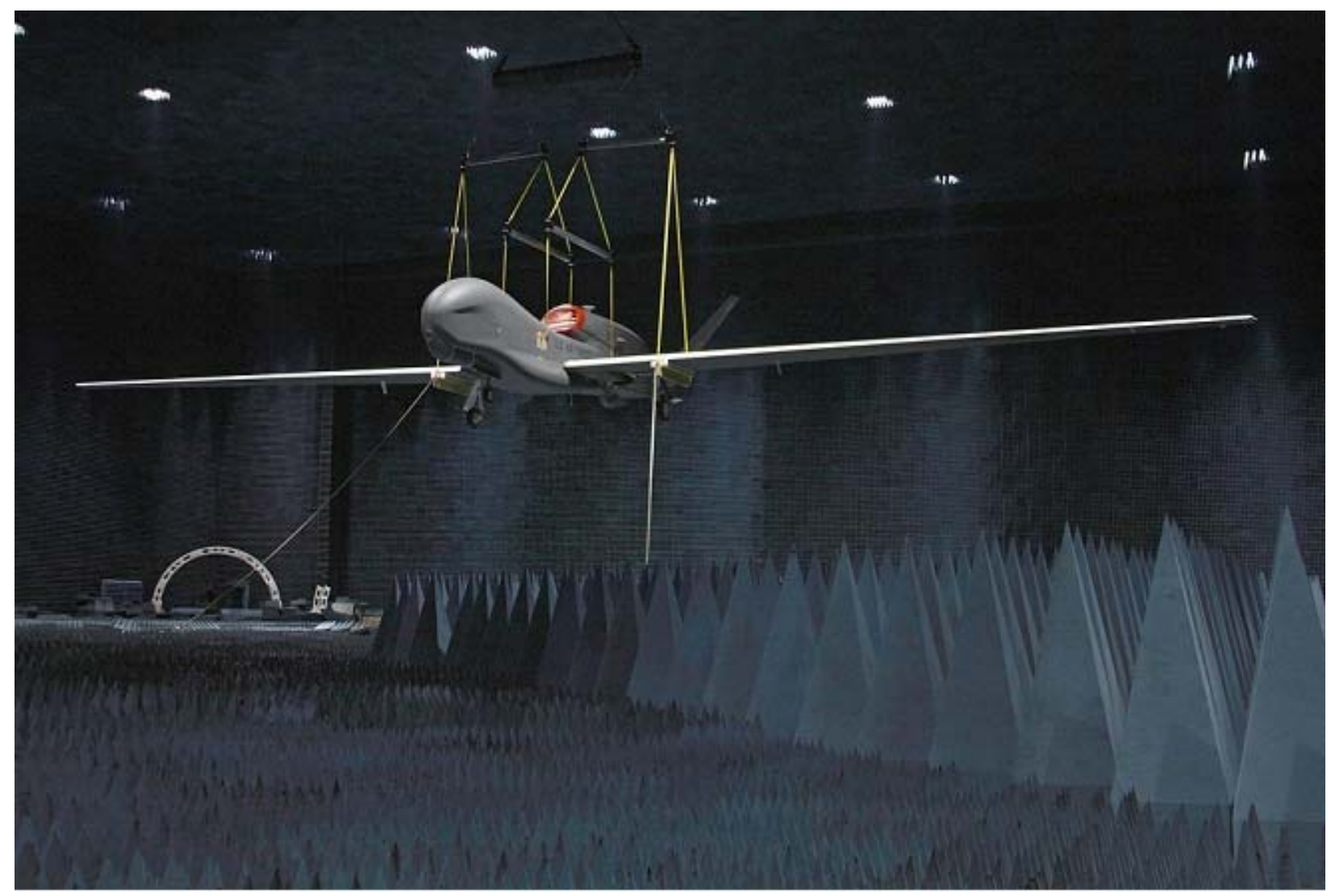

Figure 1.5: Aircraft Testing in the Benefield Anechoic Facility at Edwards Air Force Base [17] ViaSat test equipment is used in aircraft testing facilities including the Benefield Anechoic Facility at Edwards Air Force Base. An entire RF environment is simulated so that the aircraft's radios undergo a similar experience to actual flight or combat situations.

The development of Mode S exemplifies the changing nature of the IFF system and consequently, test and simulation systems that utilize IFF must also adapt to fit the new technology. ViaSat requested a system for detection of IFF data pulses and a means of extracting the encoded data within each pulse. IFF Mark XII Mode S was chosen as the specific proof-of-concept target waveform, but ViaSat wanted a design process that was simplified beyond the level of handwritten HDL code. The abstracted design process would reduce the design time for various IFF waveform detectors and also reduce the required training and experience of the designer 
necessary to produce new detector hardware designs. The simplified design allows the designer to make quick and easy updates to their detectors and adapt detectors to listen for new waveforms with minimal effort. Based on the requirements and desired features expressed by ViaSat, we developed the system overview flowchart for our project, shown in Figure 1.6.

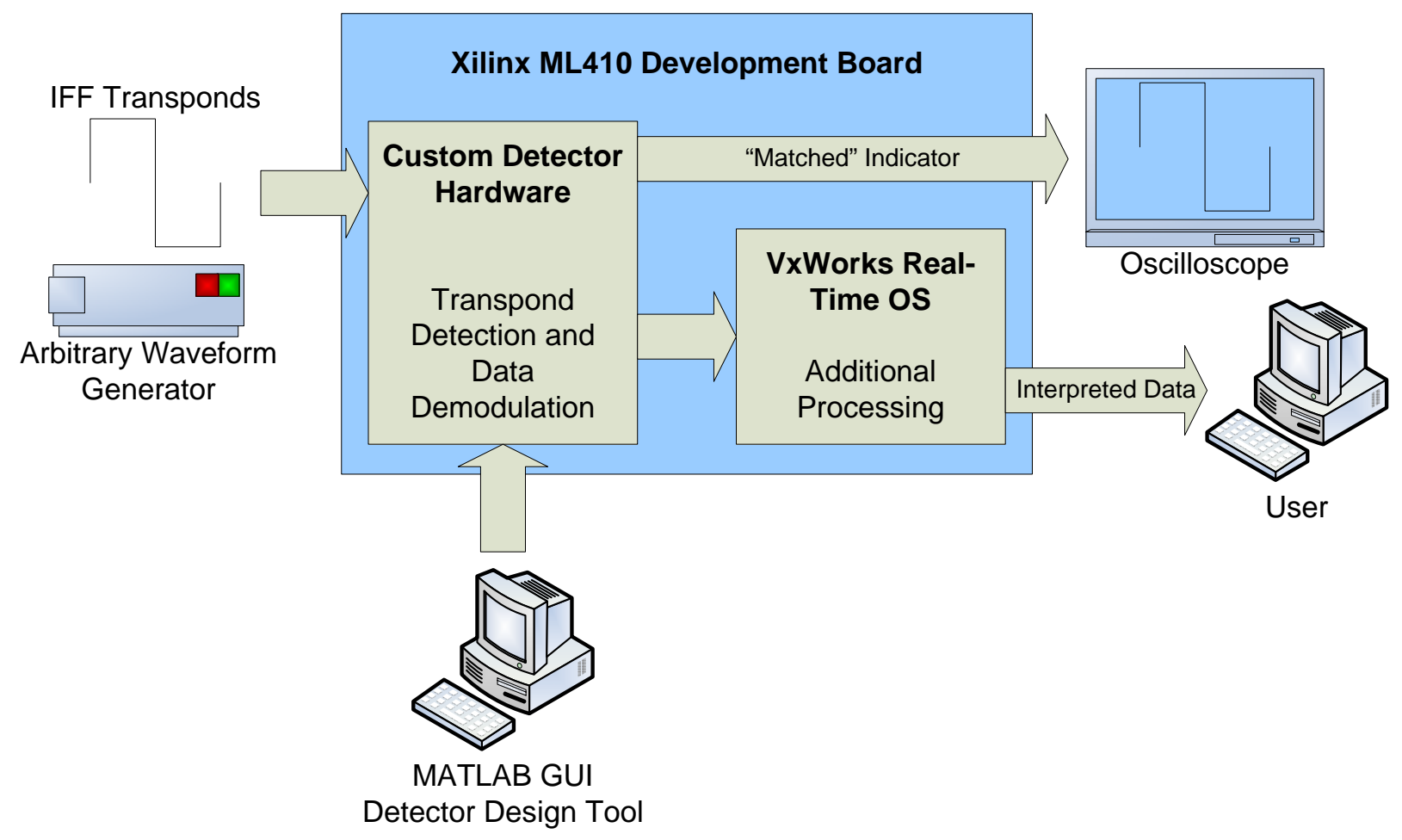

Figure 1.6: Complete Detector System Overview Flowchart

IFF transponder pulses are first validated and data is demodulated by custom hardware programmed into the FPGA fabric. The demodulated data is passed to the VxWorks system running additional custom processing software that converts a bitstream of data into a meaningful, human-readable message. The message data is then sent to the user over Ethernet.

For the sake of simplicity, design of the detector hardware is done in a custom MATLAB GUI and exported to HDL code. Sections 1.1, 2.2, and 2.3 go into more detail about the benefits of the graphical HDL design process as well as 
implementation details. The Xilinx ML410 board serves as the hardware platform for the detector and supporting peripherals, software, and I/O. The details of the ML410 system are described in Section 2.4. Within the fabric of the Xilinx FX60 Virtex-4 FPGA on the ML410 board are the detector hardware and a VxWorks real-time operating system running on a PowerPC processor. The detector performs IFF transpond validation and data demodulation while additional processing in software on the VxWorks system converts messages to human-readable formats and sends these messages to a user over Ethernet. A "matched" indicator is sent from the detector hardware out to an oscilloscope to indicate a message has been received and data has been extracted.

\subsection{Project History}

Collaboration between Cal Poly and ViaSat on this project began in 2008 with a senior project by James Wu and Ivan Bland [18]. This first iteration of the project detected the framing bits of an IFF Mark XII Mode A/C transpond. The detector circuit validated the pulse widths and spacing between pulses F1 and F2, shown in Figure 1.7. 


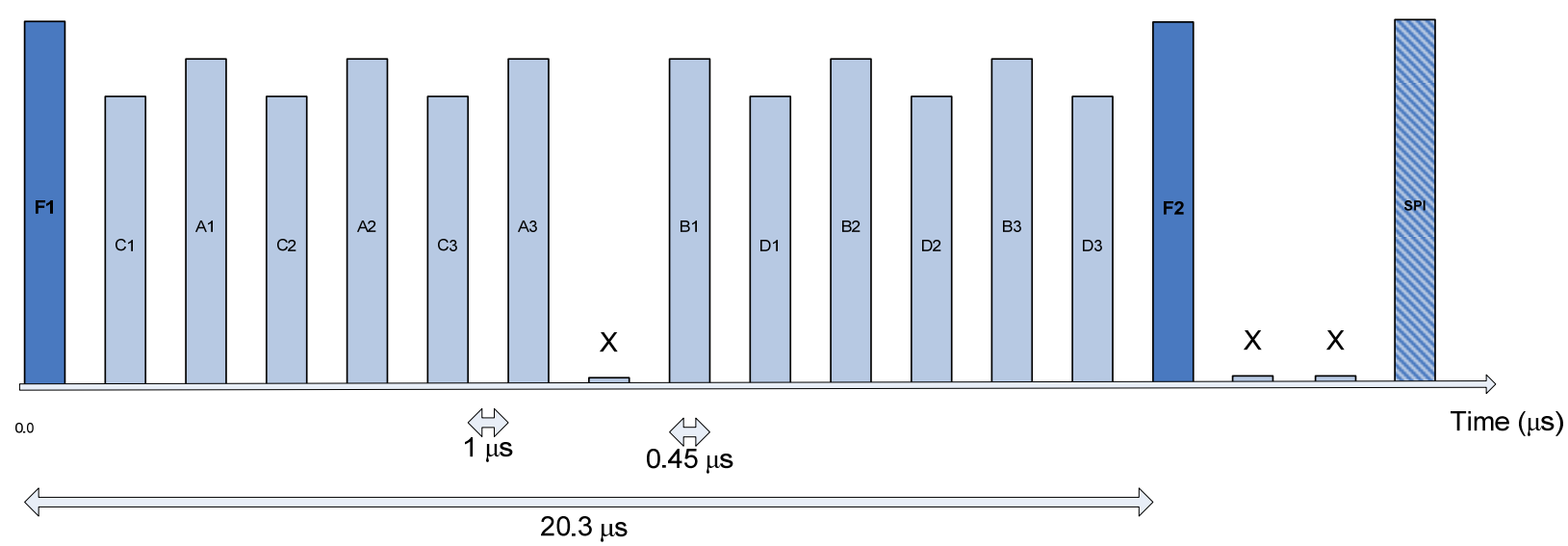

Figure 1.7: IFF Mark XII, Mode A/C Transpond Waveform [11] Framing pulses $F 1$ and $F 2$ are shown in dark blue, $X$ 's represent empty pulse windows, and the light blue pulses contain data. The final pulse, Special Position Identification (SPI), is used in special position identification messages.

The original senior project implementation centers on using Xilinx System Generator, MATLAB, and Simulink to implement the Mode A/C detector design in VHDL and, ultimately, on the Xilinx ML410 development board as custom hardware. The waveform detector is a finite state machine (FSM) handwritten in MATLAB Mcode which is converted by Xilinx System Generator into HDL and synthesized in Xilinx ISE. The FSM uses a counter variable stored in a register to validate the widths and spacing between the framing pulses F1 and F2. The complete detector FSM state diagram is shown in Figure 1.8. 


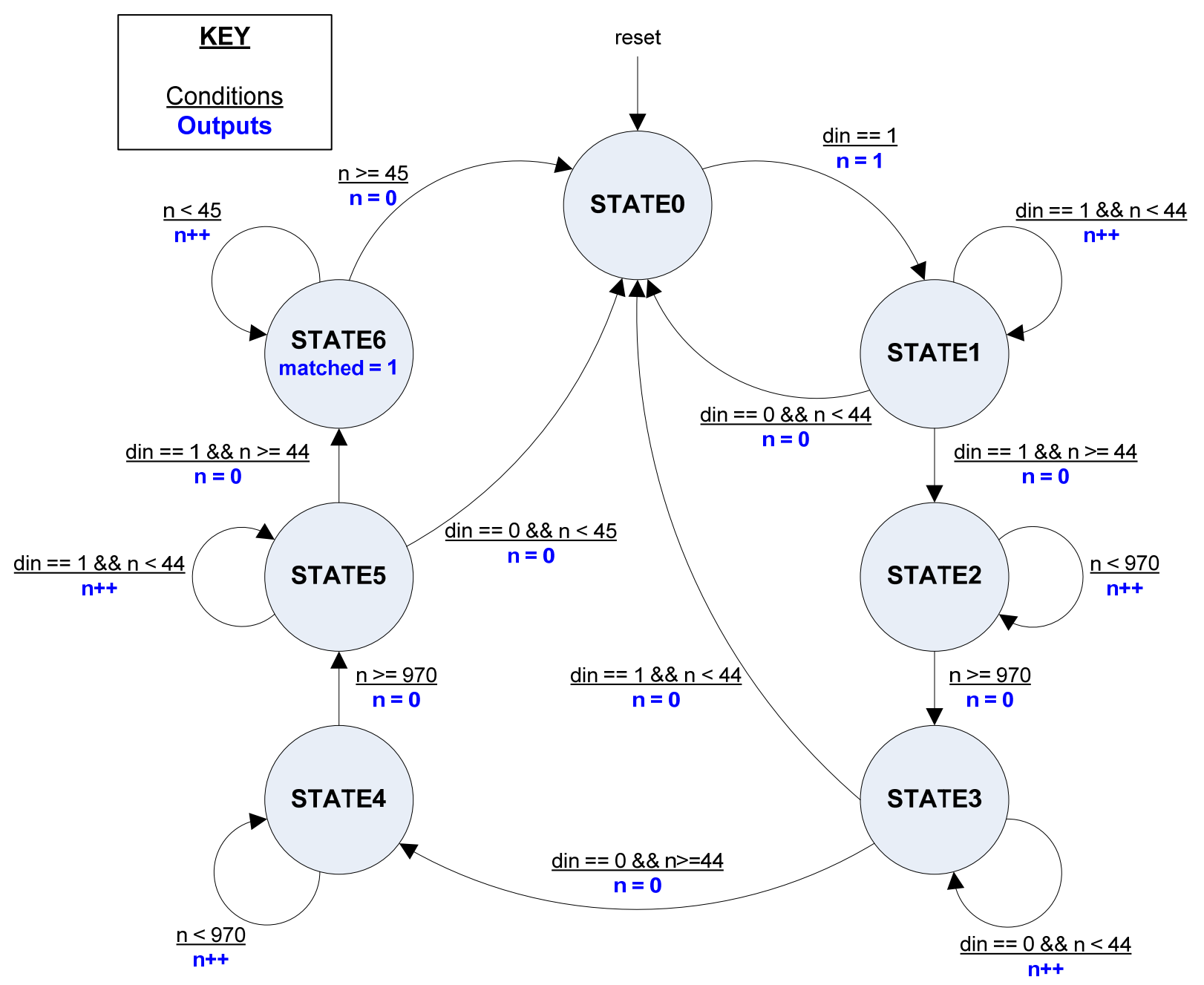

Figure 1.8: Original Detector Implementation State Diagram [18]

STATE0 waits for a rising edge on the input waveform to begin the detector FSM. STATE1 corresponds to framing pulse F1, STATE5 corresponds to framing pulse F2. STATE2 and STATE4 are placeholders for data pulse windows and STATE3 validates the empty pulse window between pulses A3 and B1. STATE6 is an output state and holds the "matched" indicator signal high after a transpond has been received and validated.

The M-code implementation of the FSM resides inside a Simulink model, shown in Figure 1.9, which also contains elements of the Xilinx System Generator software. Input and output ports are defined and mapped to physical pins or internal signals of the FPGA. A Simulink oscilloscope block coupled with test inputs imported from a 
saved MATLAB workspace provide computer simulation and testing capabilities.

The MATLAB workspace contains sampled time index and input waveform values for an IFF message.

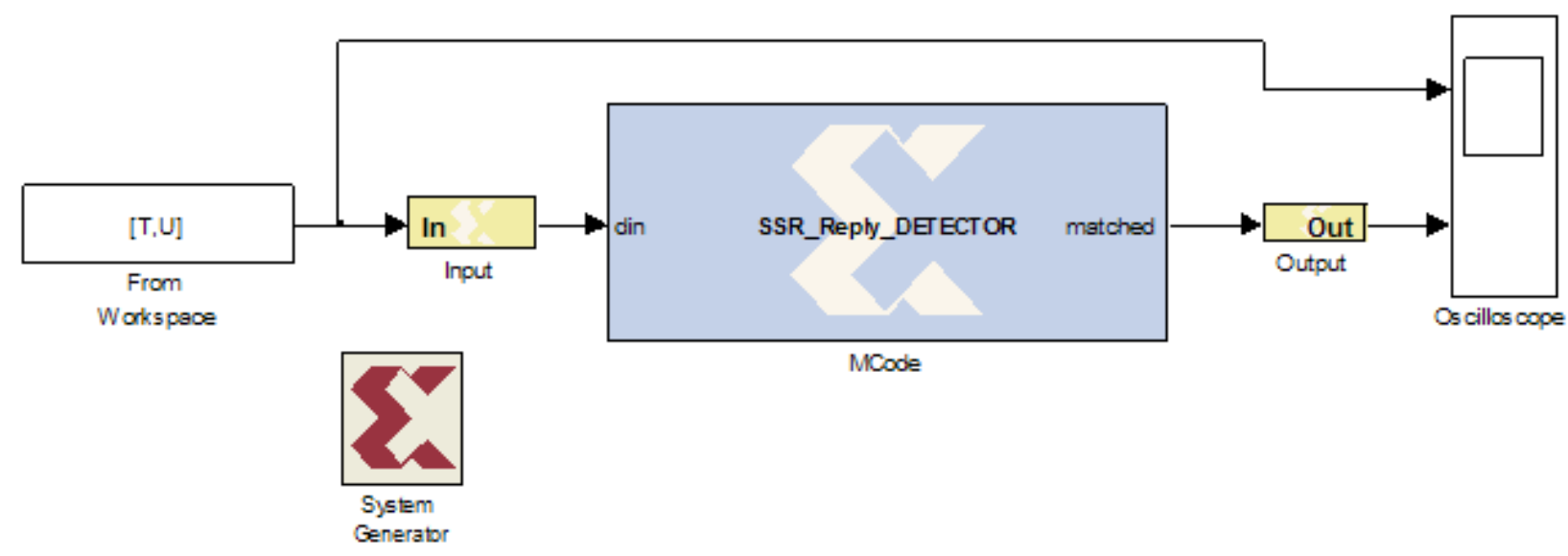

Figure 1.9: Simulink Model Containing Xilinx System Generator Elements and Detector M-Code Block [18]

The tan "Input" and "Output" blocks map signals to external ports in the HDL module, the blue "M-Code" block contains MATLAB code describing the operation of the detector finite state machine. The "From Workspace" and "Oscilloscope" blocks are used in simulation within Simulink and accept test inputs and display test outputs, respectively. The "Xilinx System Generator" block contains properties regarding simulation and HDL generation options.

The lab test setup, shown in Figure 1.10, consisted of the Xilinx ML410 development board, oscilloscope, and arbitrary waveform generator. More detail on the lab equipment is located in Section 2.4 of this report. The arbitrary waveform generator was programmed from the computer to simulate IFF Mode A/C framing pulses, while the oscilloscope was used to verify these pulses and watch for a signal from the detector hardware indicating a detected pulse. 


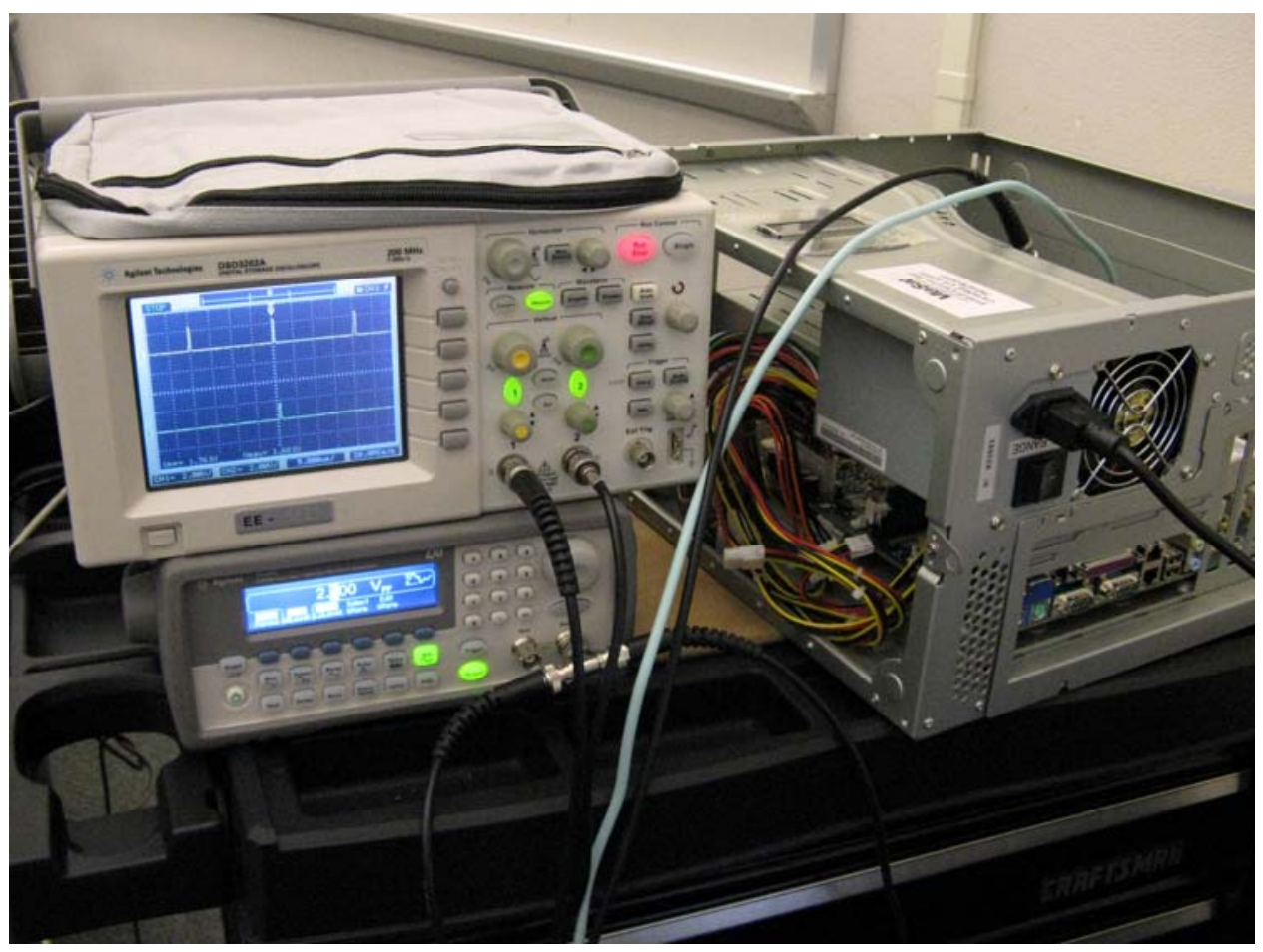

Figure 1.10: Lab Equipment and Development Platform Used by 2008 Senior Project Students [18]

(Top Left) Oscilloscope displaying IFF Mark XII Mode A/C framing pulses (Bottom Left) Agilent 33120A arbitrary waveform generator producing framing pulses (Right) Xilinx ML410 development board programmed with the detector code

The 2008 senior project students were able to successfully detect a set of IFF Mode A/C framing pulses, shown in the Figure 1.11. The original design also successfully rejects framing pulses that are not properly spaced or do not have proper pulse widths. However, the original project only looks for framing pulses and does not extract data from a message. 


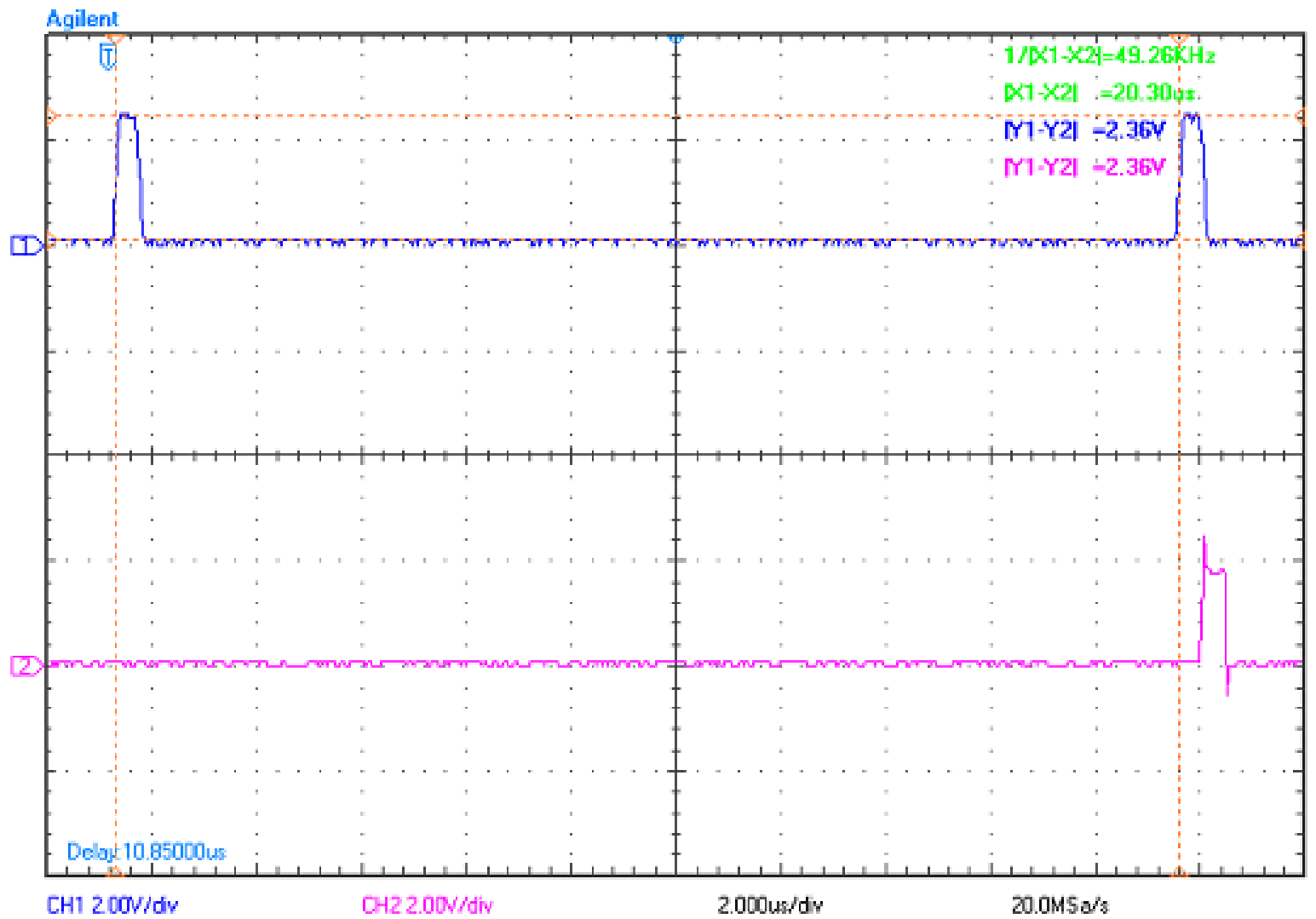

Figure 1.11: Oscilloscope Screen-Capture Showing IFF Mark XII Mode A/C Framing Pulses (Top) and a Detected Indicator Signal (Bottom) [18]

The 2008 senior project proved the viability of using Xilinx System Generator to develop IFF/SSR waveform detectors and became the foundation of this continued look at how to further abstract the design process and incorporate more features. Bland and $\mathrm{Wu}$ also obtained the software and licensing for the Xilinx software applications and the VxWorks real-time operating system developed by WindRiver, and began researching the potential use of VxWorks in a transpond detection system to interpret and send data to a user over a network. This project picks up where the 2008 project left off and investigates ways to further maximize the benefits of an abstracted design process. 


\section{DESIGN AND IMPLEMENTATION}

Based on the results of Bland and Wu's senior project, we use Xilinx System Generator again in this project to produce HDL code. However, a desire for another level of abstraction was expressed by ViaSat, and a custom MATLAB GUI was chosen as the front-end for defining the waveform a detector should be looking for. The same lab hardware (described in detail in Section 2.4) is used as the 2008 senior project and the SSR reply detector is integrated with a PowerPC microprocessor system running the VxWorks real-time operating system and custom software for interpreting message data.

\subsection{Overview}

To make the design process as easy and intuitive for the user as possible, every step in the design takes place within a graphical environment, with one exception. The exception to this is writing the embedded software to run within the VxWorks operating system: this $\mathrm{C}$ code must still be written by hand. A complete step-by-step walkthrough of the design process is available in Appendix D: User's Guide. The hierarchical overall design flow for defining and implementing a detector is shown in Figure 2.1. 


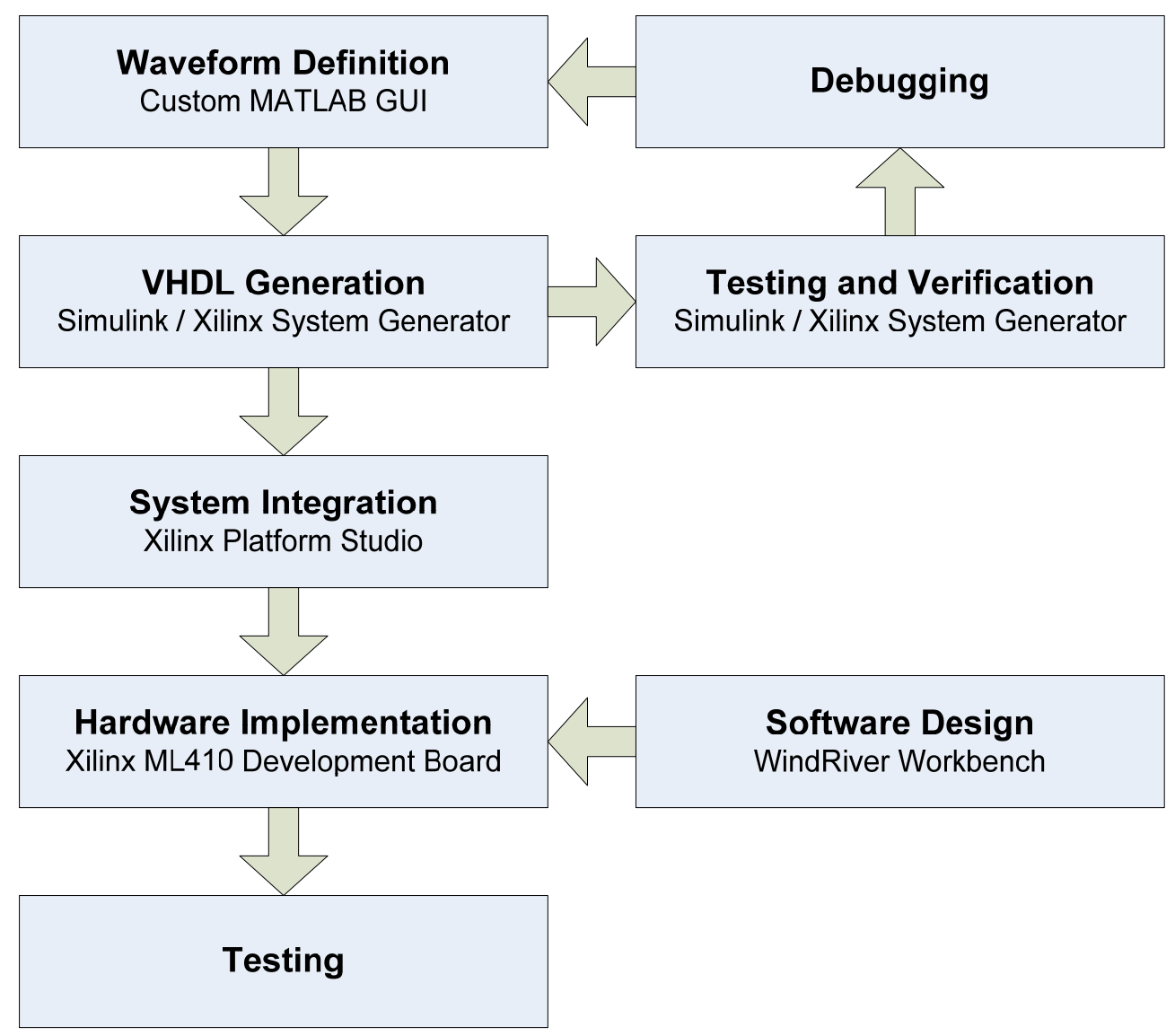

Figure 2.1: Overview of the Detector Design and Implementation Process

First, a detector is defined in a custom MATLAB GUI and exported to MATLAB M-code to be used by Xilinx System Generator. A Simulink model with Xilinx System Generator elements contains this detector's M-code and supporting peripherals. Testing can be done in System Generator with simulation software and test inputs saved in MATLAB variables. Xilinx System Generator converts this Simulink model into HDL code which is integrated, along with the VxWorks operating system, into a system hardware design in Xilinx Platform Studio. The final implementation is on the Xilinx ML410 development board and custom software is downloaded to the VxWorks system to perform additional data processing. All elements of this design flow take place within graphical environments, with the exception of the software design in WindRiver Workbench.

The first step in designing an IFF/SSR reply detector is to define the waveform shape in a custom MATLAB GUI. The user inputs timing parameters that define the pulses present in a message and their corresponding meanings (framing pulses, data pulses, etc.). Testing and verification of the detector algorithm can be performed from 
within the Simulink and Xilinx System Generator environment, HDL code is also generated by Xilinx System Generator from the waveform specifications. System integration takes place in Xilinx Platform Studio where the reply detector is imported as a custom peripheral and connected to supporting hardware and the PowerPC processor. Xilinx Platform Studio then converts the design to a hardware image that can be downloaded to the development board. Embedded software to be used in the VxWorks operating system is written in the WindRiver Workbench suite and downloaded to the device. This software in the VxWorks environment performs additional processing on the data extracted from transponds detected by the custom hardware.

\subsection{Front-End GUI for Waveform Definition}

A front-end GUI runs within MATLAB and allows the user to define waveforms and indicate which pulses contain data (and the corresponding modulation schemes) and which pulses are framing pulses. With multiple possible waveforms the detector could be looking for, a means of saving and loading waveforms became a priority. Save files are stored in *.iff file formats (a different extension on top of a MATLAB

*.mat file) and can be assigned and restored from within the front-end GUI. Figure 2.2 shows an IFF Mark XII Mode S waveform that could be entered into the GUI. 


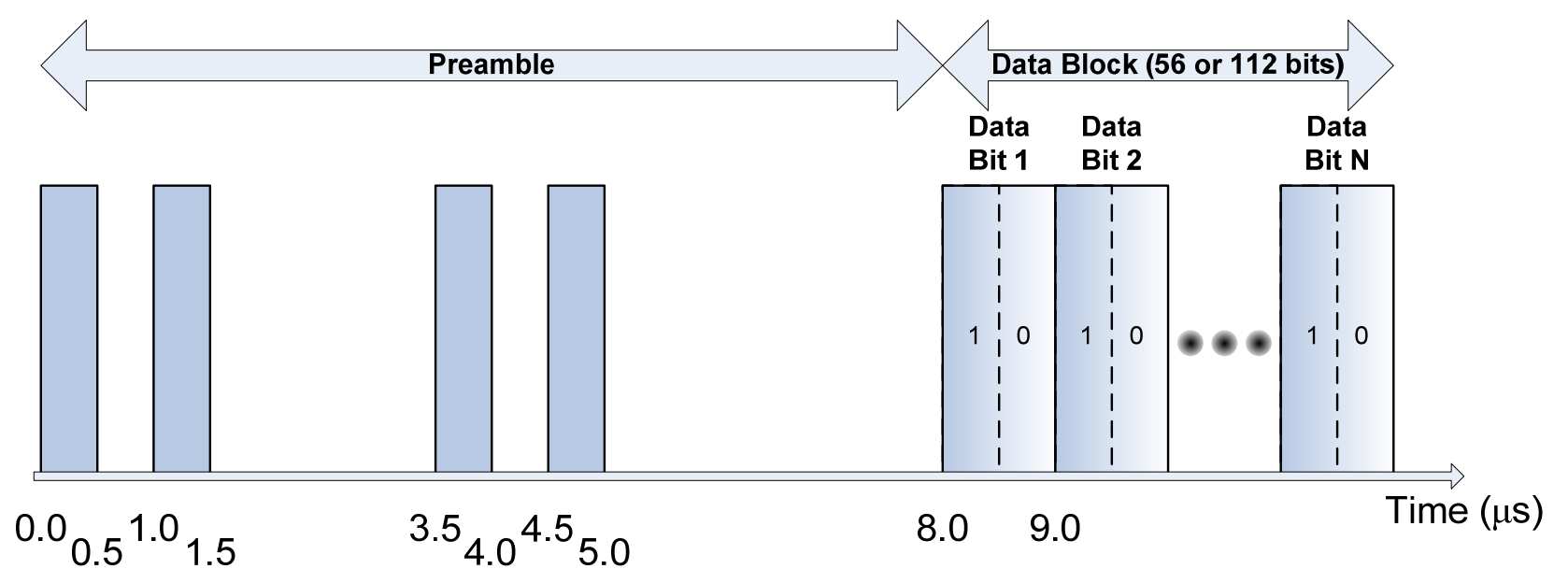

Figure 2.2: IFF Mark XII Mode S Waveform, Shown as an Example of What Can be Defined and Saved by the Waveform Definition GUI [11]

The first four pulses, known as the preamble, are framing pulses which do not contain data but are required for a properly formatted message. The following 56 or 112 pulses contain data in a pulse position modulated scheme: if the pulse is present in the left half of the pulse window the data bit is a 1, if the pulse is present in the right half of the pulse window the data bit is a 0. Each pulse is $0.5 \mu$ side, with corresponding pulse windows for the data bits at $1.0 \mu$ s wide.

From within the GUI, users can define individual pulse locations, widths, and tolerances as part of a larger waveform. Pulses can be classified as either framing pulses or data pulses with a variety of modulation schemes. Framing pulses contain no modulated data, but are required for a message to be considered valid. Data pulse modulation schemes include Pulse Position Modulation (PPM) and On-Off Keying (OOK). Each pulse can also be assigned a percentage tolerance that will be used to validate a received message. After correctly setting up a desired waveform, the user presses the "Export to Simulink / Xilinx System Generator..." button in the "Export" menu. This process automatically creates an M-code (*.m) file with a finite state machine tailored to detecting the given waveform. Figure 2.3 shows the same IFF Mark XII Mode S waveform as in Figure 2.2, after it is entered into the custom GUI and ready for export into Xilinx System Generator M-code. 


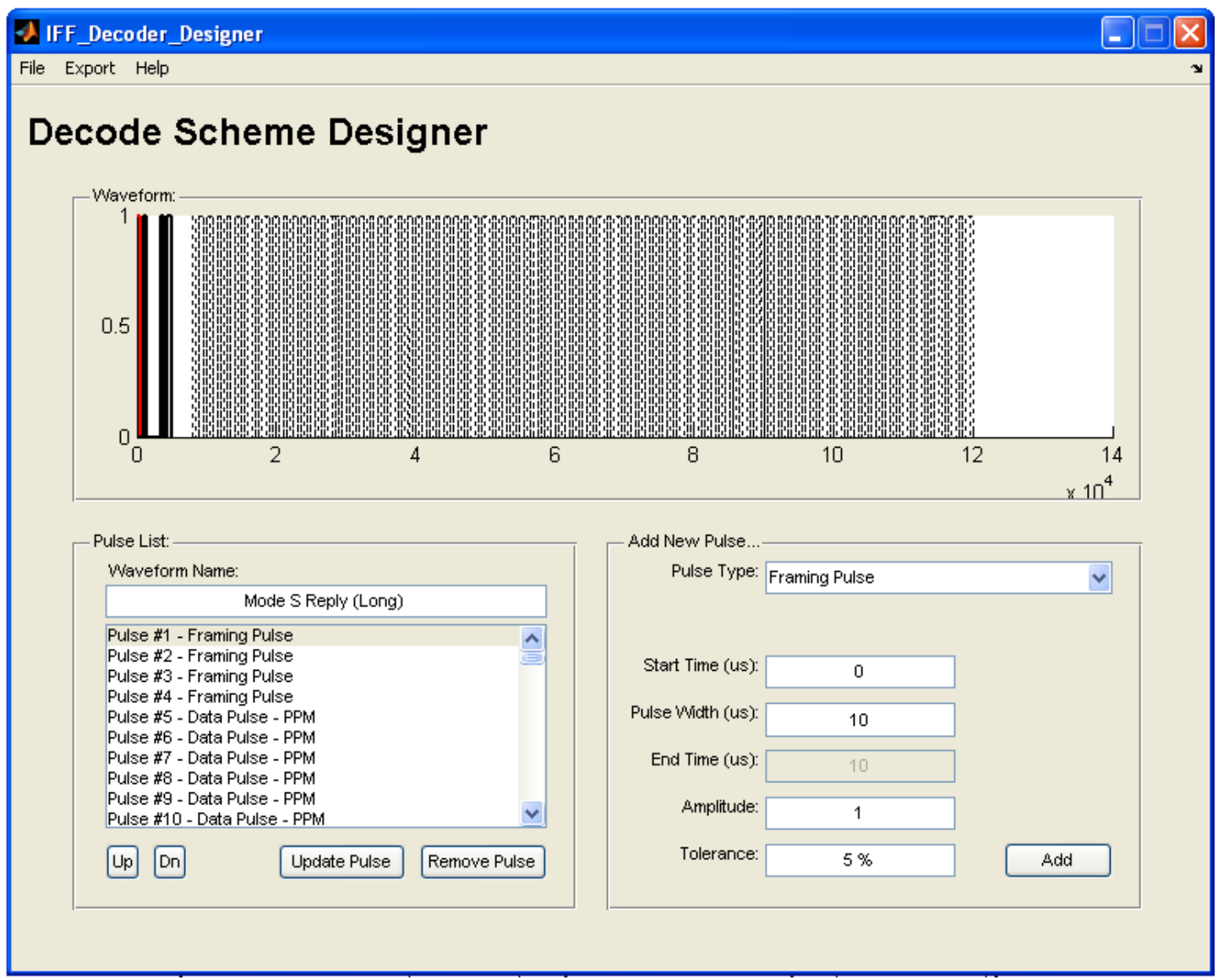

Figure 2.3: The Custom Waveform Definition GUI

The waveform currently loaded is an IFF Mark XII Mode S long transpond. The dotted pulses contain data in pulse position modulation while bold pulses are framing pulses. The GUI elements in the "Add New Pulse..." frame allow the user to adjust pulse parameters and add additional pulses to the currently loaded waveform. The listbox in the "Pulse List" frame lists every pulse in the currently loaded waveform and allows the user to delete, update, or move pulses up and down in the list. The currently selected pulse is colored red in the waveform display. The "Export" menu contains the button to export the detector to M-code suitable for use in the Simulink model with Xilinx System Generator.

The script that generates M-code for the finite state machine is defined in "IFF_Decoder_Designer_Export.m" and utilizes a building-block approach. The GUI passes the export script a structure containing information about each pulse in the waveform including pulse type, modulation scheme, start time, pulse width, and 
tolerance. Each type of pulse has a prewritten framework with timing parameters waiting to be plugged in to define the pulse width and start time. The export script strings together these building blocks to form a complete detector FSM, tagging on input and output states at the beginning and end, respectively. The following figures outline the flow diagram of the generated FSM.

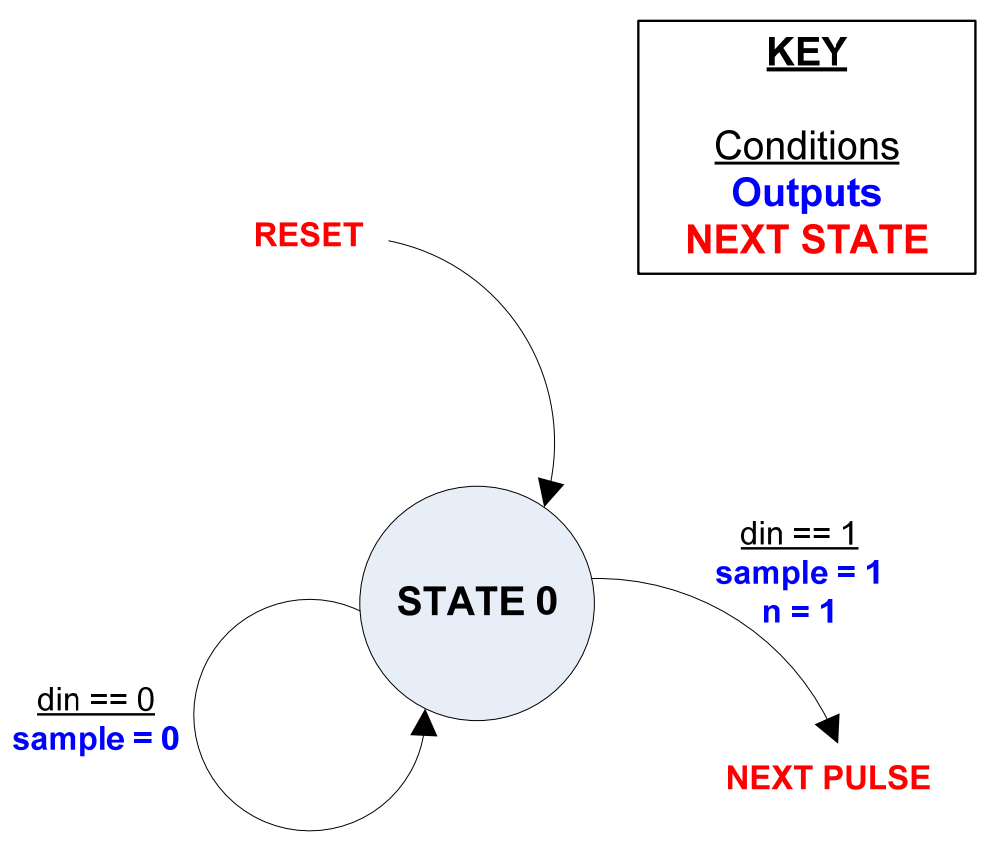

Figure 2.4: State 0, Waiting for the First Rising Edge of the Frame to Trigger the Detector FSM

The first state of the FSM, state 0 is common to all detectors created by the GUIbased waveform definition program. The state transition diagram associated with state 0 is shown in Figure 2.4. This state simply waits for the first rising edge of a message frame to trigger the rest of the FSM operation. When reset, the FSM reverts to state 0 . 


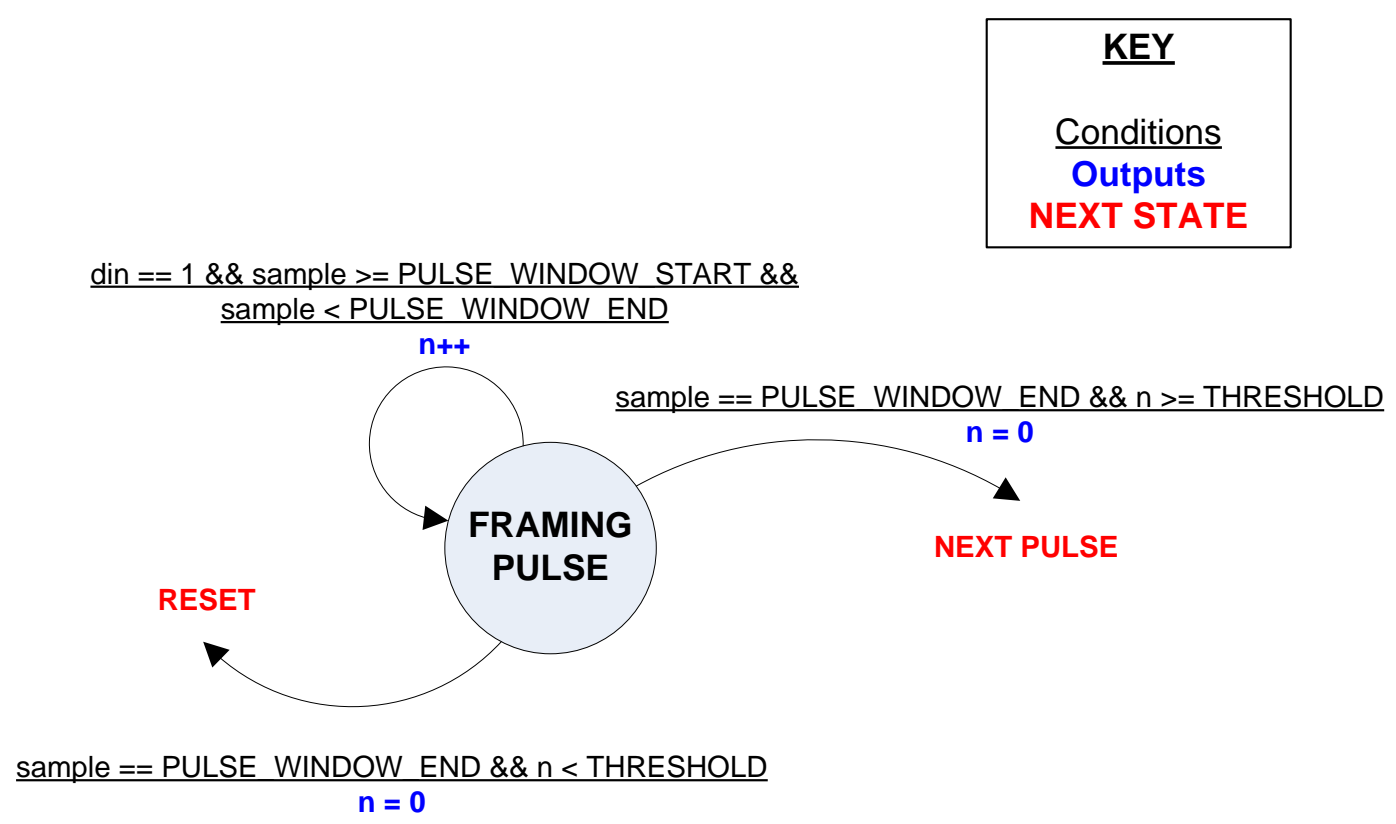

Figure 2.5: Framing Pulse Detection Block Flowchart

A timer counts the number of high samples during the framing pulse window. This value is compared to a threshold to validate the framing pulse. The FSM moves to the next state if the pulse is valid, or resets if the pulse is invalid.

Framing pulse detection blocks will send the FSM to the next state if the pulse is correctly formed, or send the FSM back to state 0 if a pulse is incorrectly formed. The state transition diagram corresponding to a framing pulse is shown in Figure 2.5.

Framing pulses contain no data but are defined as required for a message to be valid. The detector block uses a timer to count the number of high input samples during the framing pulse window. This number is compared against a threshold value to check validity. Depending on the user's requirements, a framing pulse could be required to be continuous or just summed over the window to compare against the threshold value. 

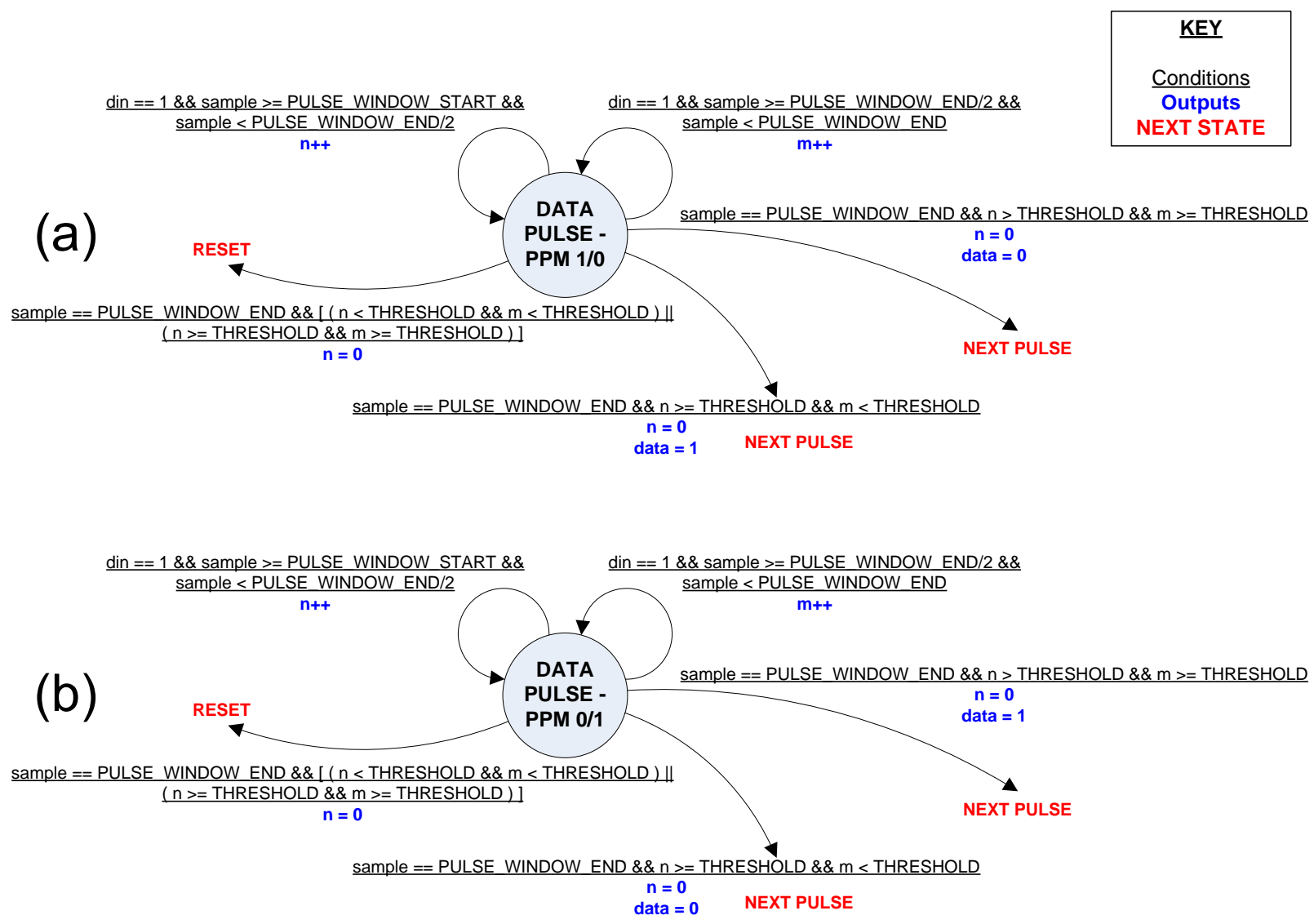

Figure 2.6: Pulse-Position Modulated (PPM) Data Pulse Block Flowcharts

The PPM data detection states demodulate data and if a pulse is properly formatted, send the FSM to the next pulse state. If invalid, the FSM resets.

(a) In PPM 1/0, a pulse in the first half of the window corresponds to a data 1.

(b) In PPM 0/1, a pulse in the first half of the window corresponds to a data 0.

Pulse-position modulated (PPM) data pulse blocks, diagrammed in Figure 2.6, utilize two timers. The first timer counts the number of high input samples in the first half of the pulse window, and the second timer counts the number of high input samples in the second half of the window. These counter values are then compared against a threshold to determine if the encoded data is a 0,1 , or invalid. In "PPM $1 / 0$ " the first half of the window represents a data 1 and the second half represents a data 0 , and vice versa for "PPM 0/1". A data pulse is only valid if exactly one threshold is 
reached (a data pulse cannot simultaneously be 0 and 1). Invalid pulses will reset the FSM. The data value that is demodulated from the detected message pulse is written into a temporary data register, which will be rewritten into a FIFO memory block if the entire message frame is deemed valid. Note that the FIFO writing scheme described here is the desired one and a workaround is described later that circumvents a bug in the Xilinx software.

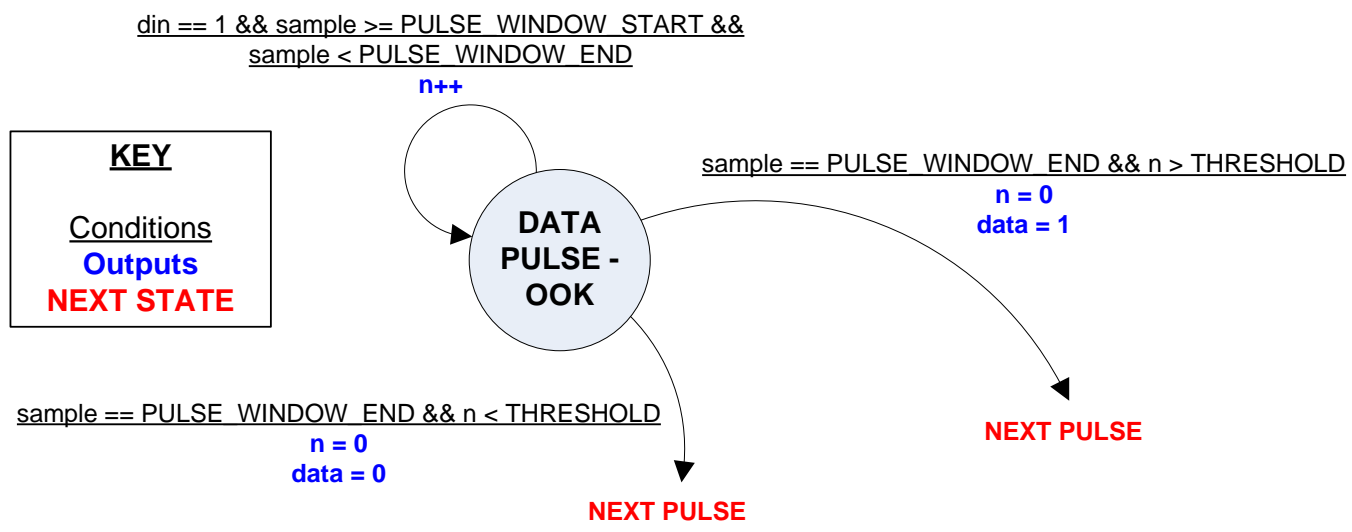

Figure 2.7: On-Off Keyed (OOK) Data Pulse Block Flowchart

If a pulse is present and wide enough, the OOK detector block writes a 1 of data. If not, the OOK detector writes a 0 of data. The FSM then moves to the next state.

On-off keyed (OOK) data pulse blocks, shown in Figure 2.7, look for the presence of a correctly formed pulse to indicate a data 1 and the absence of such a pulse to indicate a data 0 . The detection works just like with the framing bits: a counter is used to compare the number of high input samples during the pulse window and this number is compared to a threshold. Again, according to the user's requirements, an OOK data pulse could be required to be contiguous rather than just summed over the pulse window. The data value is written to the temporary data register and will be 
written to the data FIFO when the entire message is deemed valid. Note also that the FIFO writing scheme described here is the desired one and a workaround is described later in this section that circumvents a bug in the Xilinx software.

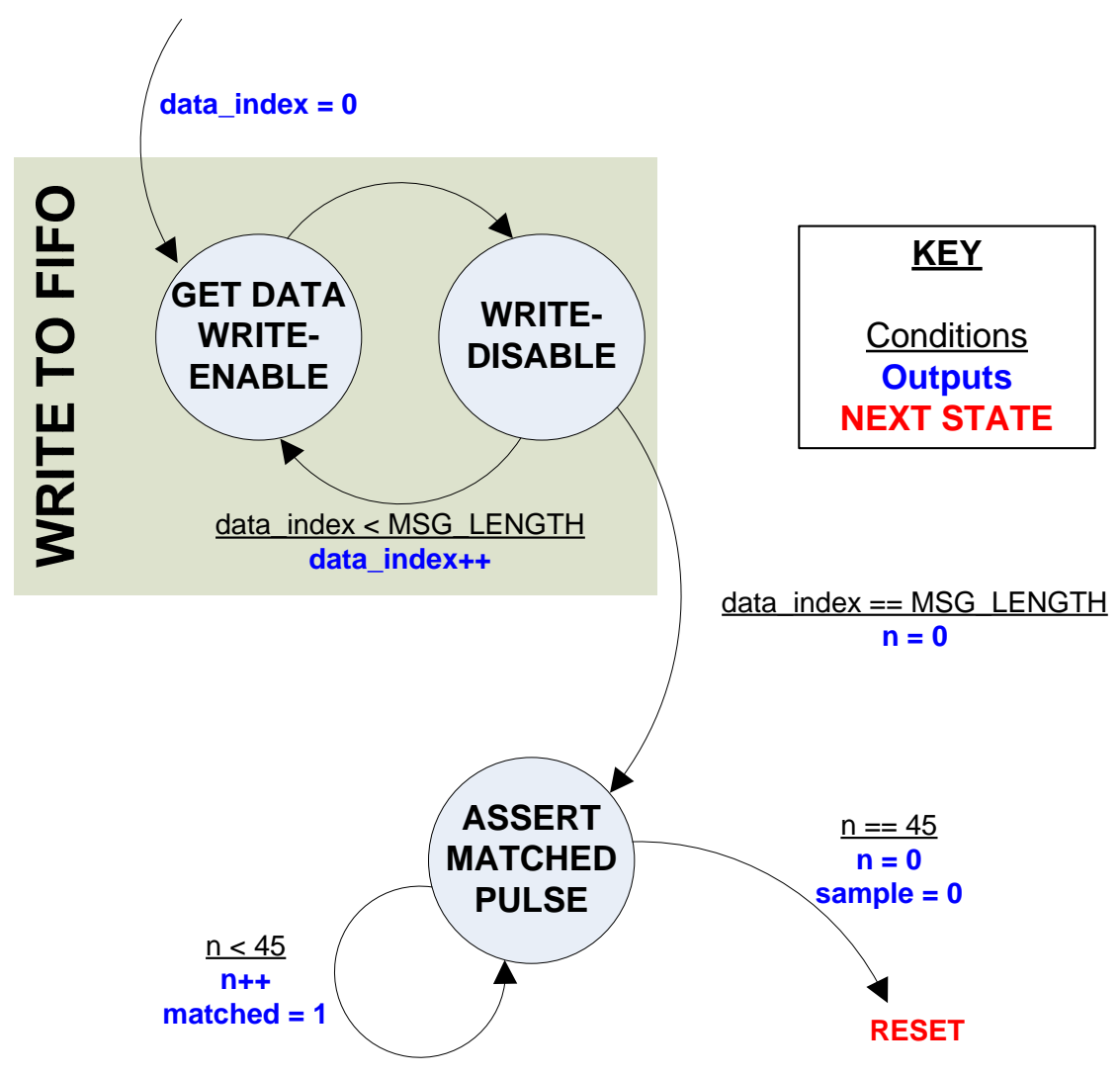

Figure 2.8: Desired Output Stage Flowchart, Common to All Detector Implementations The output state writes data from the temporary register to the FIFO then asserts the "matched" indicator signal. Afterwards, the FSM resets to look for a new incoming transpond.

All detectors created by the GUI-based waveform definition program use a common output stage of the FSM, the desired version of which is shown in Figure 2.8. The message must be properly formed in order for the FSM to make it to these states without being reset. The output stage should first write the data from the 
temporary data register into the FIFO. Then, an output signal called "matched" is held to logic high for 45 samples. This matched signal is routed to an external pin on the FPGA so operation of the FSM can be assessed on the oscilloscope.

Writing the data from the temporary data register to the FIFO in the output stage is desirable because only data from a well-formed IFF packet will be written. Unfortunately, due to some bugs within the Xilinx System Generator software, this was not possible. Extensive experimentation revealed problems with indexing a memory array in the HDL code produced by System Generator and reinterpreted by Xilinx Platform Studio. When trying to access an indexed value of a memory array, either an error is thrown by System Generator, or only the first item of the array is properly indexed. It is unknown whether these bugs reside in the conversion from Mcode to HDL, from HDL to a custom peripheral in the Xilinx EDK, or both. In simulation within Simulink and the Xilinx System Generator framework, the indexing and FIFO writing worked as expected, but the implementation in hardware following synthesis did not match the simulation. 


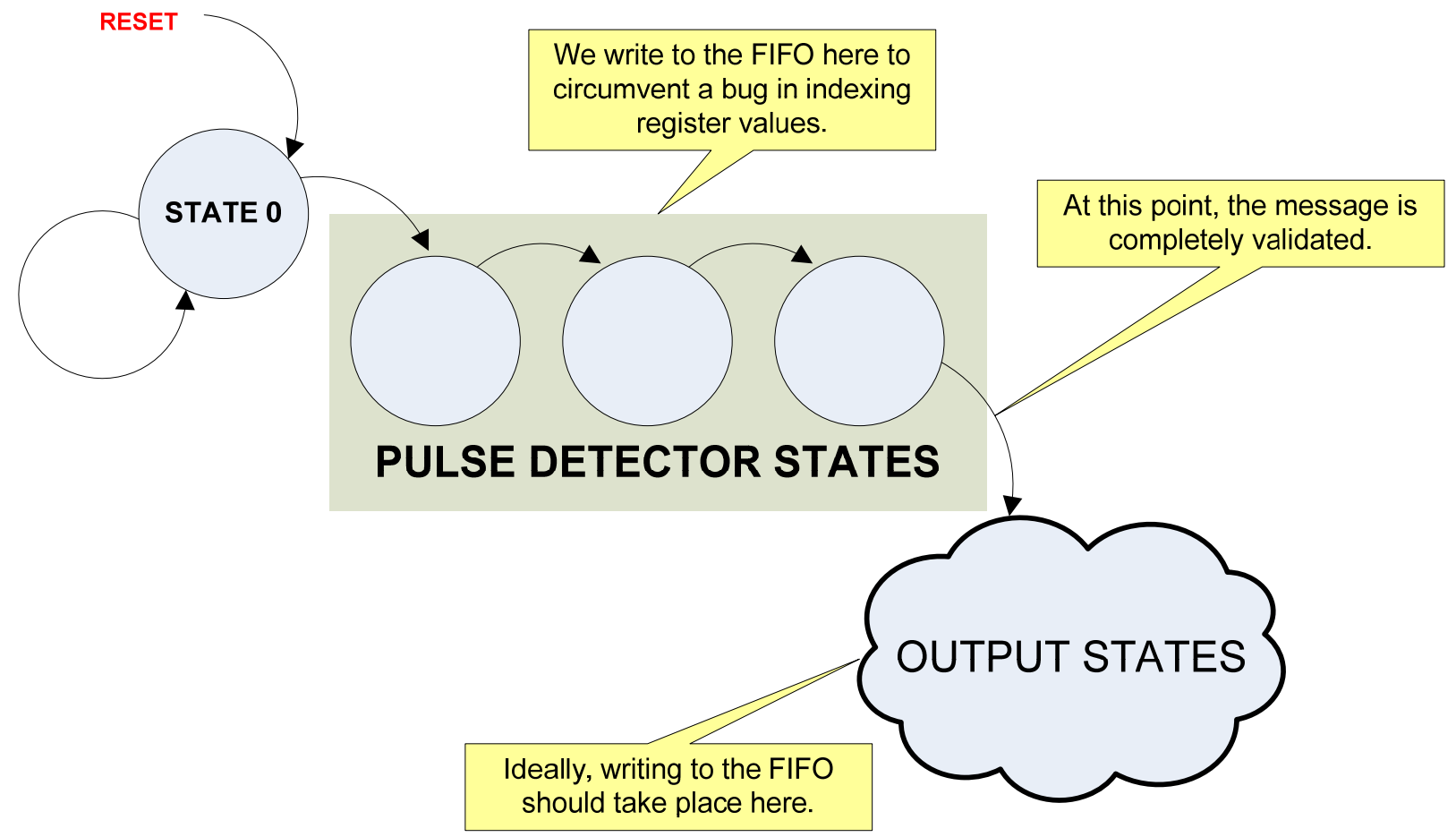

Figure 2.9: Writing to the FIFO During Pulse Detection Is Not Ideal, But Circumvents a Bug Ideally, the detector should write to the FIFO after all pulses have been validated. However, a bug with indexing data registers prevents this implementation. Instead, data is written to the FIFO during the pulse detection states.

As an alternative for use in the proof-of-concept design, as soon as a data bit is interpreted in its corresponding FSM state, the data is written directly to the FIFO bypassing the temporary data register altogether. The workaround for writing to the FIFO is shown in Figure 2.9. This fix allows data to be correctly written to the FIFO, but in the event of a message falling out of tolerance after several bits of data are written, some garbage data will be written to the FIFO. Writing garbage data to the FIFO can also contribute to problems with the software falling out of sync while reading data. For this detection system to move past proof-of-concept and into production, this indexing problem is a major issue that needs to be addressed, either by Xilinx fixing the indexing bug or a user employing a clever workaround. 


\subsection{VHDL Generation}

The Simulink model used by Xilinx System Generator to create VHDL code is based off the original design in Bland and Wu's senior project. However, several additions have been made to the overall schematic, shown in Figure 2.10. A FIFO memory block now provides storage of demodulated message data. The FIFO also requires corresponding input and output ports for interfacing with the other parts of the system. Between the FIFO read-enable ("fifo_re") input signal and the FIFO itself, an edge trigger (pictured in Figure 2.10c) guards against the possibility of longer-than-ideal read signals from the processor draining the entire FIFO of data. Additional test points are also in place for debugging the FIFO signals. Based on a suggestion in the System Generator User's Guide, delay blocks surround the M-code critical path. These delay blocks are supposed to help the synthesizer meet minimum timing requirements. 


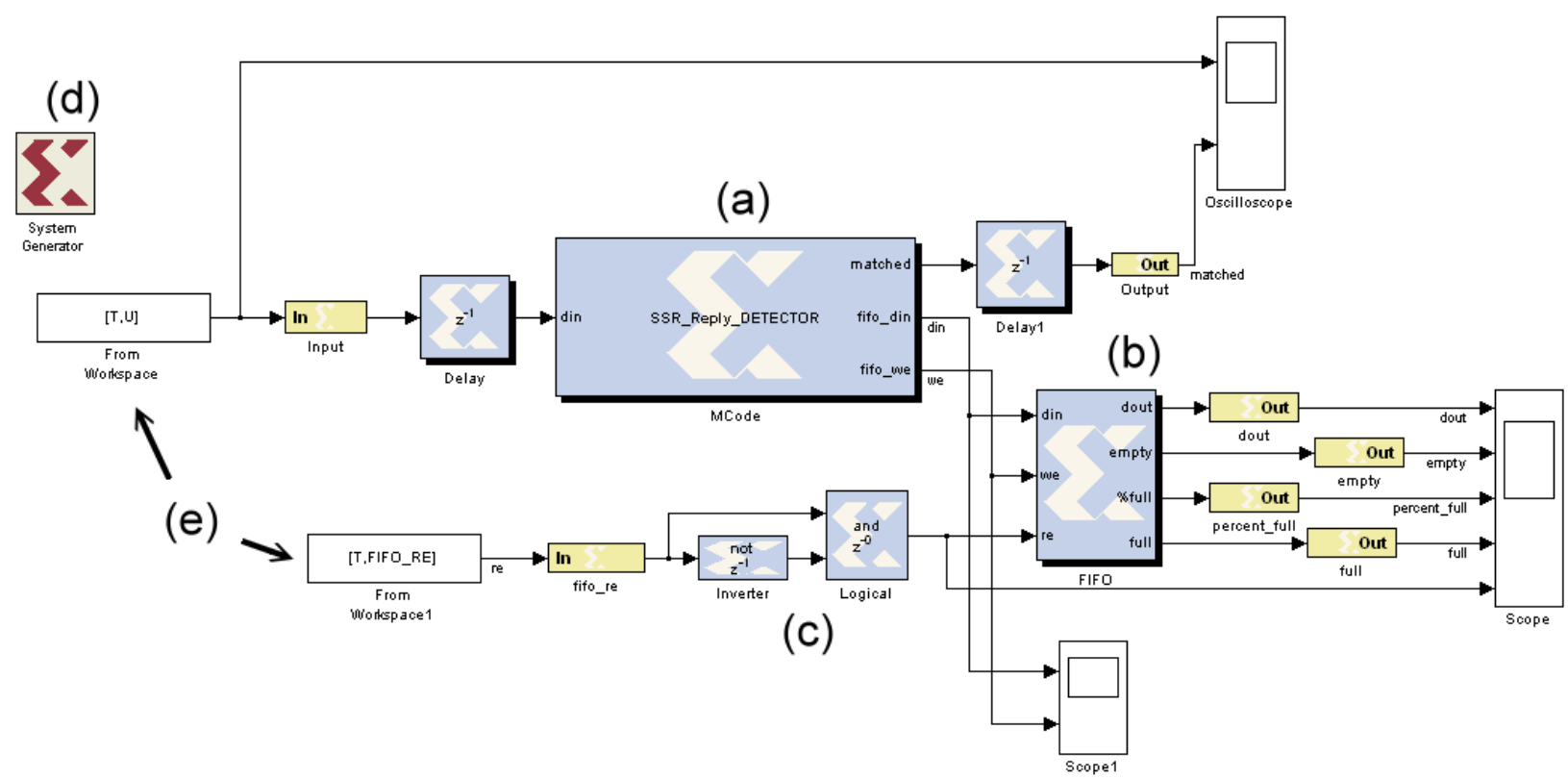

Figure 2.10: Simulink Model Used by Xilinx System Generator to Perform Testing and Write VHDL Code

(a) The M-Code block contains the detector finite state machine code generated by the GUI export function. This FSM also controls writing data to the FIFO and manages the "matched" output signal.

(b) The first-in, first-out (FIFO) memory block stores data from detected messages.

(c) An edge trigger ensures only one value is read out of the FIFO at a time when polled by the software running in the VxWorks system.

(d) The Xilinx System Generator block contains HDL generation options such as the target device, output file name, and optimizations.

(e) The "From Workspace" blocks provide test input vectors from MATLAB variables for use in simulation within the Simulink and Xilinx System Generator environment.

(Not Lettered) The tan input and output ports indicate to the Xilinx software which signals are to be connected externally to the generated HDL module.

The M-code block still contains the detector FSM, but instead of being handwritten, this code is generated by the GUI's export function. Figure 2.11 shows the step-by-step process of converting the graphical representation of the detector to Mcode to HDL. Once the waveform of interest has been defined in the custom GUI and exported to the M-code block in the Simulink model, Xilinx System Generator takes approximately 5 minutes to convert the design into VHDL code. System Generator 
converts the Simulink model to HDL by combining predefined modules from the Xilinx Blockset library with M-Code interpretation and connection and routing logic.

When the design is converted to HDL these black box modules are implemented in *.ngo, *.ngc, and *.edf files.

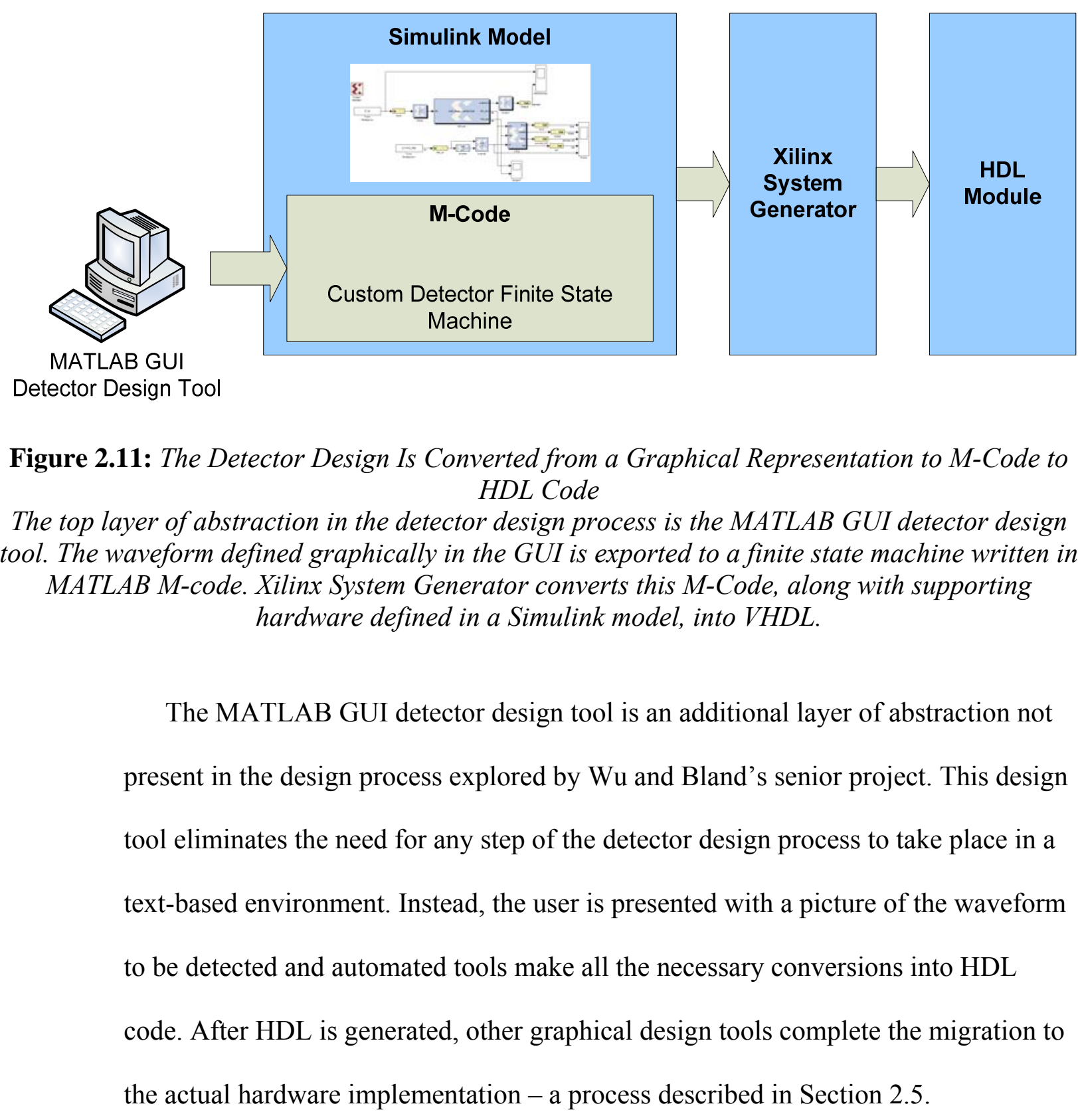




\subsection{Hardware Platform}

ViaSat provided Cal Poly with a Xilinx ML410 development board for this project. The ML410 board contains a Xilinx FX60 Virtex-4 FPGA with an on-chip PPC405 PowerPC processor. The ML410 board also provides supporting hardware for the FPGA such as Ethernet MAC and PHY layers, onboard memory, serial and parallel interfaces, and a power supply. An overview of the ML410 board's subsystems is shown in Figure 2.12. The ML410 board is programmed over a JTAG interface; the computer connects to the JTAG cable with the parallel port and powers the cable with a PS/2 connection. The computer used in this project lacks a PS/2 connector on the motherboard, so instead a USB to PS/2 adapter powers the JTAG cable. 


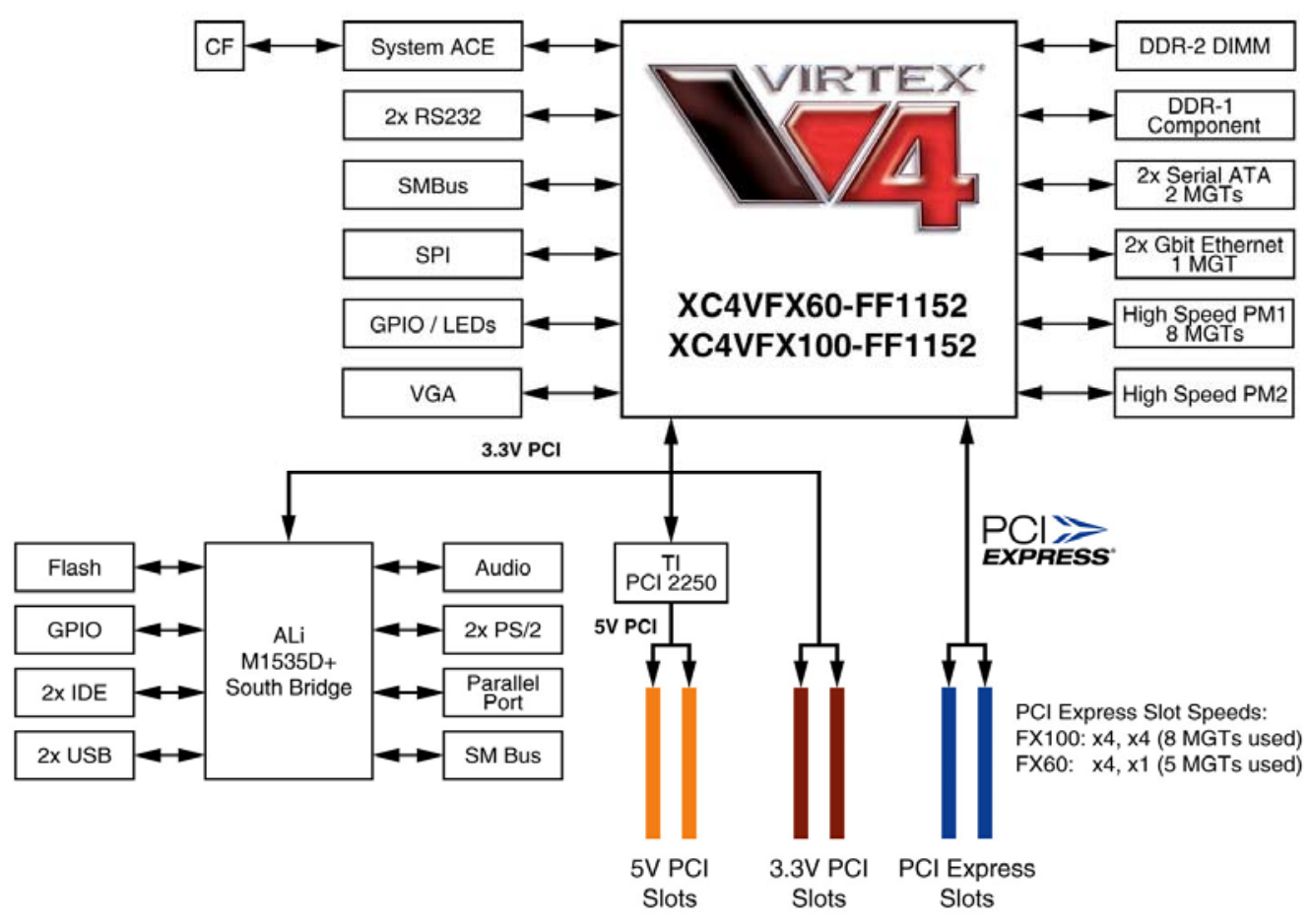

Figure 2.12: Block Diagram of the ML410 Development Board's Subsystems [19]

The heart of the ML410 development is the Xilinx FX60 Virtex-4 FPGA. The FPGA fabric houses our custom detector hardware. The FX60 chip also contains a PPC405 PowerPC processor which we use to run the VxWorks real-time operating system and custom software used for additional data processing. Also shown are I/O elements of the ML410 including Ethernet and serial.

The ML410 board is extremely full-featured and includes many subsystems beyond the requirements of the IFF detector implementation explored in this project. However, the complexity of the board also presented a challenge in getting direct access to the pins of the FPGA. Most onboard input and output pins interface to the FPGA through some type of supporting hardware, whereas we wanted a direct connection to a pin on-chip. To solve this, we interfaced the input and output signals to the FPGA through two SMA connectors (J17 and J36) on the ML410 board designed for high-speed off-chip clock signals. The SMA connectors used for input 
and output are shown in Figure 2.13. This direct interface between the SMA

connectors and the FPGA was first explored in the 2008 senior project by Bland and $\mathrm{Wu}[18]$.

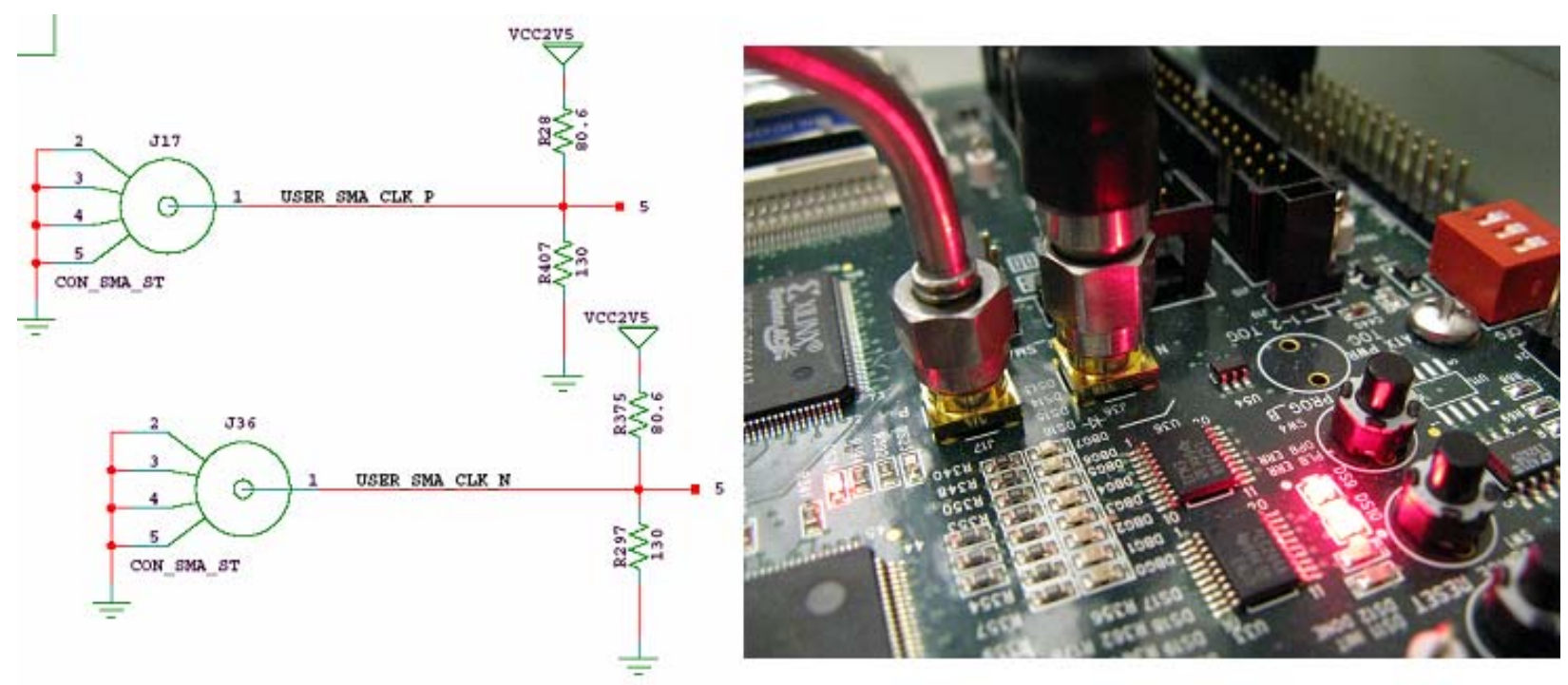

Figure 2.13: Schematic Diagram and Photograph of the Two SMA High Speed Clock Interface Ports [18]

These SMA ports provide direct access to pins J17 and J36 on the Xilinx FX60 Virtex-4 FPGA. $J 17$ serves as the input pin and is connected directly to the arbitrary waveform generator. J36 is wired to the "matched" signal and provides indication when a message is detected.

As in the 2008 senior project, test inputs are defined on the computer and generated and sent to the device via the arbitrary waveform generator. Test waveforms are saved as comma-separated value (*.csv) files and loaded to the arbitrary waveform generator with Agilent software. Our particular arbitrary waveform generator, the Agilent 33120A, is capable of handling waveforms of up to 65535 sampled data points. The device should be set to 2.1 volts peak-to-peak amplitude and -0.7 volts DC offset to get the logic levels within spec at the input 
terminal to the ML410. Operating frequency can be calculated as in the following example, Equation 2.1:

\section{$0.01 \mu \mathrm{sec} /$ sample $* 65535 \mathrm{samples} /$ message $=655.36 \mu \mathrm{sec} / \mathrm{message}$ $1 / 655.36 \mu \mathrm{sec} /$ message $=\mathbf{1 . 5 2 5 8 7 8 9 1} \mathbf{~ k H z}$}

Equation 2.1: Calculating the Arbitrary Waveform Generator Operating Frequency

An oscilloscope and a PC are used to verify the correct operation of the detector system. Our PC is used for both design of the detector in MATLAB, Simulink, and Xilinx System Generator, development of the embedded software in WindRiver Workbench and for verification of correct detector operation in Wireshark. The oscilloscope, an HP 54501A, displays both the simulated IFF transpond signals generated by the arbitrary waveform generator and the "matched" signal controlled by the detector finite state machine. When a "matched" pulse is observed on the oscilloscope, a transpond has been correctly detected. Software running in the VxWorks operating system on the PowerPC processor sends UDP data packets to a host computer when messages have been decoded, interpreted, and formatted. Further description of the software system is in Section 2.6 of this report.

\subsection{System Integration in Xilinx Platform Studio}

System integration is accomplished by importing the VHDL code generated by Xilinx System Generator as a custom peripheral into a Xilinx Platform Studio project. The base project itself is a board support package (BSP) designed specifically for the 
hardware platform to interface with the VxWorks real-time operating system. The BSP contains compatible hardware devices as well as the corresponding software drivers for VxWorks to function correctly. The Xilinx Platform Studio environment is shown in Figure 2.14.

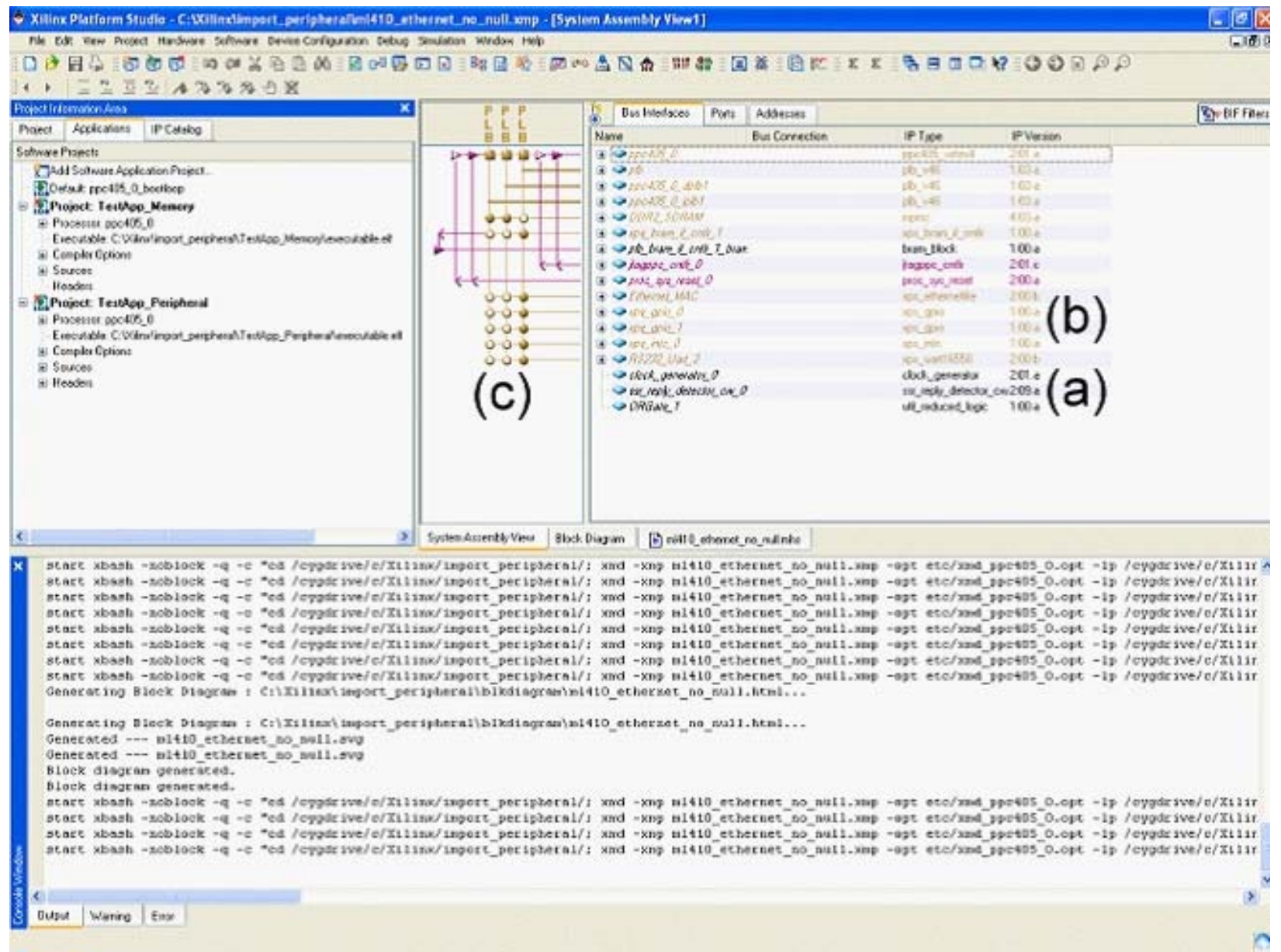

Figure 2.14: System Integration Takes Place within the Xilinx Platform Studio Environment

(a) The custom transpond detector imported as a peripheral device.

(b) Two GPIO blocks interface the detector peripheral with the Processor Local Bus (PLB).

(c) The bus connection pane shows the connections between system components.

The main interface between the PowerPC processor (running VxWorks) and the other system peripherals is the Processor Local Bus (PLB). Devices connected 
directly to the PLB must have compatible input and output ports and follow the data flow scheme defined in the PLB standard. Figure 2.15 shows the overview of the PLB bus architecture.

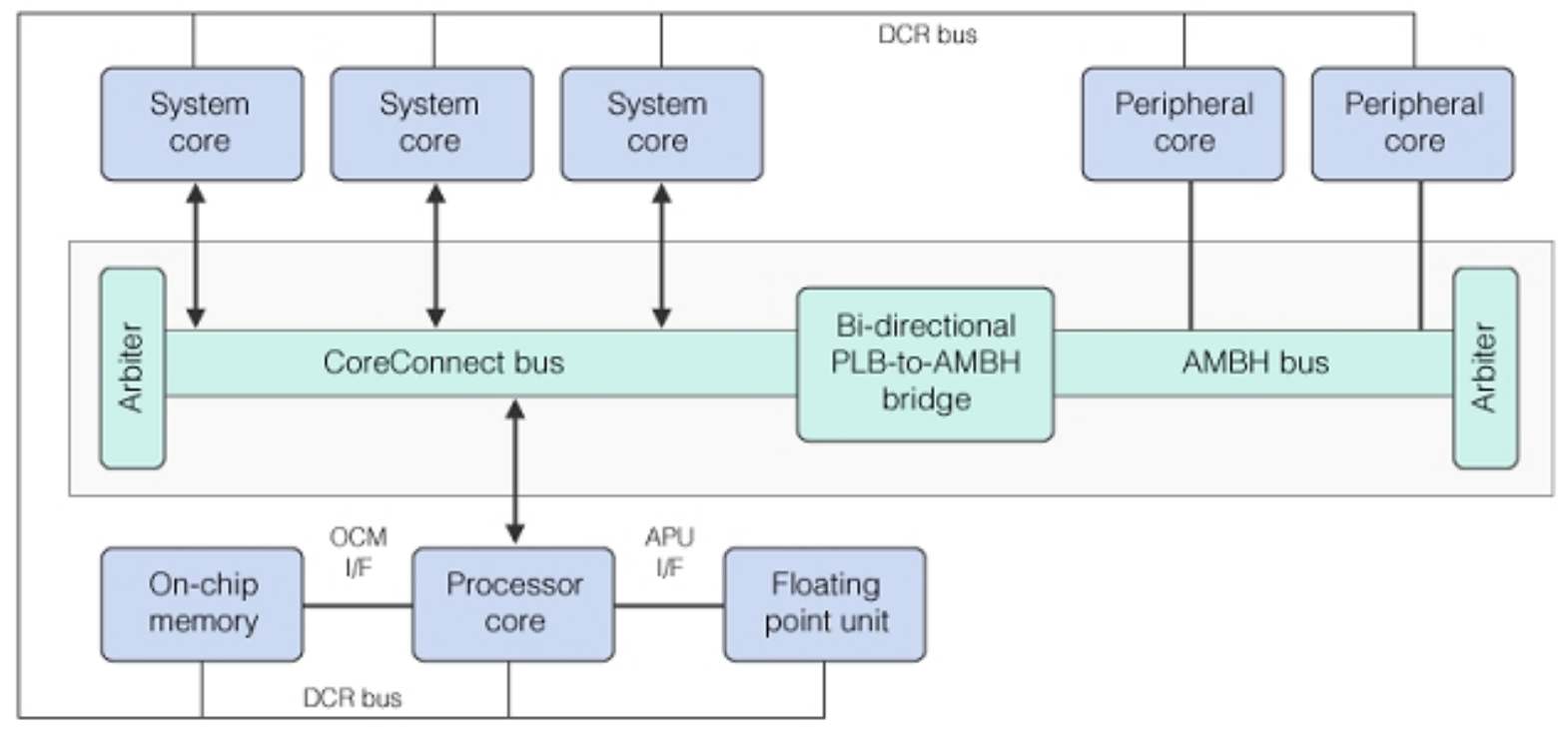

Figure 2.15: The PLB Bus Architecture [20]

The PLB connects the processor core, supporting system cores, and peripheral hardware. In this project, a GPIO core interfaces the PLB with our custom detector peripheral.

Instead of adapting our custom detector peripheral to work directly with the PLB, we chose to use a General Purpose Input/Output (GPIO) IP core as the interface between the PLB and our custom peripheral. The GPIOs are shown in context with the other system components in Figure 2.14. Each GPIO device is capable of a maximum width of 32 bits per channel with a maximum of two channels. We actually use two GPIOs to interface with the detector peripheral: one GPIO handles the FIFO read-enable input signal ("fifo_re"), the other is dual-channel and handles the FIFO empty flag and the FIFO output data. 
Converting the System Generator VHDL code into a custom peripheral is easiest by using the Import Peripheral Wizard under the Hardware menu in Xilinx Platform Studio. After selecting the generated ISE project file (*.prj) and all black-box cores for the FIFO and other elements, uncheck all bus options and skip any remaining questions. After running the Import Peripheral Wizard, the custom detector peripheral appears in the connections window with ports for clock, FIFO read-enable, FIFO data out, FIFO empty flag, the "matched" flag, data in, etc. The device can then be manually attached to the external pins and GPIO interfaces in the connections window by using the drop down menus for each signal. The constraints file also was modified to route signals to the external pins and contains the actual pin reference designators corresponding to each signal (e.g. J16). Users wishing for only the input waveform and "matched" flag to be routed to external pins should not have to modify the constraints file.

\subsection{Embedded Software}

The WindRiver VxWorks real-time operating system is an industry standard operating system for real-time and embedded environments. VxWorks is more expensive than open-source alternatives such as Linux variants (many variants of Linux are free, while VxWorks licenses cost on the order of $\$ 10,000$ USD [21]), but VxWorks also has more comprehensive support from the manufacturer. In our system design, VxWorks performs additional processing after the transpond detection and 
decoding provided by the custom hardware. Figure 2.16 shows how VxWorks fits into the overall system block diagram to perform additional data processing.

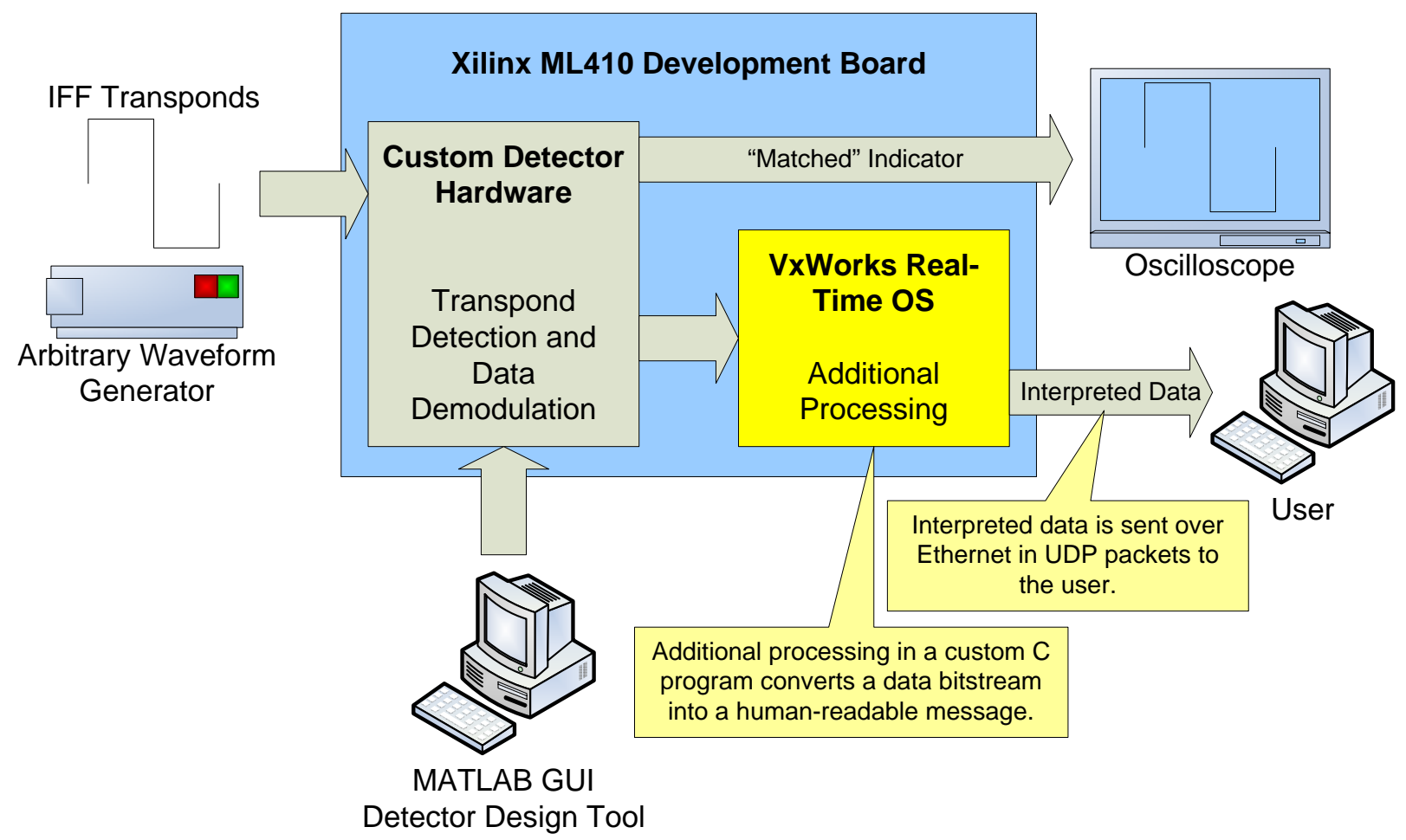

Figure 2.16: The VxWorks Real-Time Operating System Performs Additional Data Processing The VxWorks environment runs on the PowerPC processor, part of the FX60 chip on the Xilinx ML410 development board. Raw data extracted from a transpond message by the detector hardware is converted via a lookup table into human-readable messages and sent to the user over Ethernet.

VxWorks is a UNIX-like environment that allows the user to execute $\mathrm{C}$ code directly from the shell without pre-compilation. A screenshot of the VxWorks terminal is shown in Figure 2.17. We chose to use a program running in VxWorks to interpret, format, and send the extracted IFF data to the user over Ethernet and serial. Advantages to working with VxWorks are ViaSat already uses VxWorks in many products and the support provided by WindRiver helps eliminate problems in system 
integration. Since WindRiver already provides support for our chosen hardware platform, the Xilinx ML410 development board, we avoided reinventing the wheel by writing drivers and hardware support that are already available.

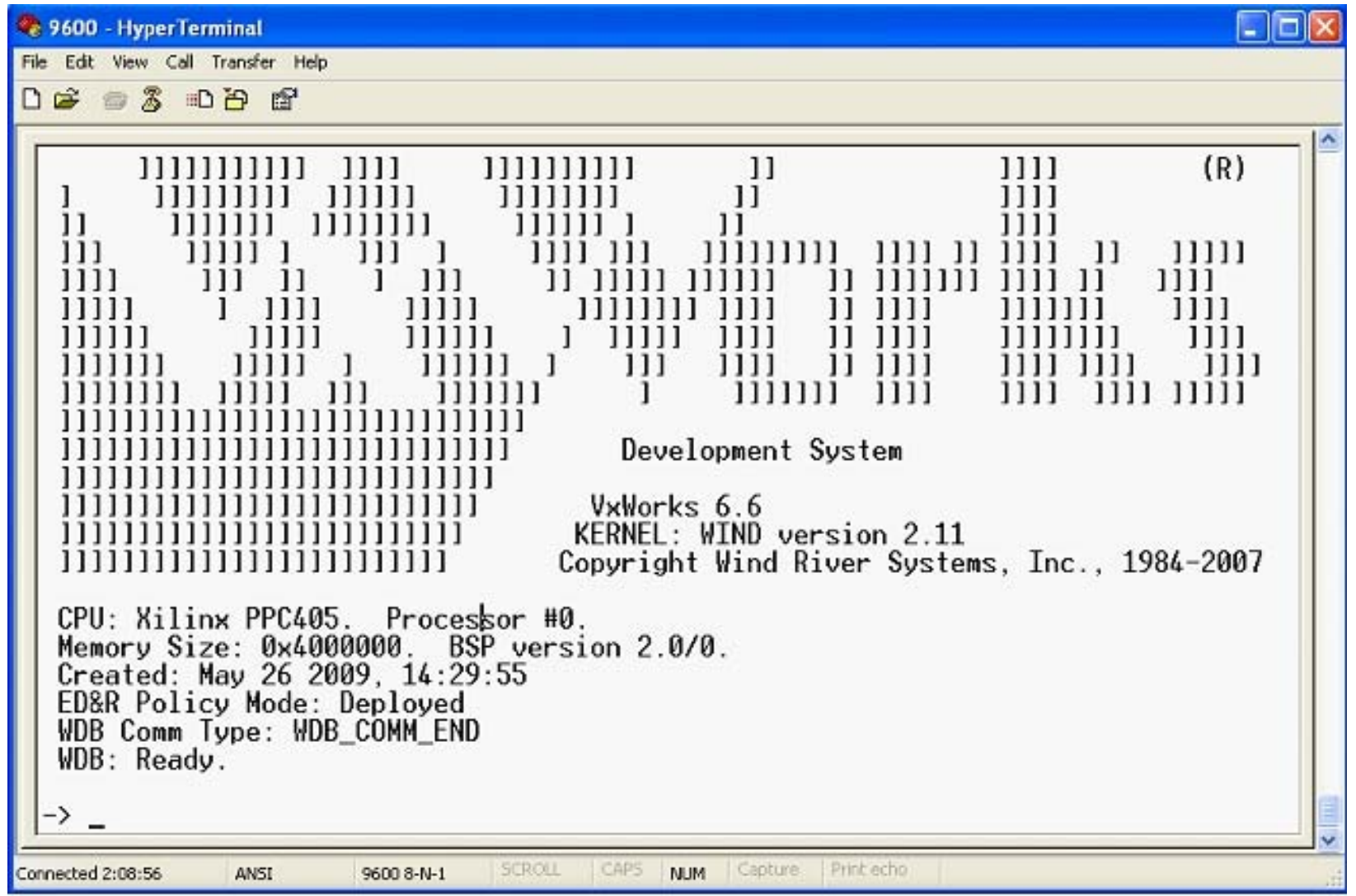

Figure 2.17: The VxWorks Splash Screen Displayed Over the Serial Interface This presence of this splash screen indicates that the boot-up of the VxWorks operating system was successful and the system is up and running. The console in this interface allows the user to directly input commands to the VxWorks system.

VxWorks software development takes place in the WindRiver Workbench software development environment, based on the Eclipse platform. Our program polls the FIFO empty signal, and when the FIFO contains data, a preset amount corresponding to the length of a message is read out. The number of bits to read out 
of the FIFO per message is assumed to be a priori knowledge for this project. This data is then fed into a lookup table to determine what the message actually means, formatted into human-readable ASCII, and sent to the host computer in a UDP packet over Ethernet. The host computer observes these UDP packets with the Wireshark network protocol analyzer program.

The lookup table currently contains only transponds dealing with the altitude of the system under test (SUT). However, the lookup table could easily be extended to cover the entire spectrum of possible Mode S messages defined by the International Civil Aviation Organization (ICAO). Expanding the lookup table was a low priority for this project since our goal was a proof-of-concept rather than a comprehensive solution to detecting and decoding IFF/SSR messages.

One step currently missing from the embedded software is the algorithm necessary to perform the parity check on detected messages. Supporting software in the MATLAB waveform definition GUI would be necessary to define the parity check algorithm that corresponds to different waveforms. The Mode S parity generation algorithm was given to us by ViaSat in C code as an example and could be reversed to perform parity checking. The software should either reject a message as invalid after failing the parity check, or continue sending it to the user as a formatted message if the parity check passes. 


\section{Results}

The detectors designed and implemented by the process described in this project successfully detect and decode the waveforms they are looking for. Conversely, messages that are out of tolerance or incorrectly formatted are ignored by the detector. Pictured in Figure 3.1 is a transpond message in IFF Mark XII Mode S format being successfully detected by the system.

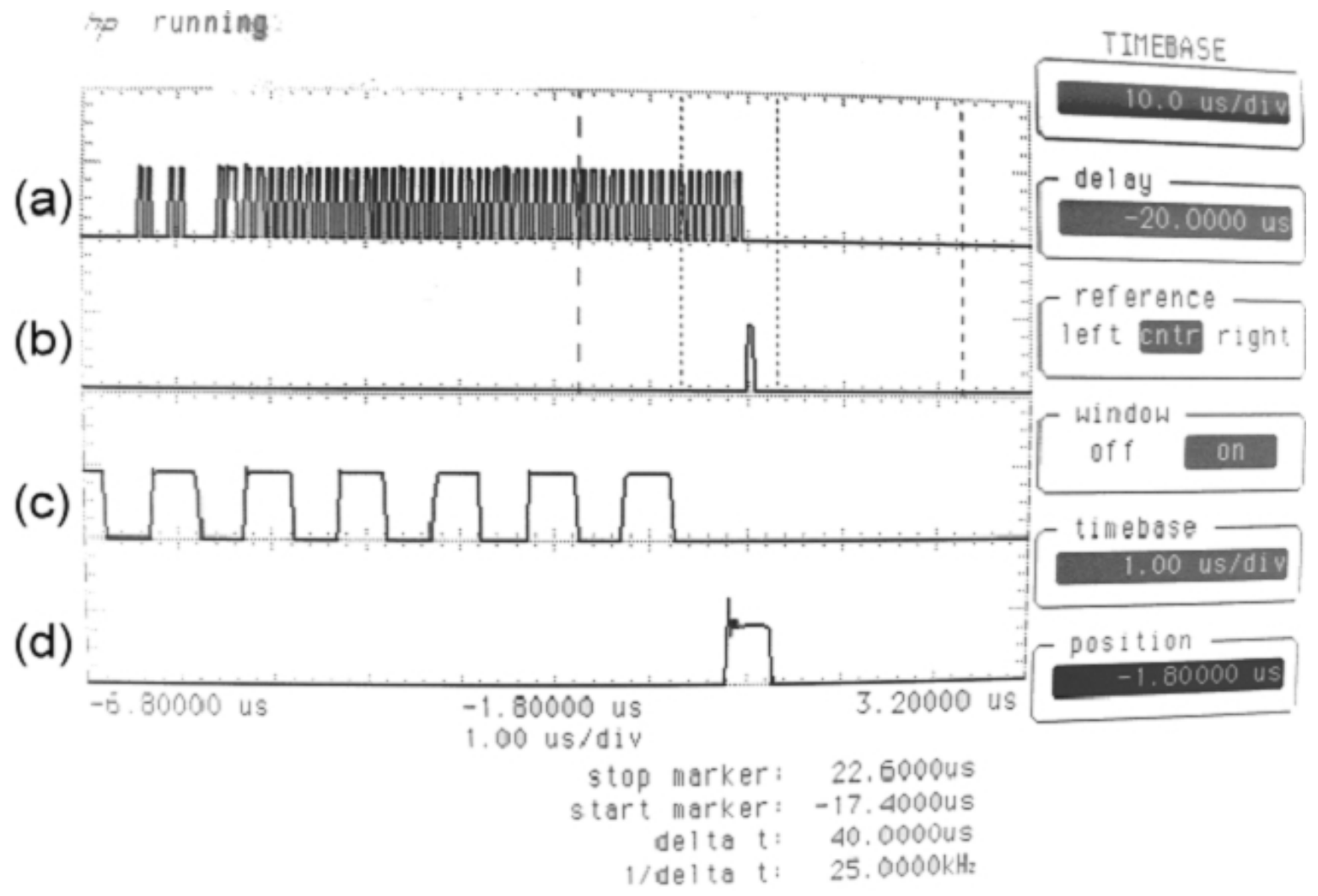

Figure 3.1: Oscilloscope Output Showing IFF Mark XII Mode S Extended Squitter Transpond and "Matched" Flag

(a) IFF Mark XII Mode S Extended Squitter transpond, generated by Agilent 33120A arbitrary waveform generator.

(b) "Matched" flag indicates the message was properly formatted and successfully decoded.

(c) Enlarged view of the last 7 data bits of the message shown in (a).

(d) Enlarged view of the "matched" flag shown in (b). 
There are different ways to move a test input waveform in and out of tolerance. The easiest way is to simply change the operating frequency of the arbitrary waveform generator. However, the current detection algorithm calculates pulse windows based on a timescale relative to the first rising edge of the transpond message. This means that the effects of operating frequency are not constant on all pulses in the test waveform changing the operating frequency of the arbitrary waveform generator moves the last pulses of the waveform more out of tolerance than the first pulses in the waveform. By changing the operating frequency of the arbitrary waveform generator, a detector (specified to a tolerance of $\pm 5 \%$ ) functions within a range of approximately $\pm 1 \%$. Figure 3.2 shows the detector rejecting a transpond waveform that is out of tolerance. 


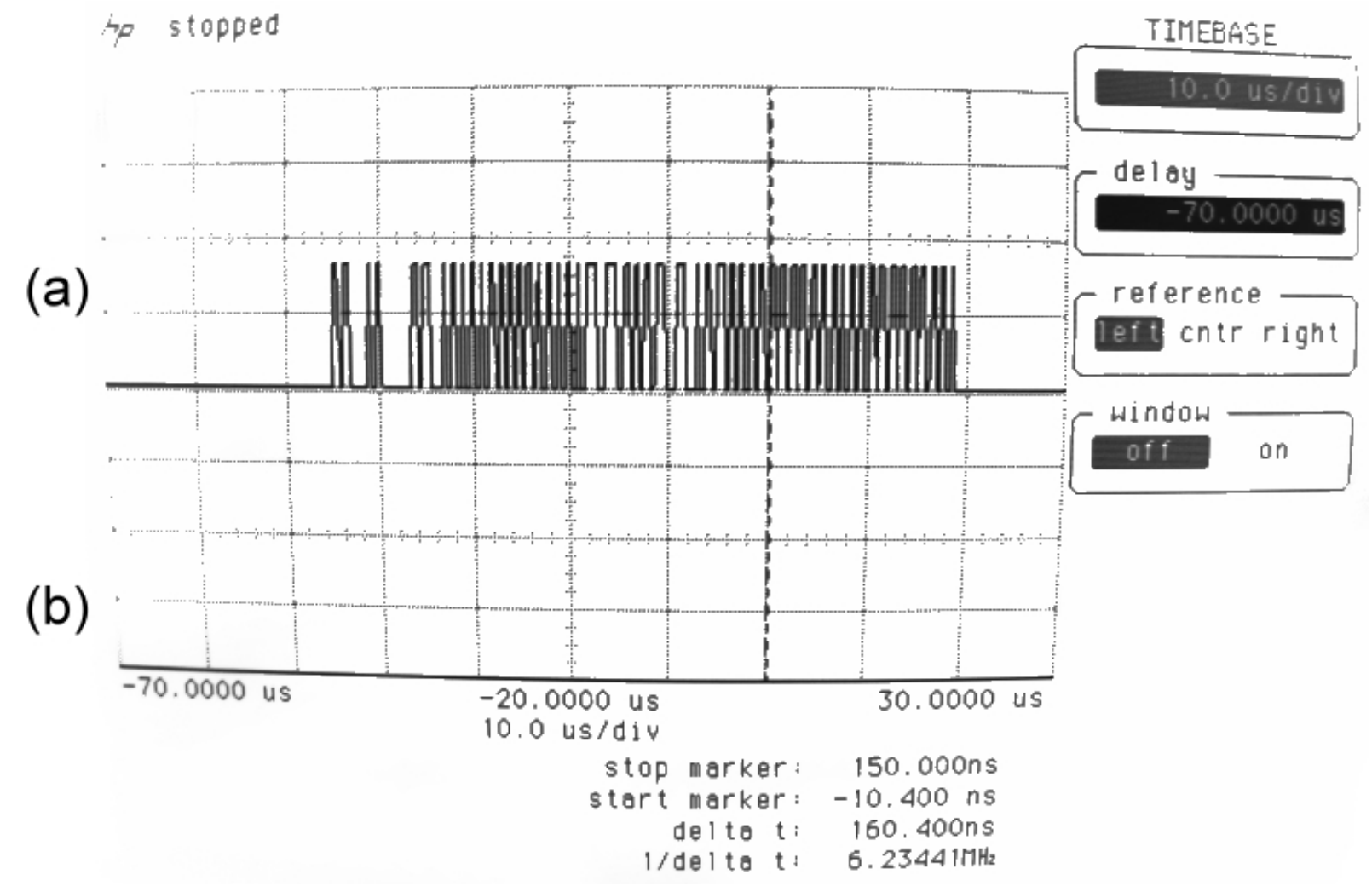

Figure 3.2: Oscilloscope Output Showing IFF Mark XII Mode S Message +7\% Out of Specification and No "Matched" Flag

(a) IFF Mark XII Mode S message +7\% out of specification, generated by Agilent $33120 \mathrm{~A}$ arbitrary waveform generator.

(b) Absence of "matched" flag indicates the message was not properly formatted and/or unsuccessfully decoded.

Another way to change a test waveform in and out of tolerance is to start with a valid waveform, calculate the correct operating frequency and hold this constant, then edit the list of samples contained in the *.csv file to adjust individual pulse widths. This method of adjusting tolerance does not have compounding effects on subsequent pulses in the waveform. A detector (specified to a tolerance of $\pm 5 \%$ ) successfully detects and decodes the data from a message with data bits with pulse widths $\pm 5 \%$ out of specification. An IFF Mark XII Mode S transpond with $+5 \%$ out of specification data pulses is shown being detected in Figure 3.3. 


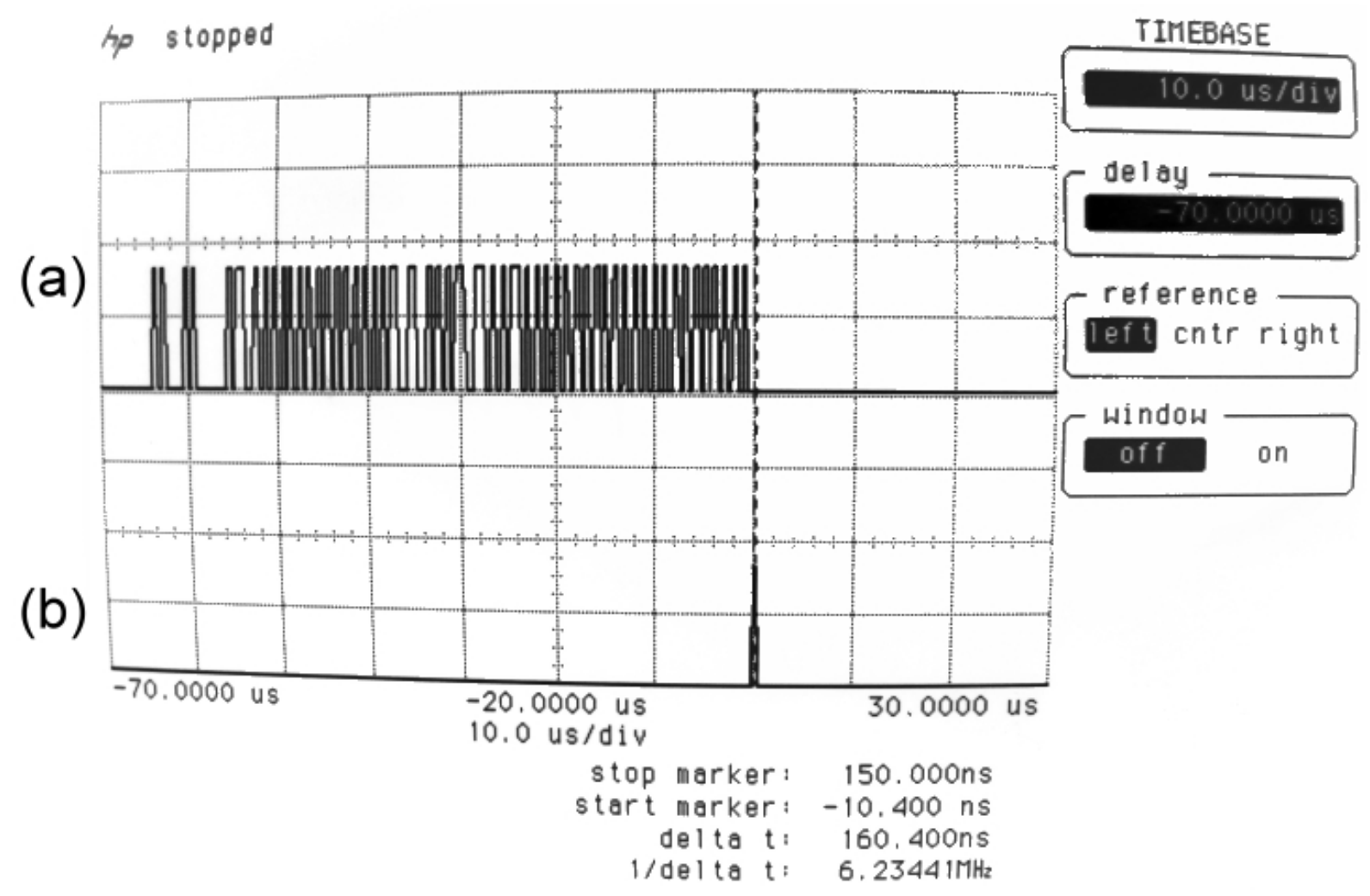

Figure 3.3: Oscilloscope Output Showing IFF Mark XII Mode S Message + 5\% Out of Specification and "Matched" Flag

(a) IFF Mark XII Mode S message $+5 \%$ out of specification, generated by Agilent $33120 \mathrm{~A}$ arbitrary waveform generator.

(b) "Matched" flag indicates the message was within tolerance and data successfully decoded.

Figure 3.4 shows the VxWorks terminal output generated by successfully detected Mode $\mathrm{S}$ transponds. The data shown in the console output is also sent over Ethernet to the host PC. The UDP packets send by the software running in VxWorks were successfully observed in the Wireshark network analysis program. One current issue with the software is that the data length of messages seen by the detector is input as a priori knowledge to the software. Consequently, if the data in the FIFO falls out of sync with the blocs of data the software is reading out, the data interpreted by the software 
will be garbage. Fixing this condition and other improvements to the detector system are described in Section 5, Future Work.

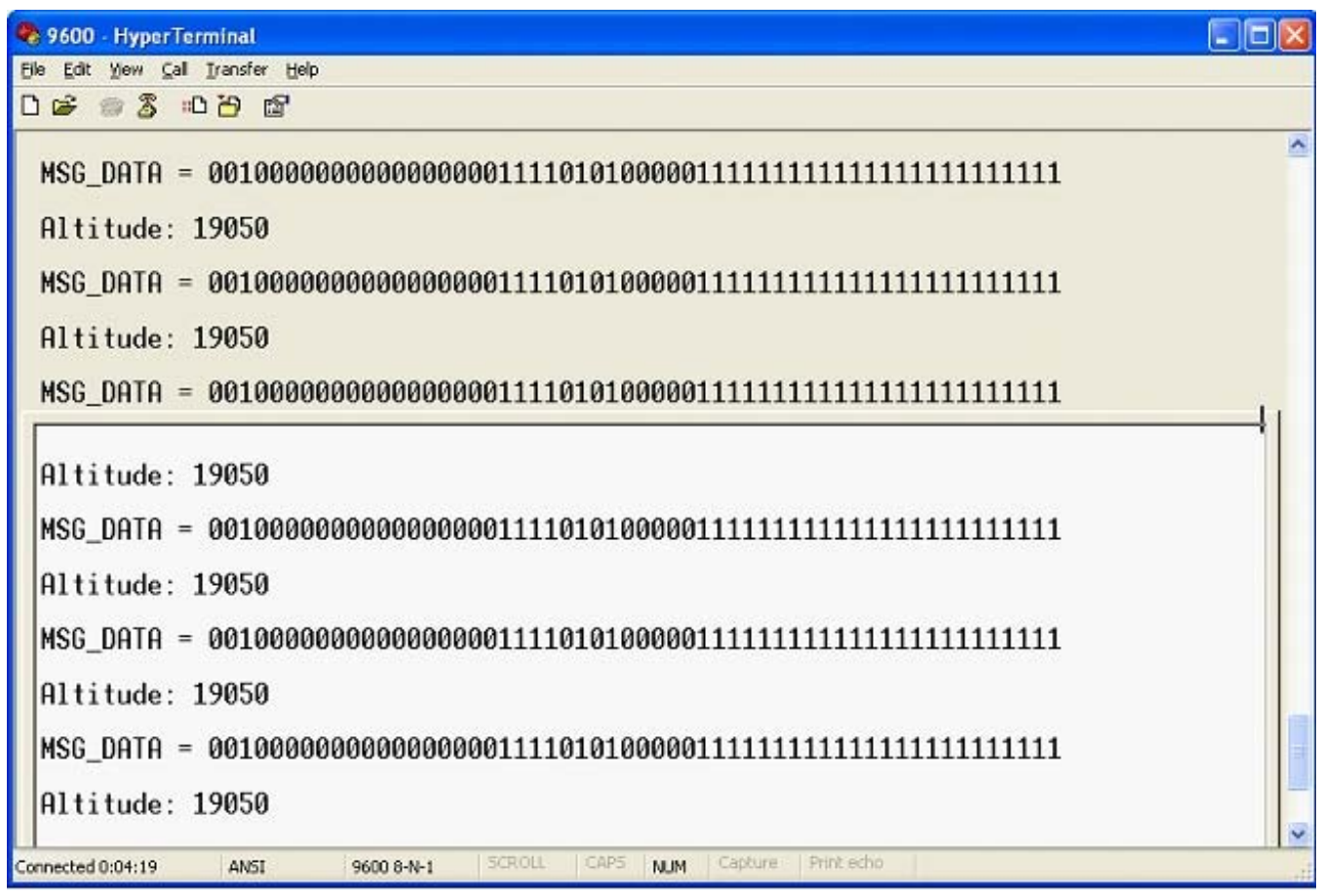

Figure 3.4: VxWorks Terminal Output Showing Detected IFF Mark XII Mode S Messages The "MSG_DATA" field displays the data bit sequence of the detected message. The "Altitude" field displays the interpreted altitude value that the data bit sequence represents. Each time an altitude value is displayed, UDP packets containing both the "MSG_DATA" and altitude values are sent over Ethernet to a host computer.

The results from testing the individual detectors generated by the design process described in this project demonstrate successful operation of the intended designs: properly formatted waveforms are detected while improperly formatted waveforms are rejected. Once a transpond is detected, the system achieved the goal of processing and sending the data to a user at a separate terminal. These successes in the design process and integration techniques are a proof-of-concept demonstration for both our sponsor, ViaSat, as well as the general development community. 


\section{Conclusions}

Despite some shortcomings of the System Generator software as a framework described at the end of Section 2.2, abstracting the design process to a graphical level has enormous potential benefits for developers. A simplified design process leads to reduced costs and development time for prototypes and an overall faster turnaround time for products. Designers should consider at least some level of simplifying the design process: weighing the potential gains against the time investment in creating the abstraction layer. Following the design process described in this document to its end yields a hardware module in the form of a "black box" peripheral that can be attached to a multitude of designs. Using a similar process to generate other custom peripherals can be used in a wide variety of engineering applications including digital signal processing systems and communications.

The IFF detector implementation proved to be a success: properly formatted messages were correctly demodulated, incorrectly formatted messages were rejected by the detector, and the system design flow was proved at a proof-of-concept level. Initial testing of the design was very quick in Simulink and Xilinx System Generator, rather than having to go through the entire HDL synthesis process each time an algorithm changed. After the initial time investment of writing the GUI and export functions, the design and implementation process for any individual detector hardware is reduced from a matter of hours to a matter of minutes. Of course, testing and verification of the design in hardware is still required. 
At the conclusion of the project, we leave ViaSat and any potential future Cal Poly project team members with the source code for the waveform definition MATLAB GUI and export function, several saved detector waveforms, the Simulink model for use with Xilinx System Generator, the Xilinx Platform Studio project files for the system, and the VxWorks software build including our custom processing program. Also included in the project files is a test waveform generator program that can be used to set up the arbitrary waveform generator. Included in Appendix D: User's Guide is a comprehensive walkthrough for designing and implementing a detector in hardware. 


\section{FUTURE WORK}

Future work on this project might focus on generalizing the abstracted design process to a wider array of applications. Companies like Xilinx that already manufacture products similar to System Generator may continue to add abstraction layers within their existing software, while end-users might continue to build abstraction layers on top of the provided frameworks. Abstracted development environments have already proven valuable in reducing development time, costs, and training - therefore, a study of the value provided by additional abstraction layers on top of products like Xilinx System Generator would give insight into any possible diminishing or exponential returns.

Future work specific to the SSR transpond detector could include reinstating the temporary data register, implementing an interrupt-driven software interface, adding the parity check algorithm to the embedded software, and eliminating the need for a priori knowledge of the length of a message by the software. Note that such future improvements could be made either in the development environment or on the custom ViaSat hardware in a more polished final product.

Reinstatement of the temporary data register has the advantage of eliminating the possibility of garbage data being written to the FIFO in the event of a message falling out of tolerance during the middle of a packet. This fix would require either bug fixes from Xilinx to the System Generator or Platform Studio software packages or a clever workaround to the shortcomings of indexing memory arrays in M-code interpreted by 
System Generator. The detector export source code includes both the workaround and commented out code supporting the temporary data register and writing data to the FIFO in the output stage of the FSM.

An interrupt-driven software interface would, rather than polling the empty flag of the FIFO, receive an interrupt from the FSM after a correctly-formatted transpond is detected. Moving to an interrupt-driven software scheme eliminates the need for polling and reduces CPU usage during idle times and freeing the VxWorks operating system to work on other processes.

The parity check algorithm could be implemented in either hardware within the detector FSM or in the embedded software. This project hoped to implement parity checking in software based on ease of implementation and availability of example C code from ViaSat that performed the parity generation. Another facet of adding the parity check would be the need to define how parity is computed in the waveform definition GUI, and then this would be converted to either HDL or C to actually perform the algorithm. The parity check is a required step in the validation of a SSR transpond and should be added in a way that future developers feel is most appropriate.

Currently, the embedded software relies on a priori knowledge of the length of detected messages to read out the correct number of bits from the FIFO. This requirement should be eliminated in order for the software to be compatible with detector hardware looking for messages of varying lengths. Possibilities for this improvement 
include automatically generating software that goes with the desired waveforms at the same time as the HDL is generated, or perhaps changing the FIFO from one-bit wide to a width corresponding to an entire message word length. The obvious disadvantages to widening the FIFO are that the GPIO IP-core can only handle a maximum width of 32 bits, and adjustments in EDK to the GPIO to custom detector interface would be necessary for each new detector. 


\section{BIBLIOGRAPHY}

[1] “Xilinx System Generator v2.1 for Simulink Reference Guide,” Xilinx, Inc., Available: https://safe.nrao.edu/wiki/pub/CICADA/WebHome/xilinx_ref_guide.pdf [Accessed: 9 June 2009].

[2] "Identification, Friend or Foe (IFF)," in Federal Standard 1037C: Glossary of Telecommunications Terms, Boulder, CO: National Telecommunications and Information Administration, 7 August 1996, Available: http://www.its.bldrdoc.gov/fs-1037/dir-018/_2649.htm [Accessed: 12 July 2009].

[3] Vabre, Phil, "Air Traffic Services Surveillance Systems, Including an Explanation of Primary and Secondary Radar," Victoria, Australia: The Airways Museum \& Civil Aviation Historical Society, Available: http://www.airwaysmuseum.com/Surveillance.htm [Accessed: 12 July 2009].

[4] Chang, Emily, and Hu, Roger, et al, "The Story of Mode S: An Air Traffic Control Data-Link Technology," Cambridge, MA: Massachusetts Institute of Technology, 15 December 2000, Available: http://mit.edu/6.933/www/Fall2000/mode-s/index.html [Accessed: 11 March 2009].

[5] Stamper, Wes, "Understanding Mode S Technology," RF Design, 1 December 2005, Available: http://rfdesign.com/military_defense_electronics/radio_understanding_mode_technology/ [Accessed: 12 March 2009].

[6] "Mode S: ICAO 24 Bit Aircraft Addresses," London, England: Civil Aviation Authority, Available: http://www.caa.co.uk/default.aspx?catid=810\&pagetype=90\&pageid=6907 [Accessed: 12 July 2009].

[7] Rowley, Jeff, "California Polytechnic State University, San Luis Obispo, Project Based Learning Institute: Transponder Processor," ViaSat, Inc., 16 November 2007.

[8] Kraus, Theresa L., The Federal Aviation Administration: A Historical Perspective, 1903-2008, Washington, D.C.: U.S. Department of Transportation, 2008, Available: http://www.faa.gov/about/history/historical_perspective/ [Accessed: 10 March 2009].

[9] "Death in the Air," Time, 26 December 1960, Available: http://www.time.com/time/magazine/article/0,9171,895134,00.html [Accessed: 11 July 2009].

[10] Wolff, Christian, "Radar Basics - Secondary Radar - FRUIT," Radar Basics, Available: http://www.radartutorial.eu/13.ssr/sr14.en.html [Accessed: 12 July 2009].

[11] Annex 10 to the Convention on International Civil Aviation - Aeronautical Telecommunications Systems - Volume IV: Surveillance Radar and Collision Avoidance Systems, $3^{\text {rd }}$ Edition, International Civil Aviation Organization, July 2002.

[12] Wolff, Christian, "Radar Basics - SSR Mode S - Why Mode S?," Radar Basics, Available: http://www.radartutorial.eu/13.ssr/sr20.en.html [Accessed: 12 July 2009]. 
[13] “Mode S Technical Overview," Eurocontrol, 11 July 2008, Available: http://www.eurocontrol.int/msa/public/standard_page/modes_tech_overview.html [Accessed: 12 July 2009].

[14] “About ViaSat," ViaSat, Inc., 2009, Available: http://www.viasat.com/ [Accessed: 10 June 2009].

[15] "RF Signal Stimulation: Assisting with the Testing of Sophisticated Communications Systems," ViaSat, Inc., 2007, Available:

http://www.viasat.com/files/assets/web/datasheets/rf_signal_stimulation_datasheet_003.pdf [Accessed: 5 July 2009].

[16] "Edwards Air Force Base, Benefield Anechoic Facility," AECOM, Available: http://www.dmjmhn.aecom.com/MarketsAndServices/39/54/index.jsp [Accessed: 10 June 2009].

[17] “Testing Studies Global Hawk's Electromagnetic Profile," The North Spin, 14 July 2008, Available: http://www.thenorthspin.com/news08/071408usaf.html [Accessed 11 June 2009].

[18] Bland, Ivan, and Wu, James, "ViaSat SSR Transponder Decoding," California Polytechnic State University, San Luis Obispo, B.S. Senior Project, 9 June 2008.

[19] “Xilinx ML410 Documentation and Tutorials," Xilinx, Inc., Available: http://www.xilinx.com/products/boards/ml410/index.html [Accessed: 5 July 2009].

[20] "CoreConnect Bus Architecture," International Business Machines Corp., Available: http://www03.ibm.com/technology/power/licensing/coreconnect/index.html [Accessed: 11 June 2009].

[21] Rowley, Jeff, “RE: VxWorks Question,” Personal E-mail (16 July 2009). 
AOPA - Aircraft Owners and Pilots Association

ATCRBS - Air Traffic Control Radar Beacon System

BSB - Base System Builder

BSP - Board Support Package

CPU - Central Processing Unit

DSP - Digital Signal Processing

EDK - Embedded Development Kit

FAA - Federal Aviation Administration

FIFO - First In, First Out memory

FPGA - Field Programmable Gate Array

FRUIT - False Replies Uncorrelated in Time

FSM - Finite State Machine

GUI - Graphical User Interface

ICAO - International Civil Aviation Organization

JTAG - Joint Test Action Group - An interface used to debug and program digital hardware.

IFF - Identification, Friend, or Foe

Interrogation - The uplink component of an IFF communication, data is requested either in a multicast or addressed message.

ISE - Xilinx Integrated Software Environment

The MathWorks - The company that develops MATLAB and Simulink.

MATLAB - A software environment and programming language for manipulation of matrices and numbers, maintained by The MathWorks.

ML410 - The Xilinx ML410 development board is an FPGA development platform containing an FX60 Virtex-4 FPGA and supporting peripherals. 
Mode A - An identification transmission in IFF/SSR format.

Mode $\mathrm{C}-$ An altitude transmission in IFF/SSR format.

Mode S - Mode Select - A data transmission protocol that extends the capabilities of the IFF/SSR communications system. Mode S messages contain either 56 or 112 data bits. Mode S is backwards compatible with Modes A and C.

Multicast - A message broadcast to all available listeners, rather than to a specific addressee.

PLB - Processor Local Bus

PowerPC - A RISC processor architecture developed by Apple-IBM-Motorola in 1991.

RF - Radio Frequency

RTOS - Real-Time Operating System

SDK - Software Development Kit

SPI - Special Position Identification Pulse

SSR - Secondary Surveillance Radar

SUT - System Under Test

Simulink - A graphical system modeling and simulation tool developed by The MathWorks. Simulink is highly integrated with MATLAB and other components such as Xilinx's System Generator can run within Simulink.

System Generator - A Xilinx product that generates HDL code from a specifically formatted Simulink model.

Transpond - The downlink (reply) component of an IFF communication, data is returned either in a multicast or addressed message.

Transponder - The hardware device as part of an IFF system that sends and receives messages.

Verilog - A hardware description language used to model and implement digital circuits.

VHDL - VHSIC (Very High Speed Integrated Circuits) Hardware Description Language, a hardware description language used to model and implement digital circuits.

VHSIC - Very High Speed Integrated Circuits

ViaSat, Inc. - Sponsor of this project and a provider of digital communications products. 
VxWorks - A real-time operating system developed by WindRiver.

WindRiver - The developer of the VxWorks real-time operating system.

Xilinx - A manufacturer of FPGAs and development software for digital design.

XPS - Xilinx Platform Studio 
APPENDIX B: SOFTWARE LISTING

- MATLAB with Simulink 7.4.0.287 (R2007a)

- WindRiver VxWorks 6.6

- Wireshark 1.0.8

- Xilinx ISE Design Suite 10.1.03

- Xilinx System Generator for Simulink 10.1 
- Agilent 33120A Arbitrary Waveform Generator

- Hewlett Packard HP 54501A Oscilloscope

- $\quad$ PC Running Microsoft Windows XP

- Xilinx ML410 Development Board

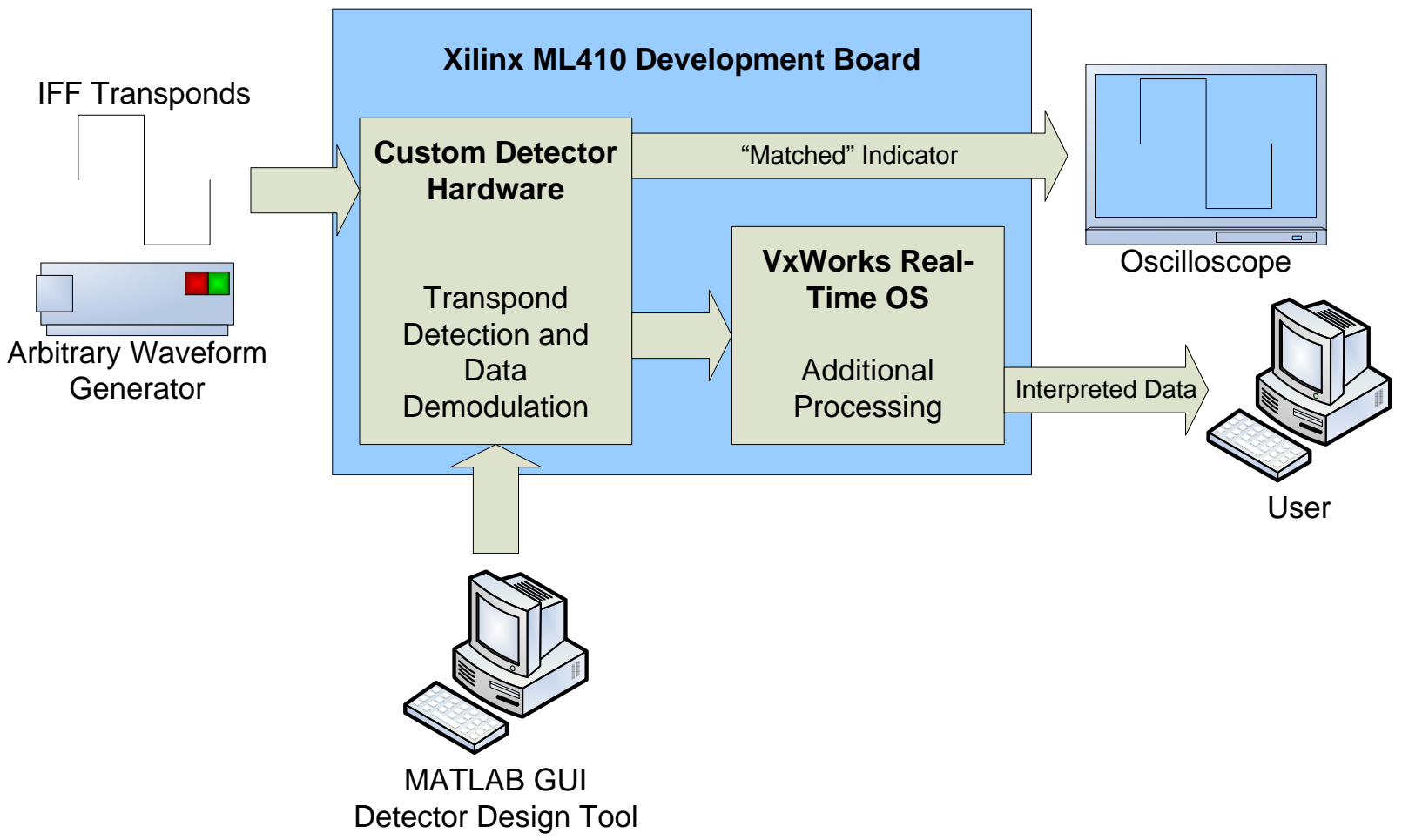

The overall system block diagram shows how the test hardware interacts. The Agilent $33120 \mathrm{~A}$ Arbitrary Waveform Generator produces IFF transponds as test stimuli. The Xilinx ML410 development board houses the detector hardware and VxWorks system. The oscilloscope is used to observe the test inputs and watch for a "matched" indication from the custom hardware. A Windows PC is used in conjunction with MATLAB and Xilinx software to design and implement the detector in hardware, as well as to listen for UDP data packets sent out by software running in the VxWorks environment. 


\section{Overview: Detector Design and Implementation}

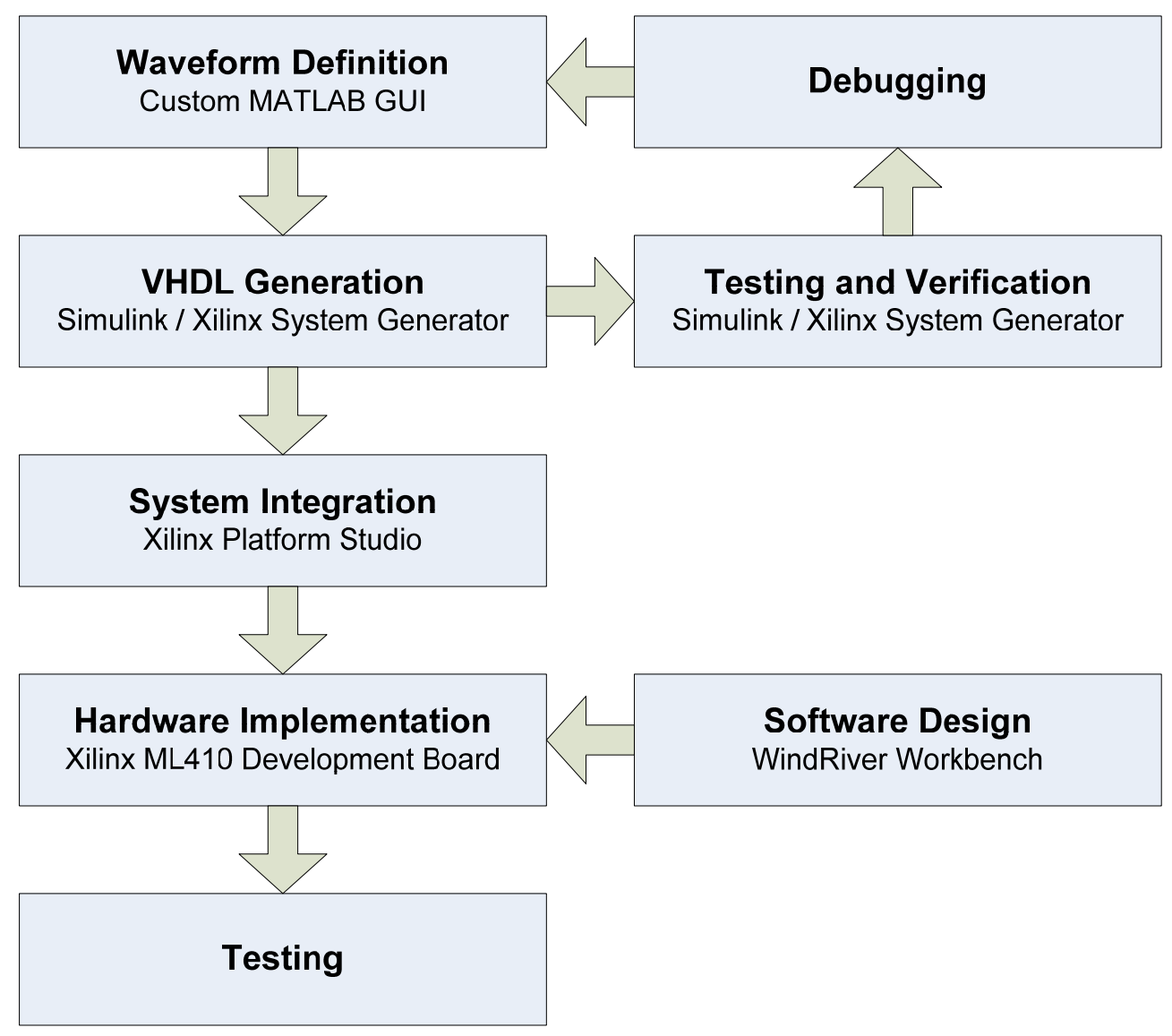

This user's guide is a description of the steps necessary to design an IFF transpond detector and implement the design in the Xilinx Virtex-4 FPGA present on the Xilinx ML410 development board. This detector hardware is implemented in tandem with a VxWorks system running on the FX60 chip's PowerPC processor that performs additional processing. 


\section{Step 1: Define the Waveform in the MATLAB GUI}

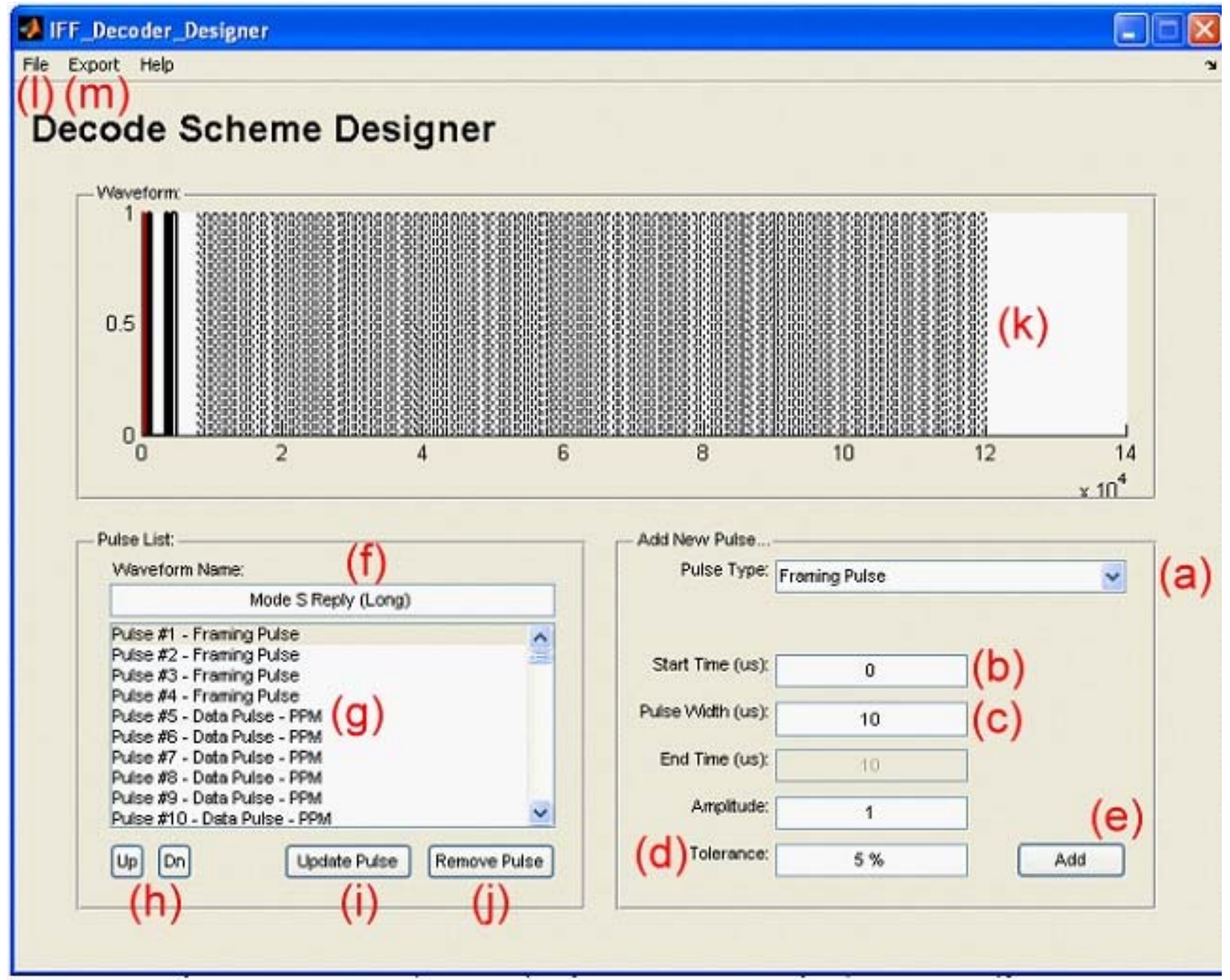

(a) Pulse Type: Pulses can be defined as either a "Framing Pulse" or a "Data Pulse". When "Data Pulse" is selected, a second dialog becomes available to choose the modulation scheme of the data.

(b) Start Time: The start time of the pulse window in microseconds, relative to the first pulse.

(c) Pulse Width: The pulse width of the pulse in microseconds -- or, in the case of pulse position modulated data pulses, the width of the entire window (i.e. twice the actual pulse width). The end time is automatically calculated and displayed based on the Start Time and Pulse Width.

(d) Tolerance: The percentage tolerance the detector should allow. This can be adjusted individually by pulse.

(e) Add: Clicking this button adds a pulse to the waveform with the parameters currently entered in the "Add New Pulse..." frame. 
(f) Waveform Name: When saved, the waveform is identified by this name.

(g) Pulse List: This is a list of the pulses currently in the waveform. Clicking on a pulse in this window highlights it in the waveform display and allows the user to make updates to the selected pulse.

(h) Up and Down: These buttons allow the user to move pulses up and down in the list.

(i) Update Pulse: This button changes the parameters of the currently selected pulse to those entered in the "Add New Pulse..." frame.

(j) Remove Pulse: This button removes the currently selected pulse from the waveform.

(k) Waveform Display: This window shows a graphical representation of the currently loaded waveform. The currently selected pulse is highlighted in red. Framing pulses are indicated by bold lines, while PPM data pulses are indicated by dotted lines.

(1) File Menu: The File Menu contains options for saving and loading waveforms. Waveforms are saved in the *.iff file format, which is really just a different extension over a MATLAB *.mat file.

(m) Export Menu: The Export Menu contains a button to export the current waveform to a detector FSM implemented in MATLAB M-code.

\section{Note:}

Pulses in the pulse list must be ordered chronologically to ensure correct operation of the FSM generated by the export function. 


\section{Step 2: Export the Waveform to M-Code}

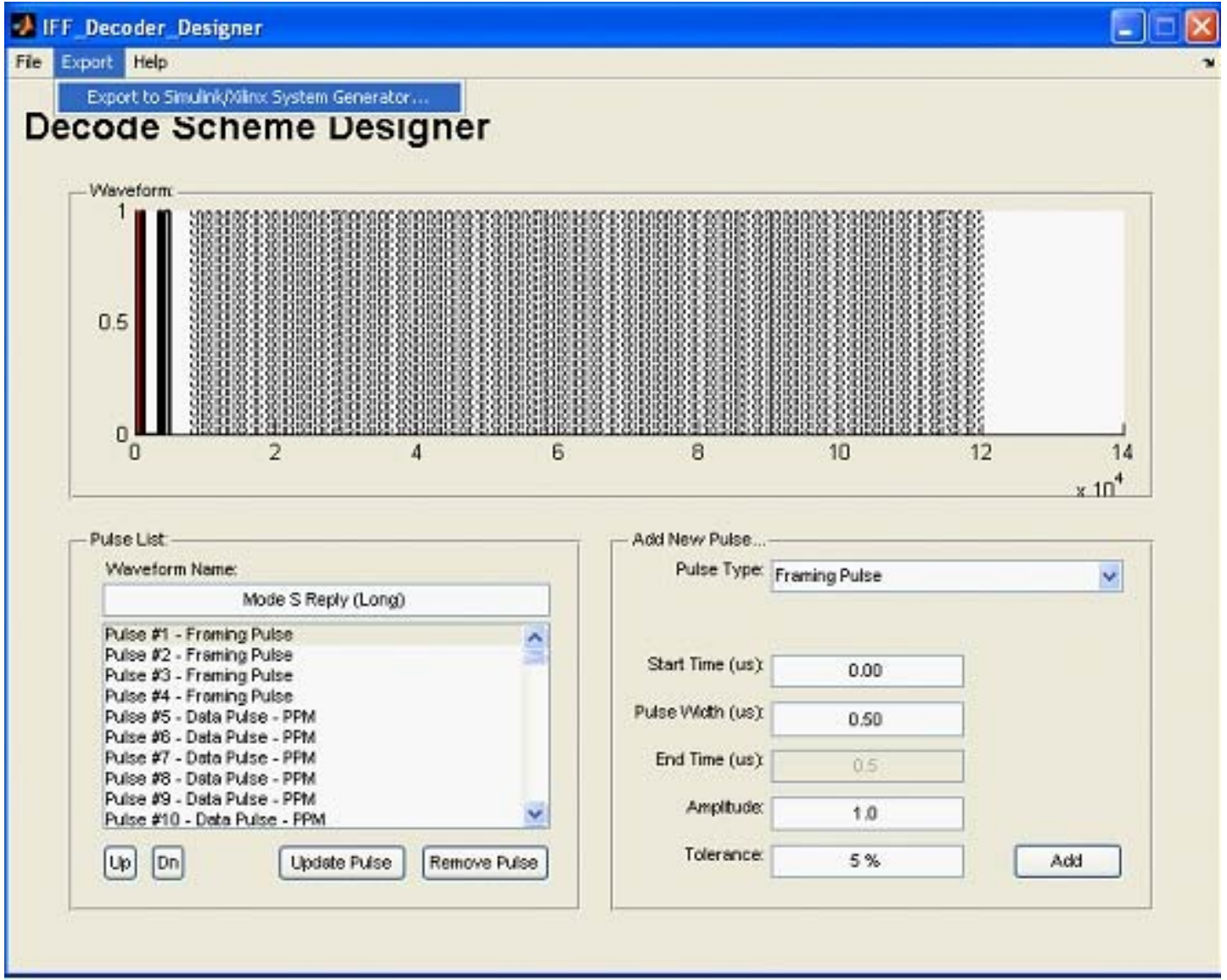

After entering the waveform in the GUI waveform definition tool, export the detector design to M-code by navigating to the "Export" menu and clicking on "Export to Simulink/Xilinx System Generator...". The exported code is saved as "SSR_Reply_DETECTOR.m". 


\section{Step 3: Verify Correct Operation in Simulink / Xilinx System Generator}

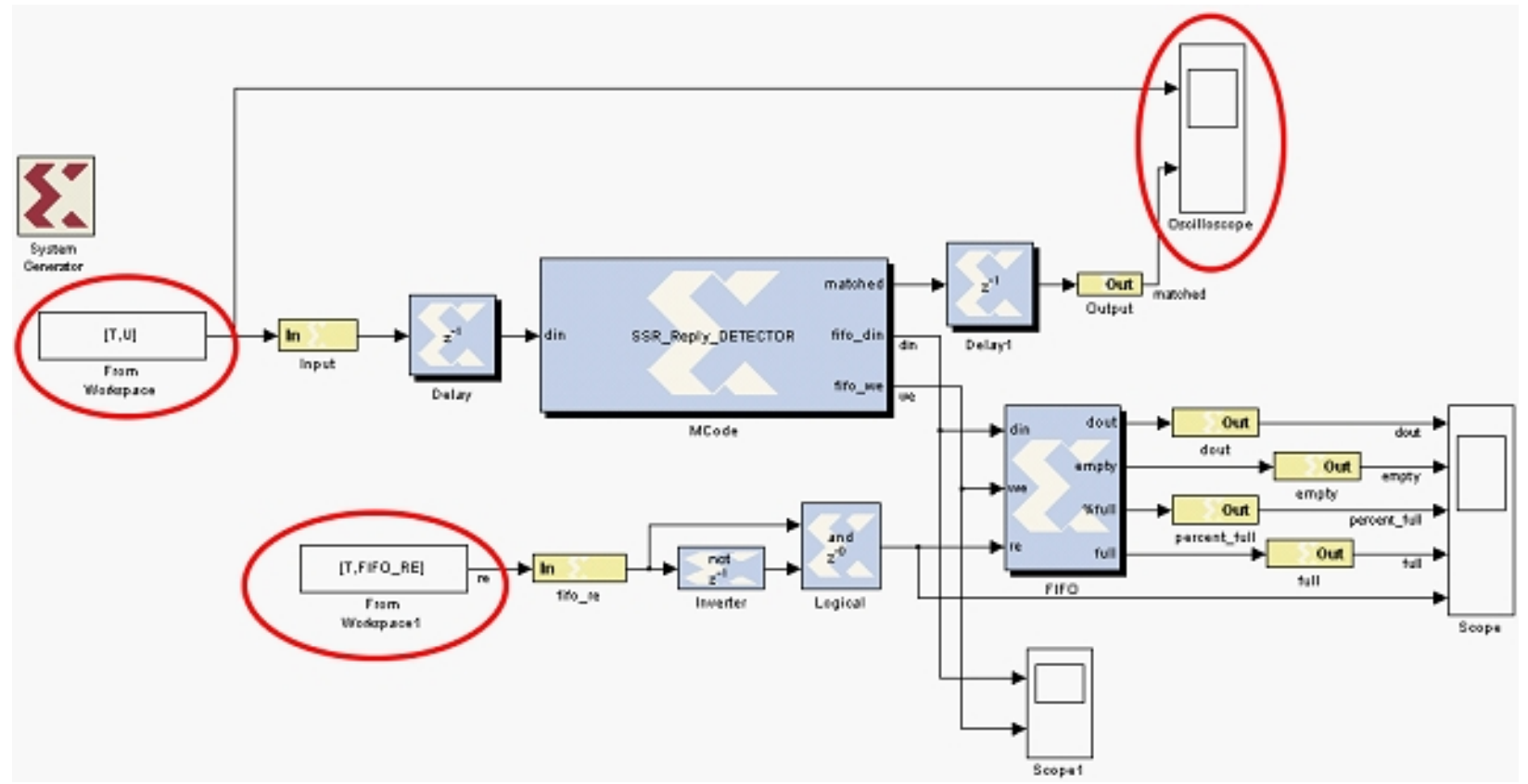

Load test input signals into the MATLAB variables T, U, and FIFO_RE. These signals correspond to a time index, input waveform, and FIFO read-enable signal, respectively. One set of test signals can be loaded by loading "modestestworking-fifo.mat" into the MATLAB workspace.

\section{ly_DETECTOR}

ww Simulation Format Tools Help

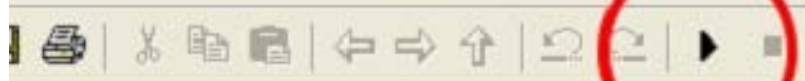

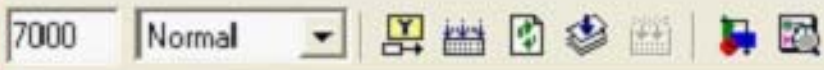

First, make sure that the number of simulation time steps is greater than the length of the test input waveform with room to spare for output states. Then, click on the "Play" button to run the simulation. 


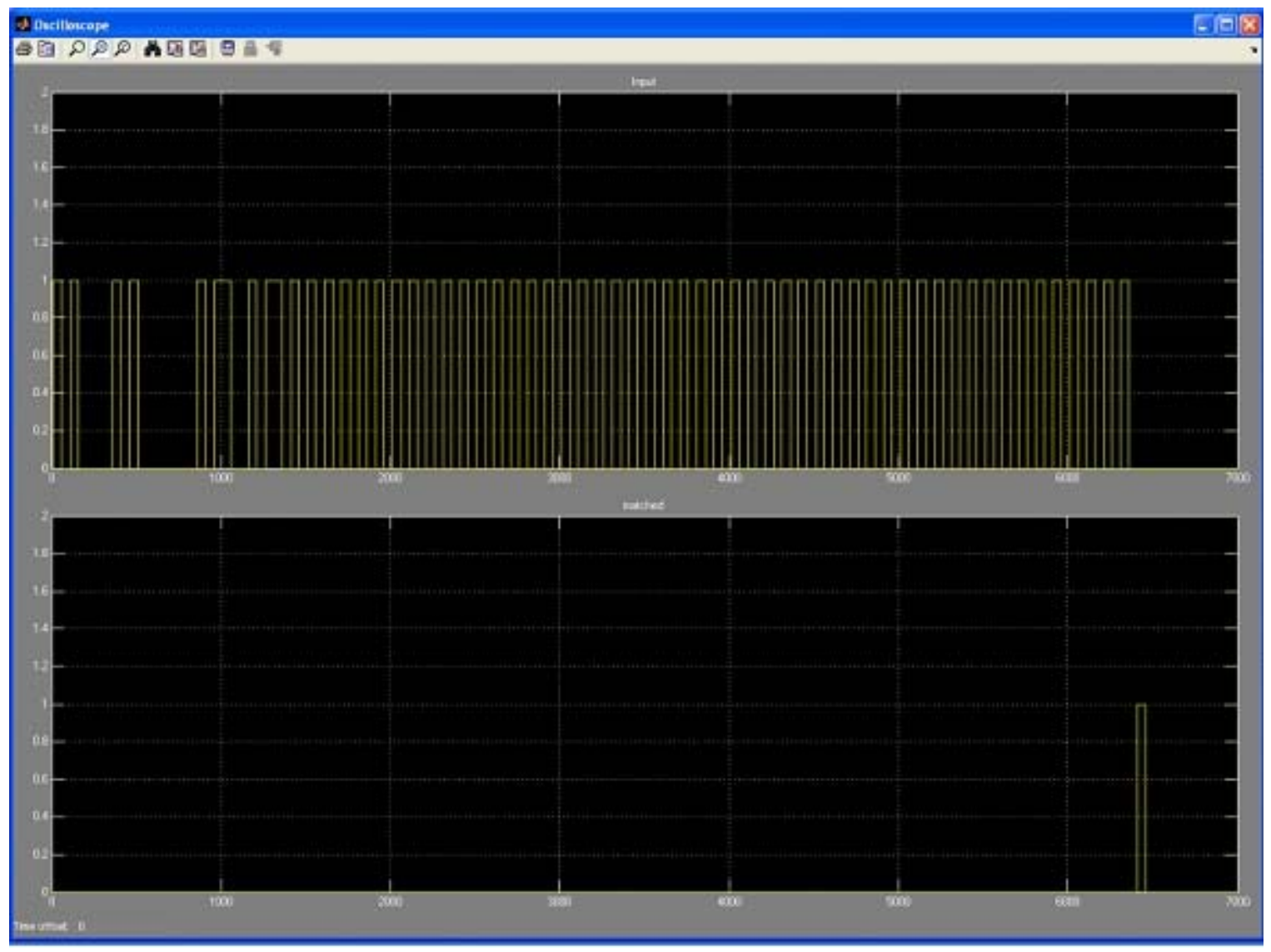

Double-click on the oscilloscope block in the Simulink model to observe the simulated input and "matched" output waveforms. The "matched" signal should go high when a transpond is correctly detected. 


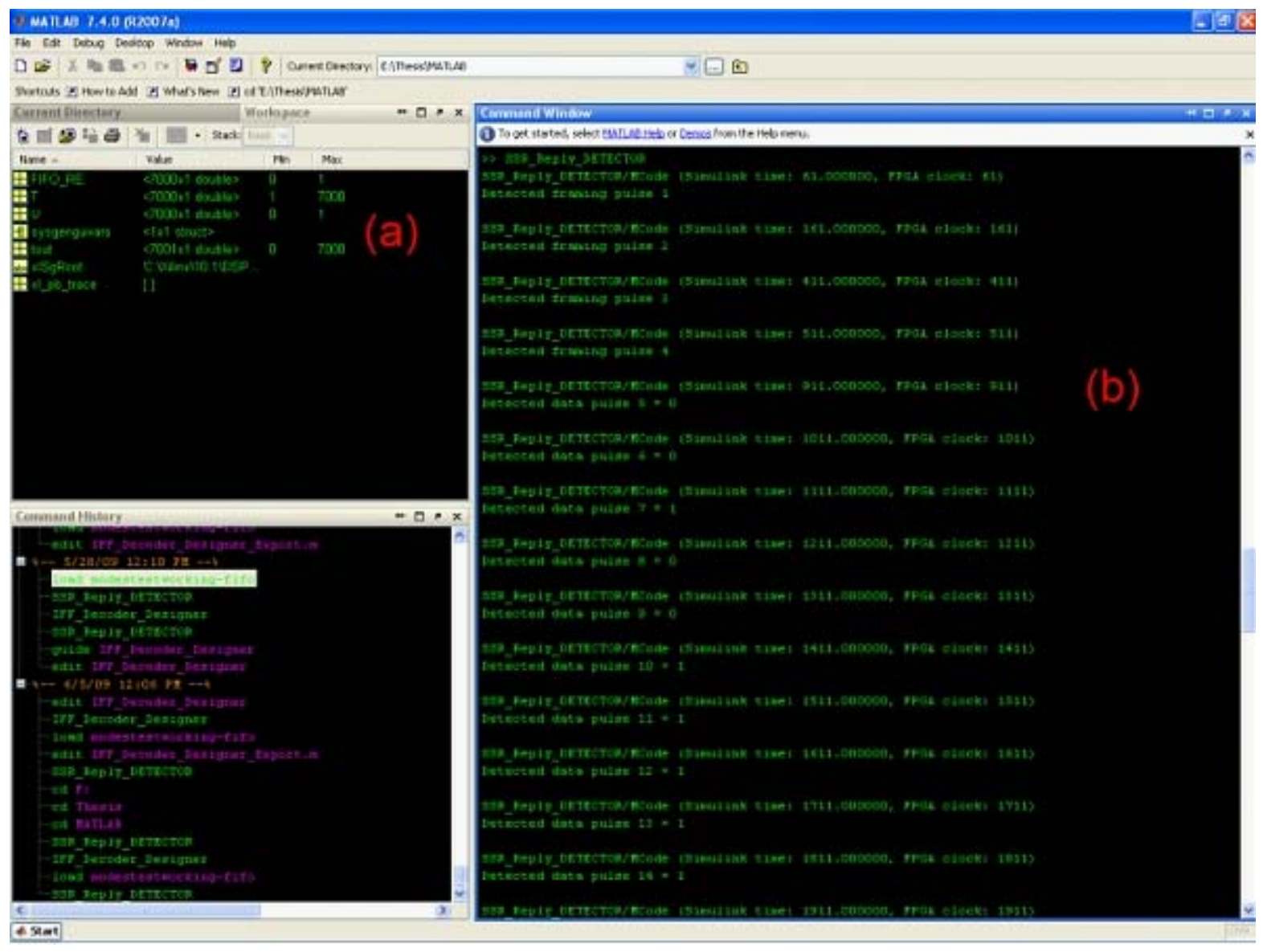

Shown in (a) are the input vectors used by the simulation. After the simulation has completed, detected data bits and other information is displayed on the MATLAB console (b) through use of the "disp()" command. An input flag to the M-code export function controls whether these "disp()" commands are written into the M-code, or not. By default, they are. 


\section{Step 4: Export the Detector Design to HDL}

Double click on the System Generator block in the Simulink Model, "SSR_Reply_DETECTOR.mdl”.

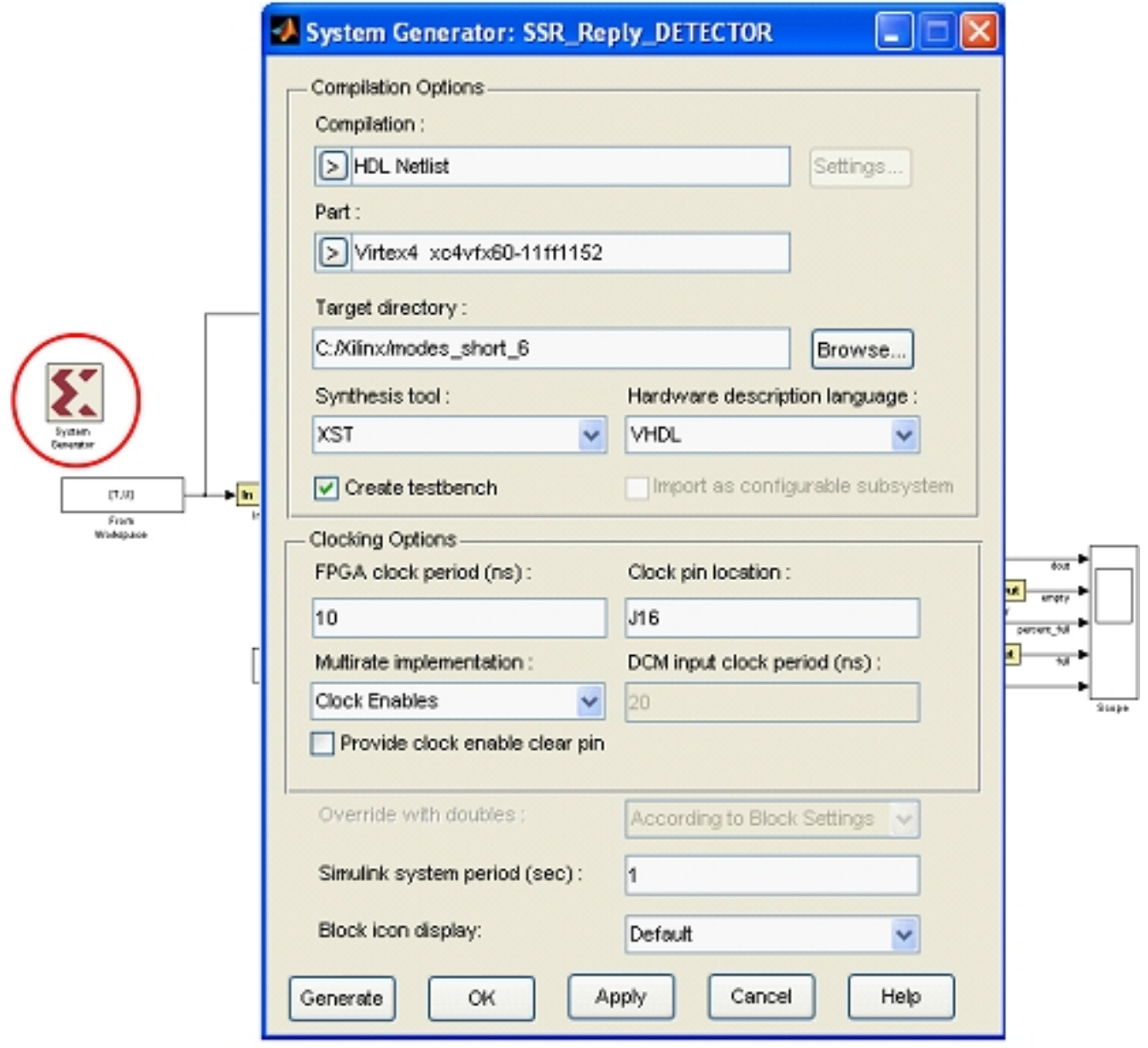

The following options should be set:

Compilation - HDL Netlist

Part - Virtex4 xc4vfx60-11ff1 152

Synthesis tool - XST

Hardware description language - VHDL

FPGA clock period (ns) - 10

Clock pin location - J16

Multirate implementation - Clock Enables

Provide clock enable clear pin - Unchecked

Simulink system period (sec) -1

Block icon display - Default

Change the "Target directory:" to a folder name that you will use to identify this particular detector design. Then, click "Generate". 


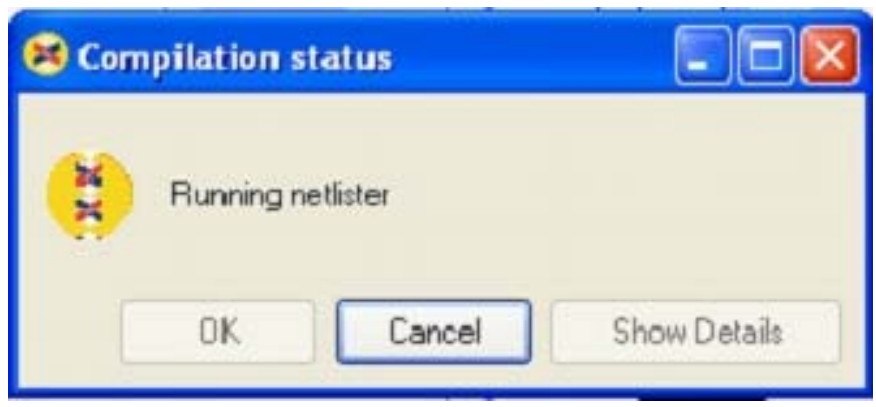

The compilation status dialog will appear and provide an indication when the process of exporting to VHDL is complete.

\section{Step 5: Import the Detector as a Custom Peripheral in Xilinx Platform Studio}

First, open the Xilinx Platform Studio project file. The project file we use is located at "C: $\backslash X i l i n x \backslash$ import_peripheral $\backslash m 1410 \_$ethernet_no_null.xmp".

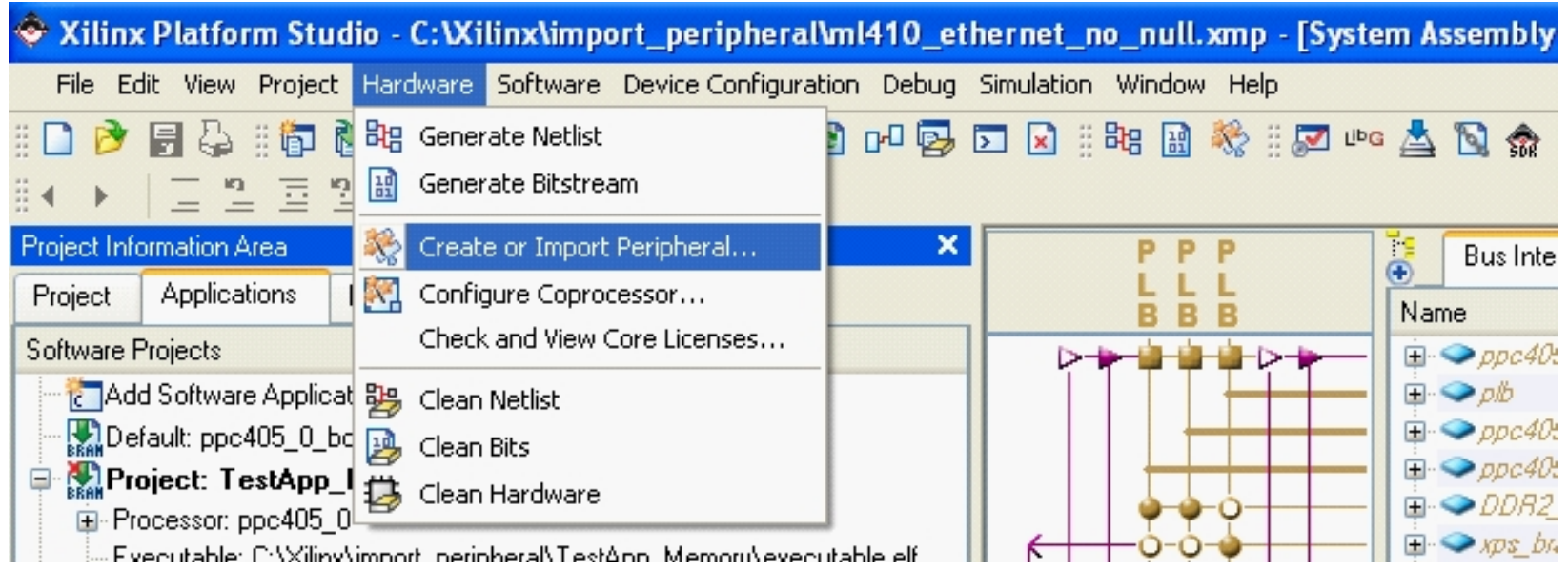

Next, import the detector VHDL module as a custom peripheral by running the "Create or Import Peripheral..." wizard. Click on the "Hardware" menu in Xilinx Platform Studio and select "Create or Import Peripheral..." 


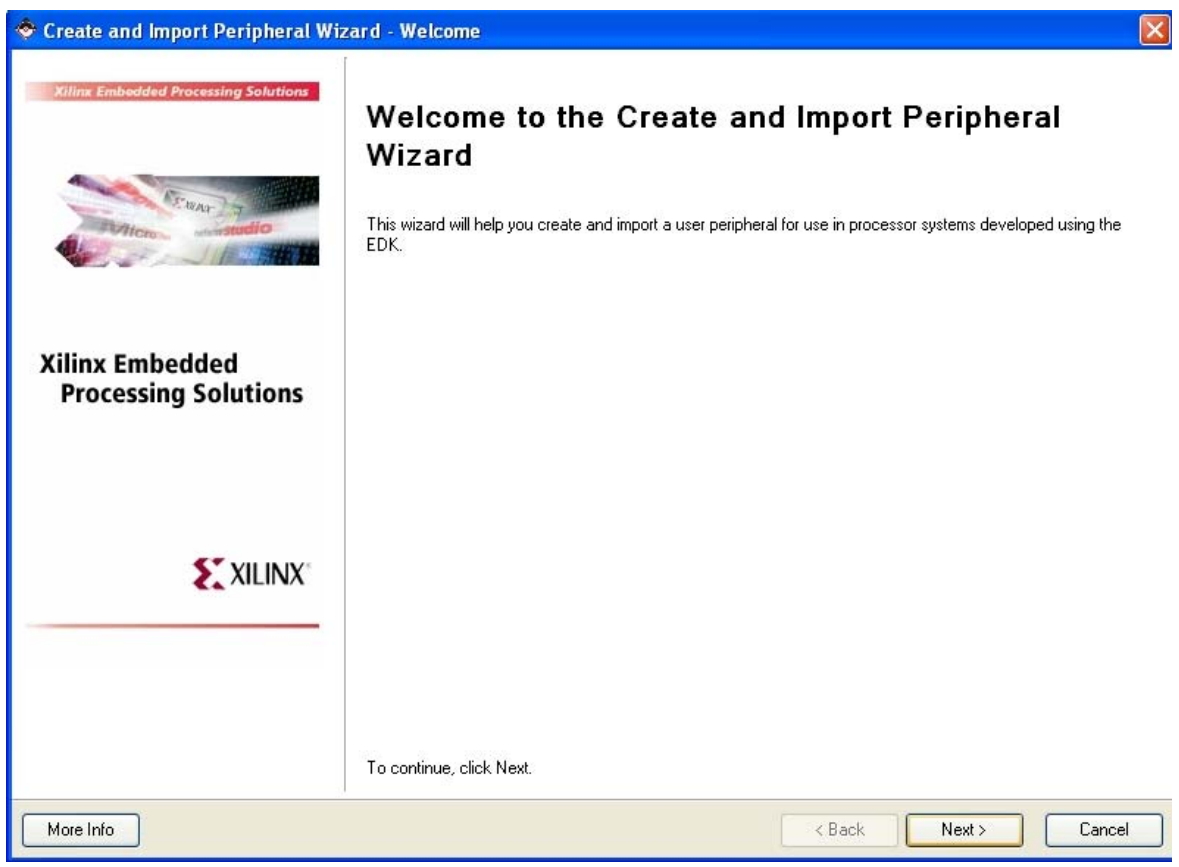

Click "Next" on the first screen of the Create and Import Peripheral Wizard.

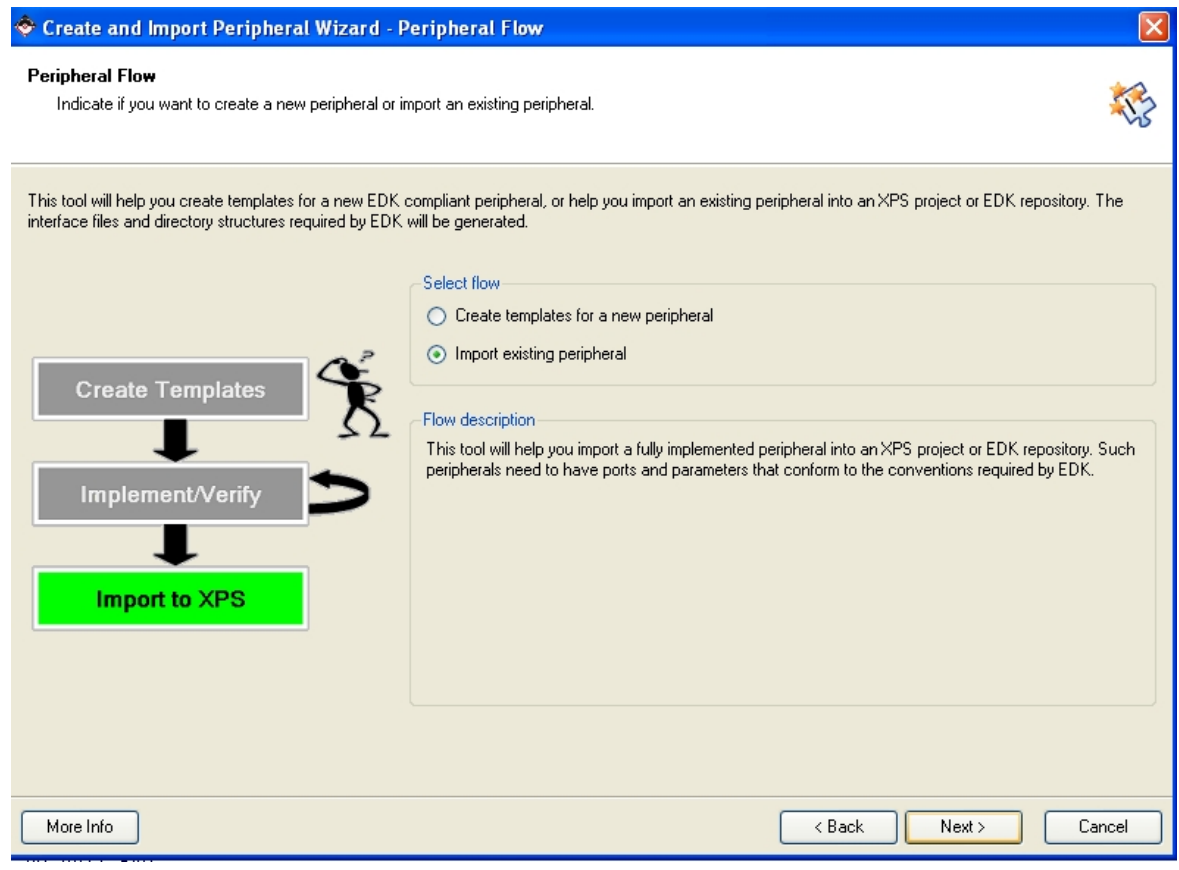

Select "Import existing peripheral" and click "Next". 


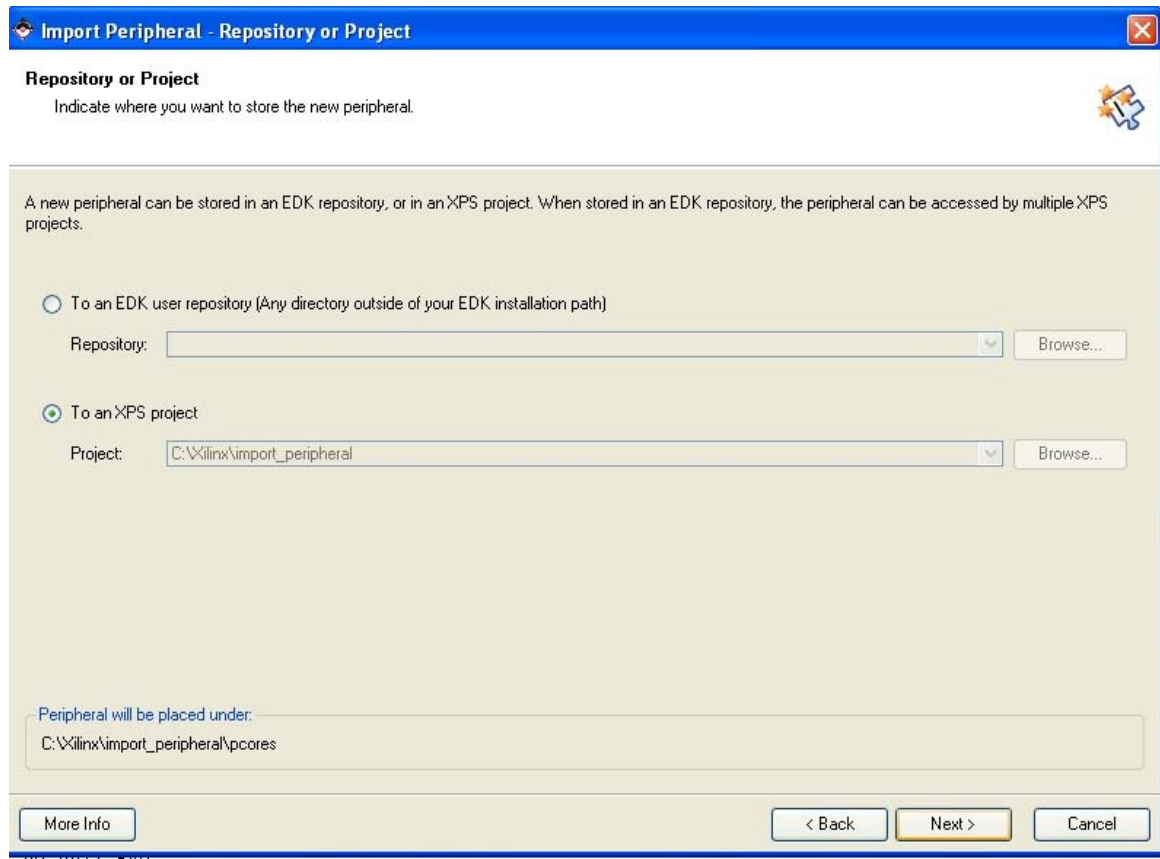

Make sure "To an XPS project" is selected. The grayed out project path should be the current project. Click "Next".

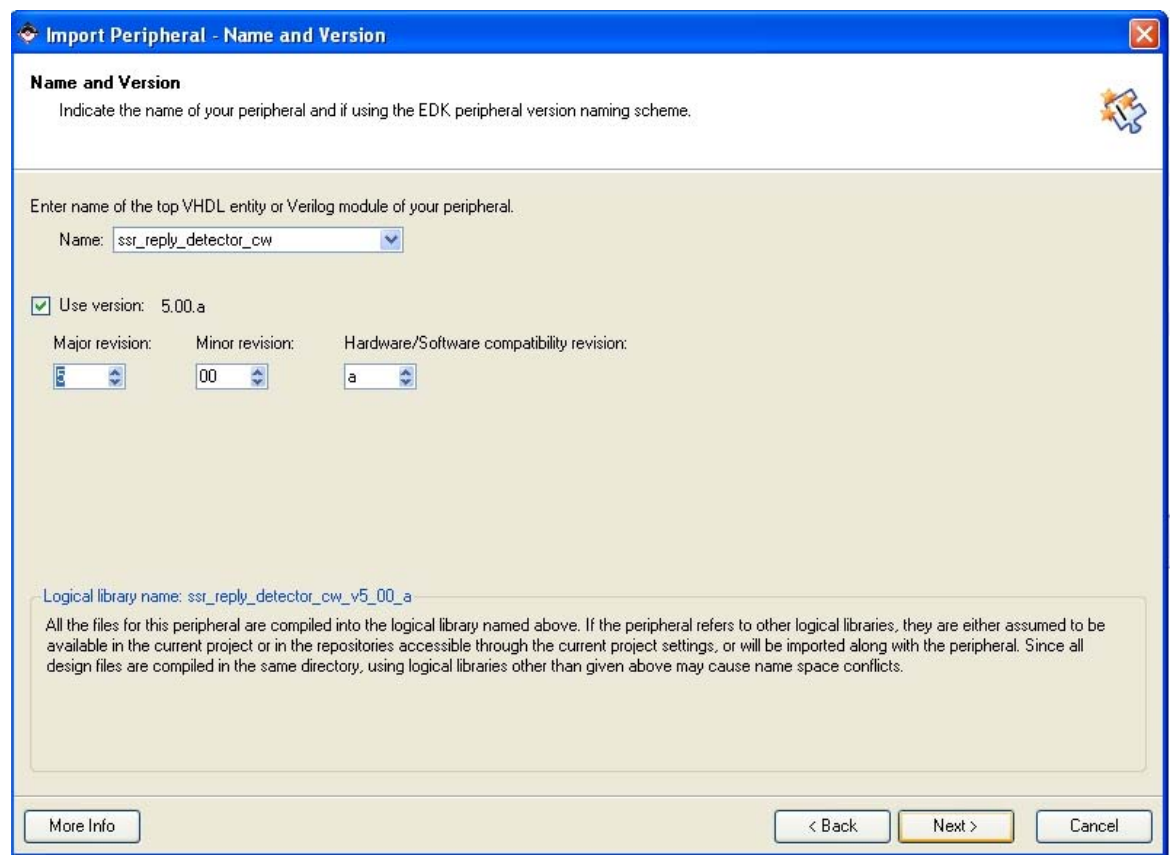

Change the VHDL entity name to "ssr_reply_detector_cw". Select an appropriate version number for the custom peripheral, if desired. 


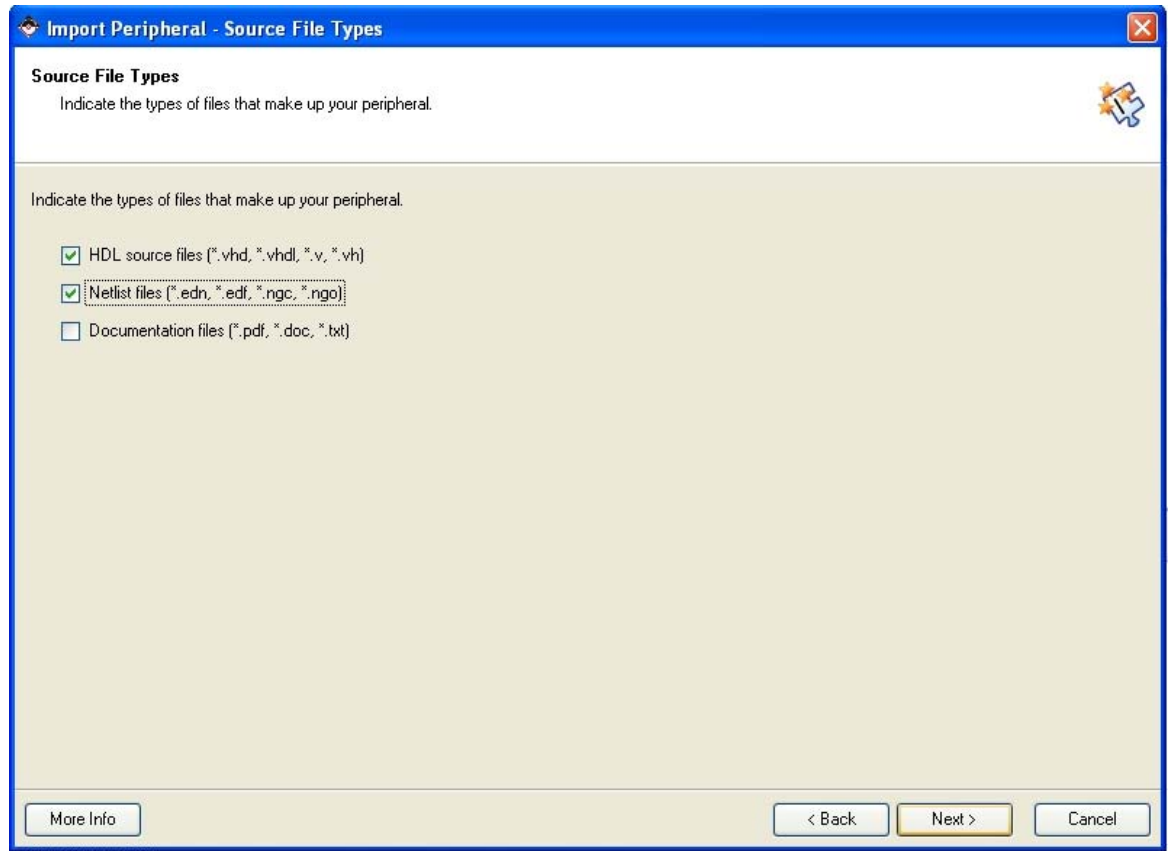

Check the "HDL source files" and "Netlist files" options, and click "Next".

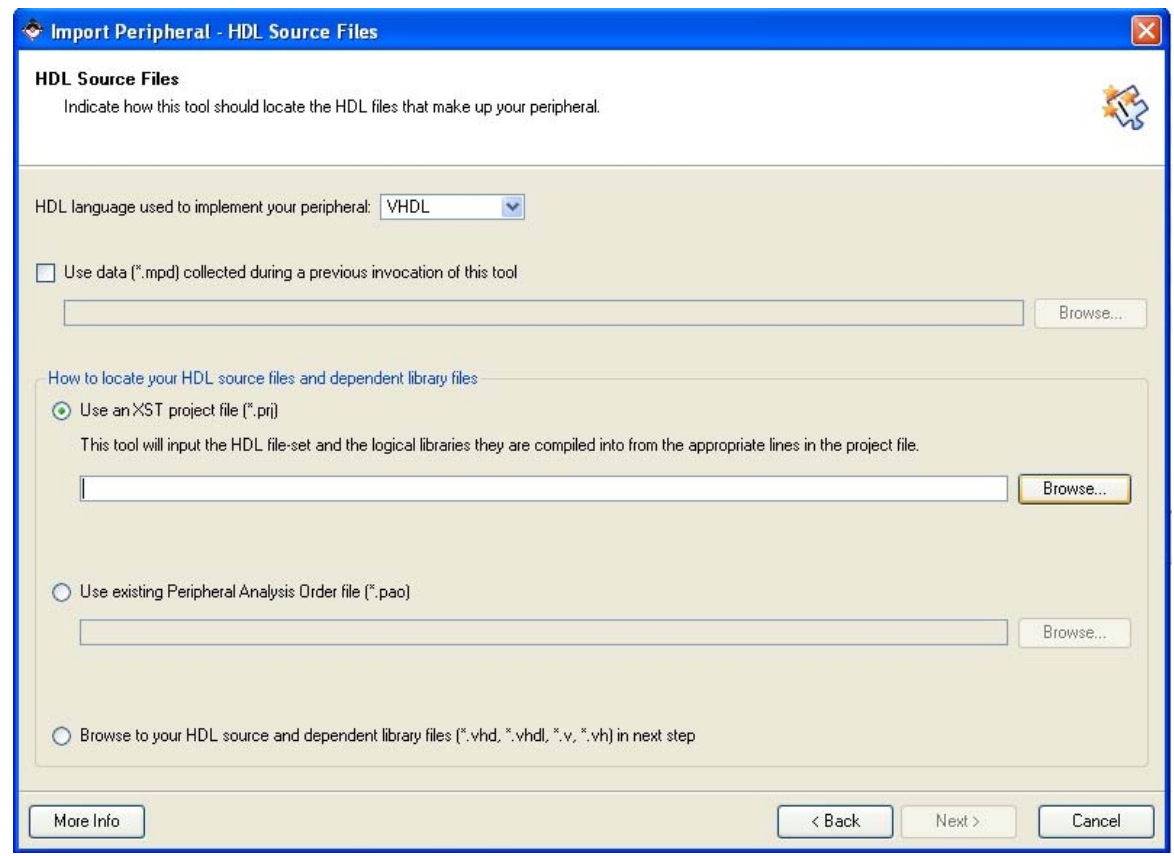

Select "Use an XST project file (*.prj)", and click on "Browse..." 


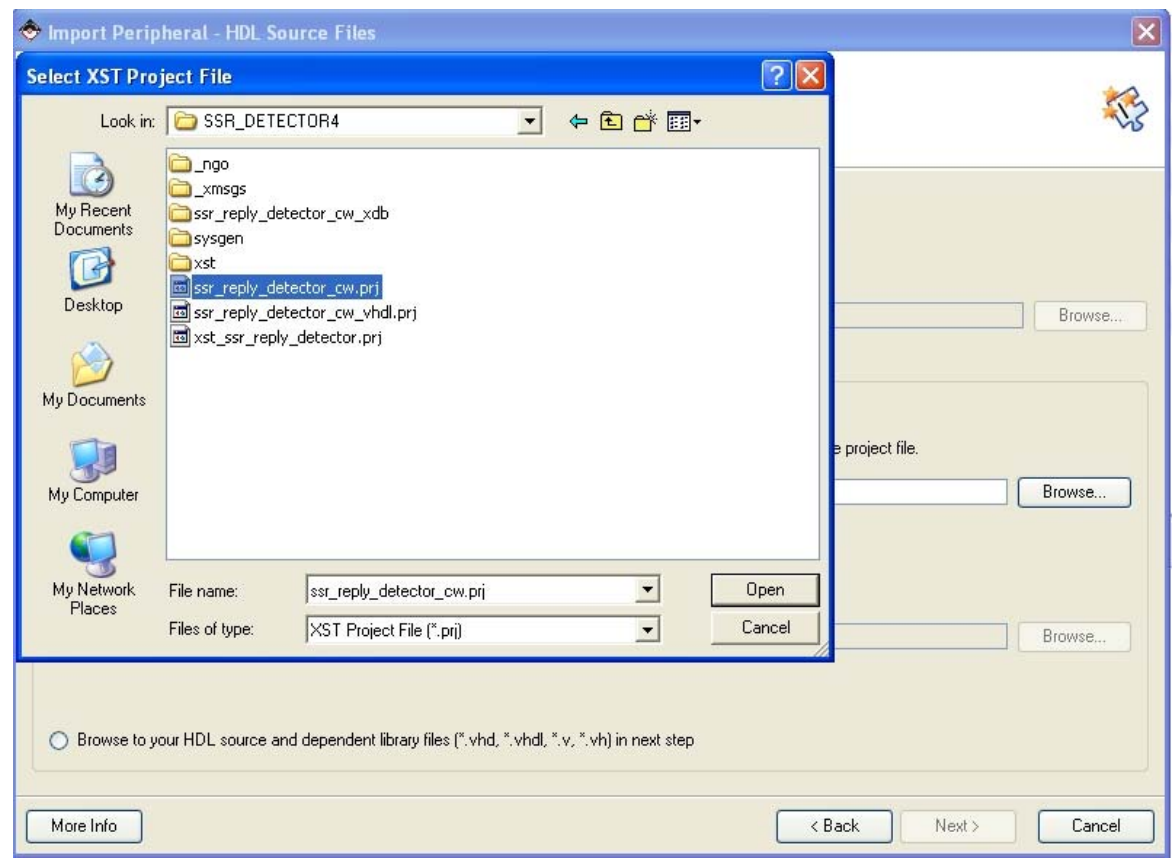

Navigate to the directory where you had Xilinx System Generator save the generated VHDL files and associated project files. Select "ssr_reply_detector_cw.prj”. Click "Open". Click "Next".

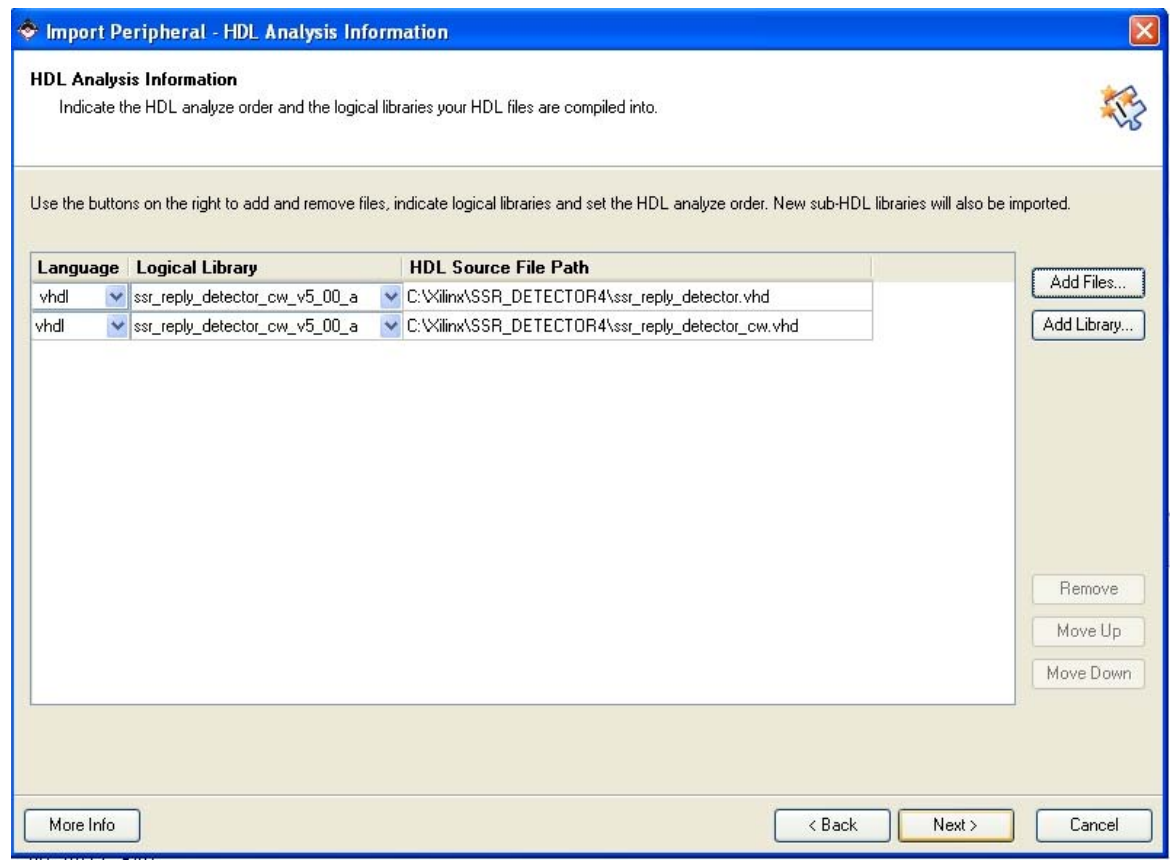

The VHDL files associated with the detector hardware should be visible now. Click "Next". 


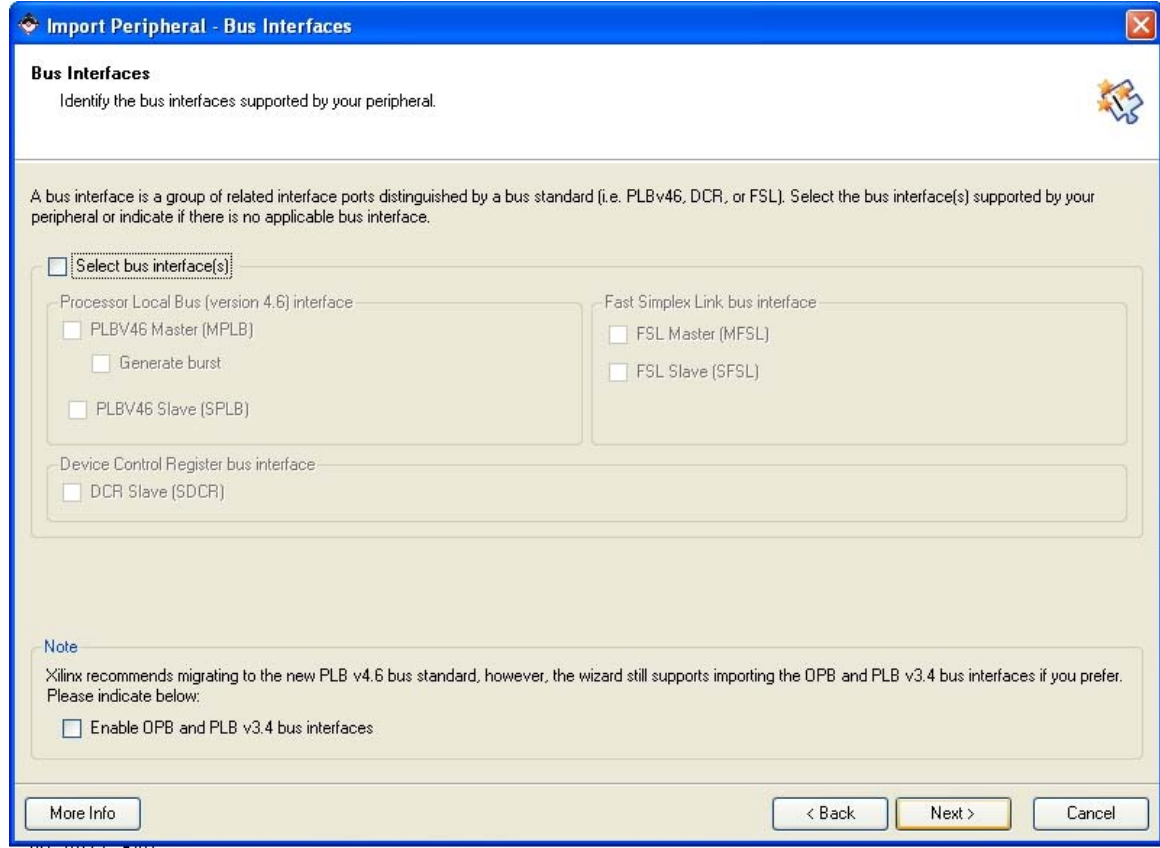

Uncheck "Select bus interface(s)" and then click "Next".

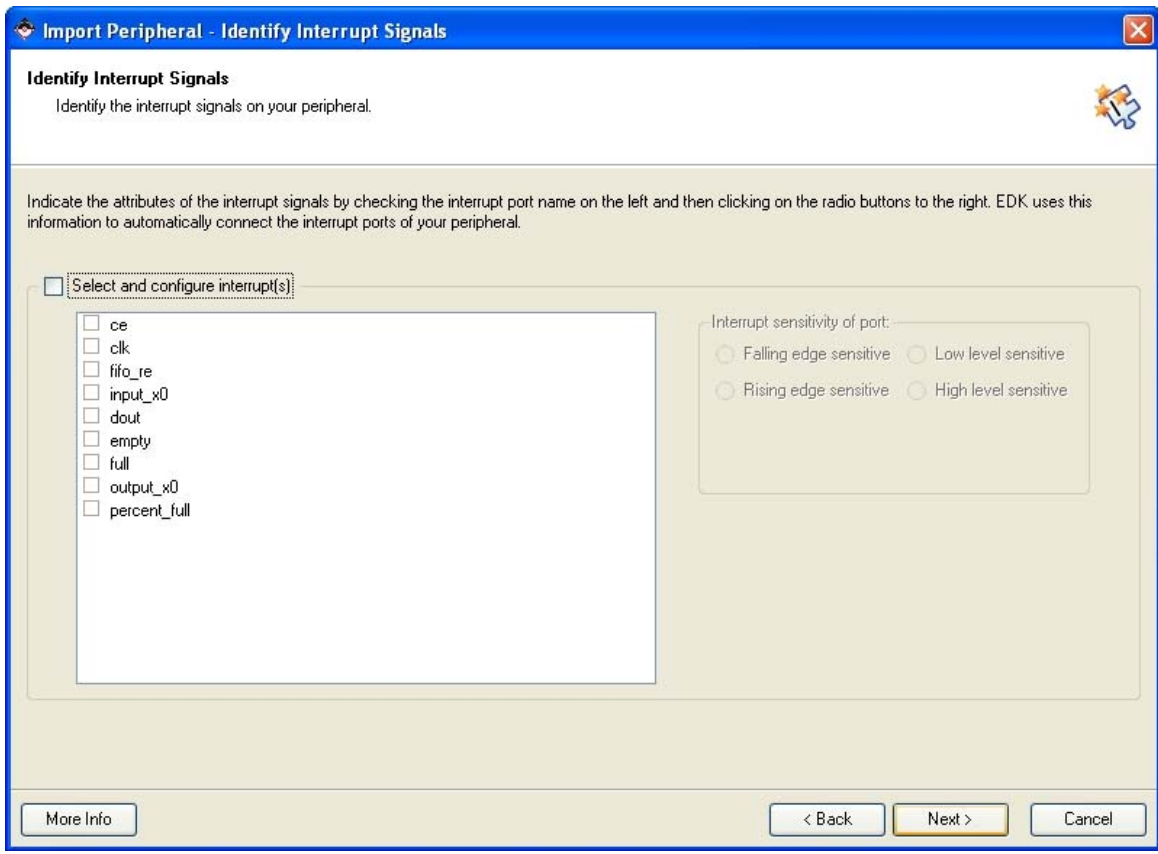

Uncheck "Select and configure interrupt(s)" and click "Next". 


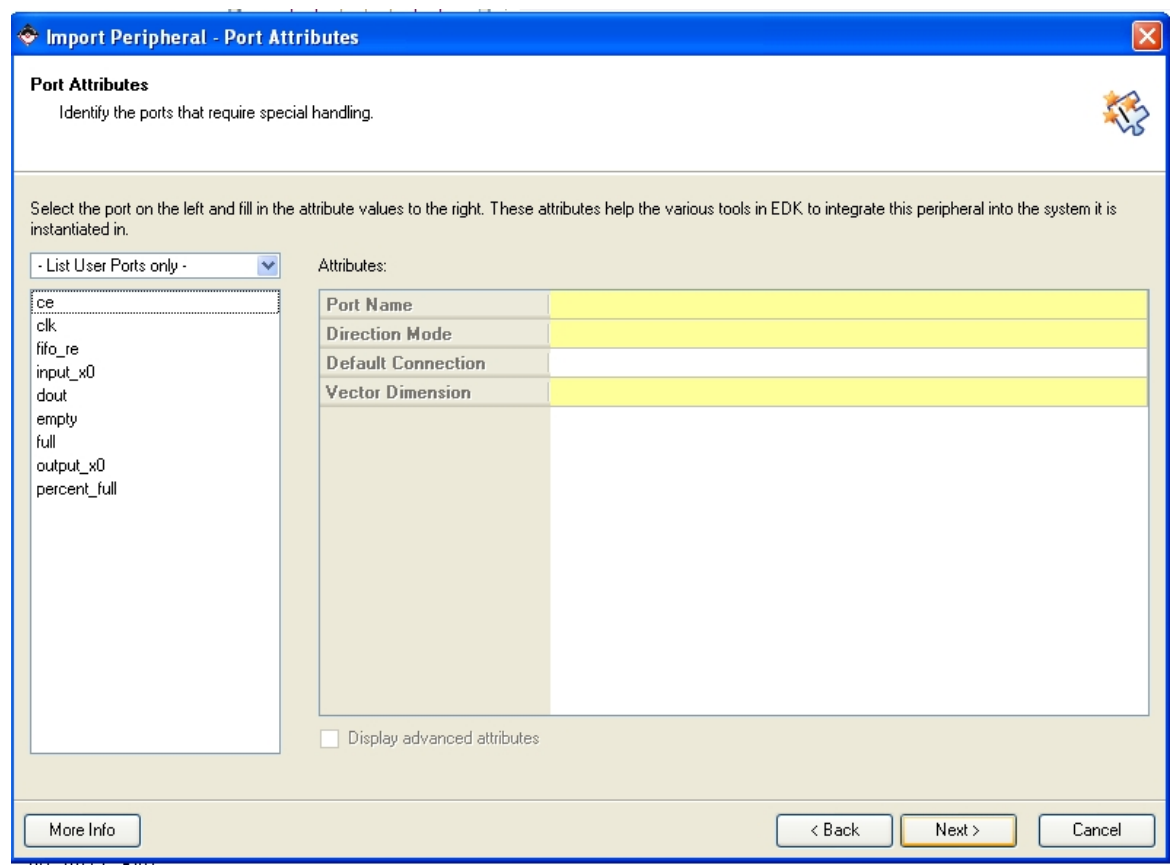

This screen shows the ports of the peripheral device. Click "Next".

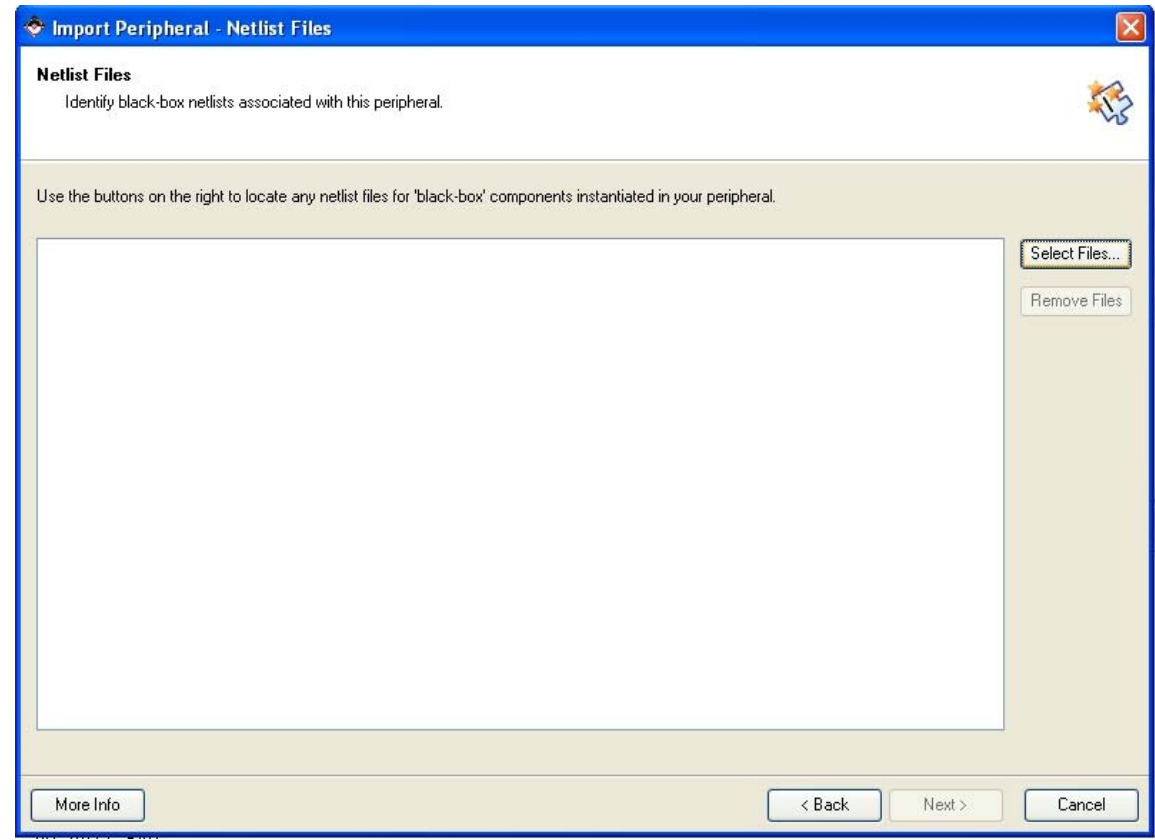

Click on "Select Files..." to add black-box modules to the peripheral device. These are used for elements of the detector such as delay blocks and the FIFO. 


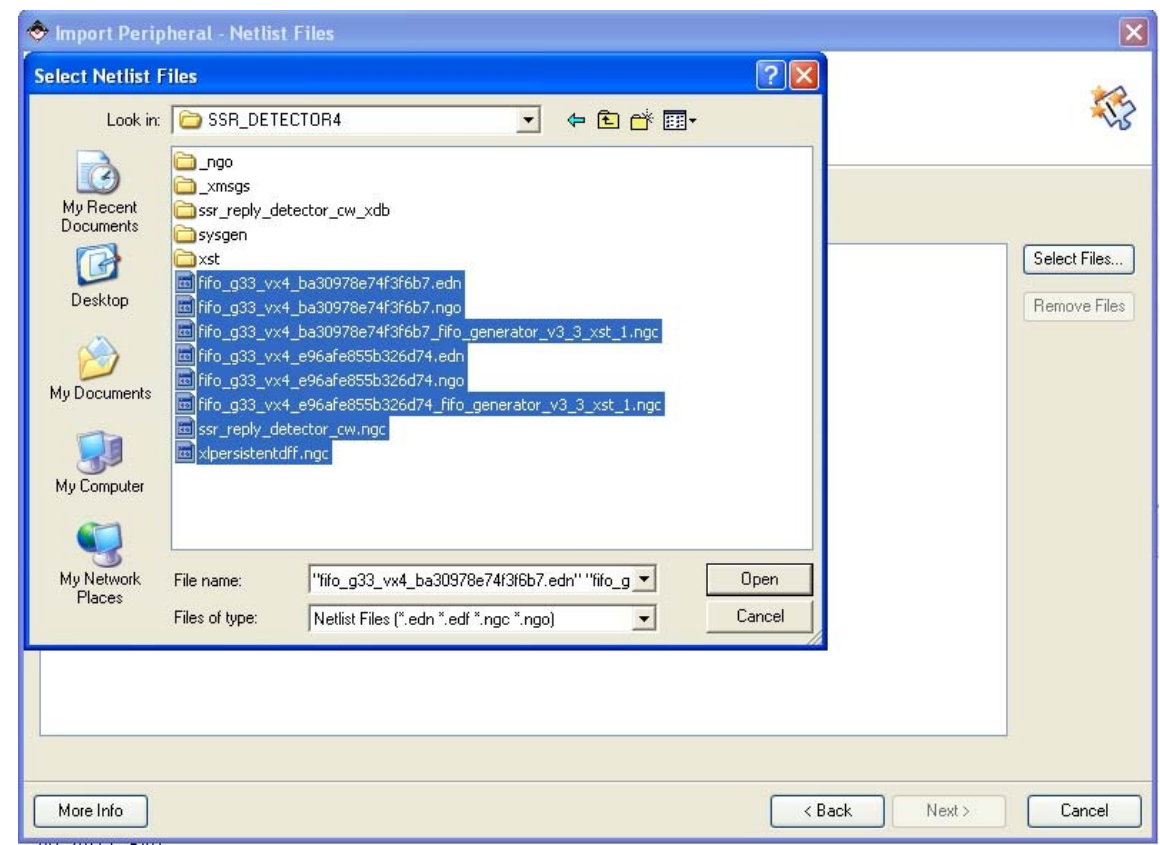

Navigate to the folder where your HDL detector modules are saved and select all the available *.edn, *.ngo, and *.ngc files. Click "Open" then click "Next".

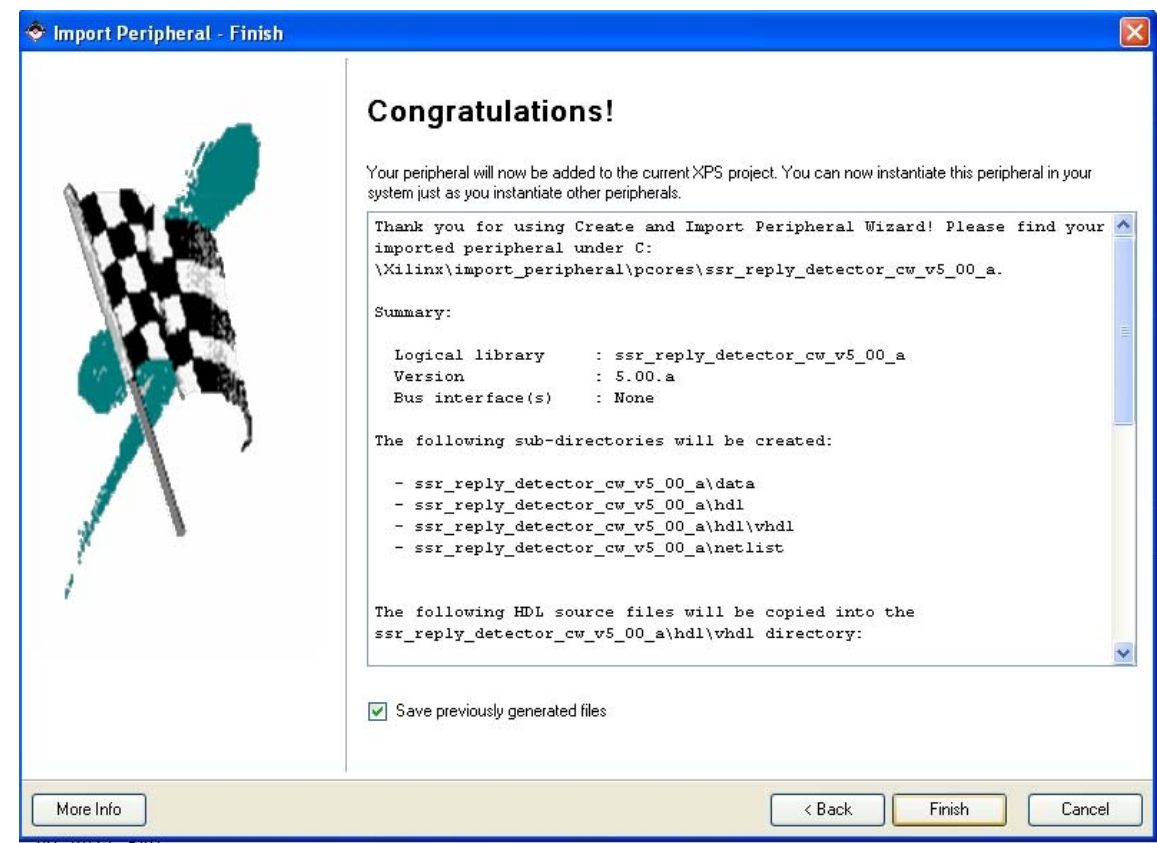

The custom peripheral is now created. Click "Finish". 


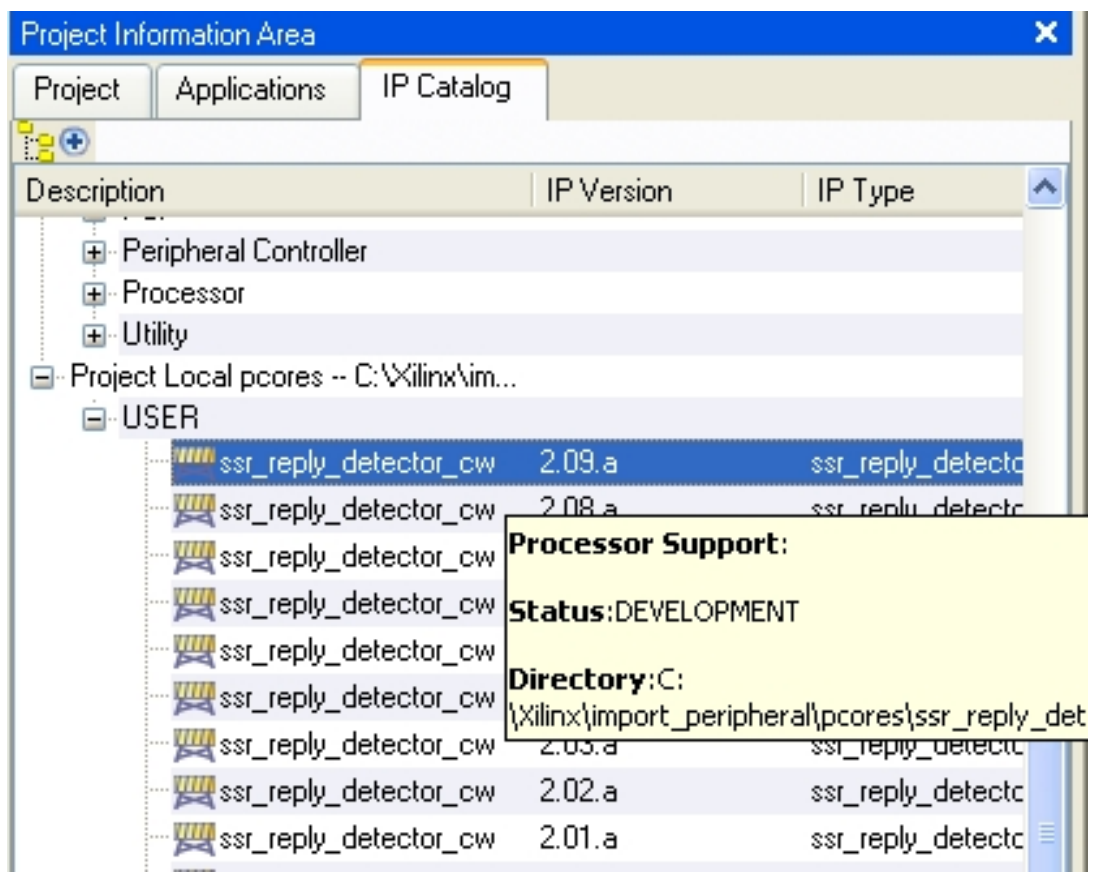

Add the new custom peripheral to your project by opening the "IP Catalog" tab in the "Project Information Area" frame. Double click on the newly created peripheral to add it to the project. 


\section{Step 6: Make Necessary Connections in the Xilinx Platform Studio EDK}

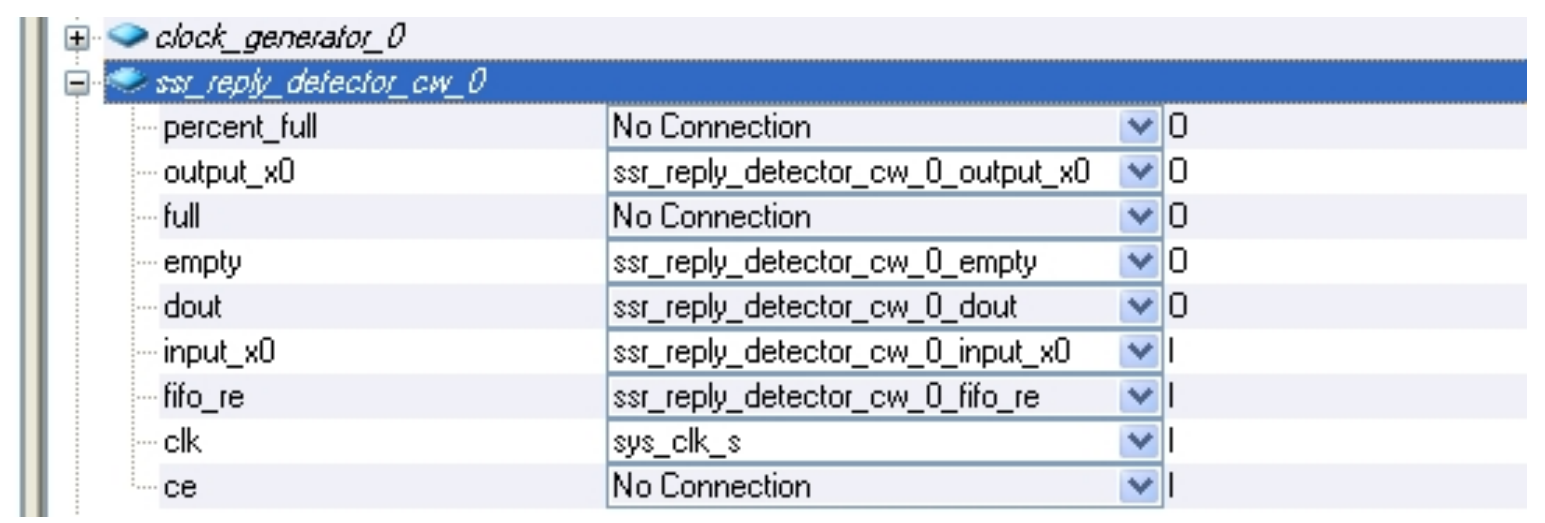

In the "Ports" list of the "System Assembly View" tab in Xilinx Platform Studio, expand the tab for the custom detector peripheral. In this case the peripheral is called "ssr_reply_detector_cw_0".

For the following ports, click on the dropdown menu and select "Make External":

output_x0

input_x0

For the following ports, click on the dropdown menu and select "New Connection":

empty
dout
fifo_re

Click on the dropdown menu for clk, and select "sys_clk_s".

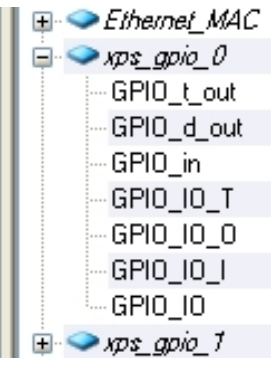

\begin{tabular}{|c|c|c|}
\hline No Connection & $\checkmark 0$ & [0:(C_GPIO_WIDTH-1)] \\
\hline No Connection & $\vee 0$ & [0:(C_GPIO_WIDTH-1)] \\
\hline No Connection & $\checkmark 1$ & [0:(C_GPIO_WIDTH-1)] \\
\hline No Connection & $\checkmark 0$ & [0:(C_GPIO_WIDTH-1)] \\
\hline ssi_reply_detector_cw_0_fifo_re & $\checkmark 0$ & [0:(C_GPIO_WIDTH-1)] \\
\hline No Connection & $\checkmark 1$ & [0:(C_GPIO_WIDTH-1)] \\
\hline No Connection & $\checkmark 10$ & [0:(C_GPIO_WIDTH-1] \\
\hline
\end{tabular}

Expand the xps_gpio_0 object in the "Ports" list. Click on the dropdown for "GPIO_IO_O" and attach to "ssr_reply_detector_cw_0_fifo_re". 


\begin{tabular}{|c|c|c|c|}
\hline Oxps goio 7 & & & \\
\hline GPI02_t_out & No Connection & $\vee 0$ & [0:(C_GPIO_WIDTH-1]] \\
\hline ...GPI02_d_out & No Connection & $\checkmark 0$ & [0:[C_GPIO_WIDTH-1]] \\
\hline GPIO2_in & Ssi_reply_detector_cW_0_empty & $\nabla 1$ & [0:[C_GPIO_WIDTH-1]] \\
\hline GPI02_IO_T & No Connection & $\checkmark 0$ & [0:[C_GPIO_WIDTH-1]] \\
\hline ....GPI02_IO_0 & No Connection & $\vee 0$ & [0:(C_GPIO_WIDTH-1]] \\
\hline ....GPI02_I0_I & ssi_reply_detector_cw_0_empty & $\checkmark 1$ & [0:(C_GPIO_WIDTH-1)] \\
\hline GPI02_I0 & No Connection & $\vee 10$ & [0:(C_GPIO_WIDTH-1]] \\
\hline GPIO_t_out & No Connection & $\checkmark 0$ & [0:(C_GPIO_WIDTH-1]] \\
\hline .... GPIO_d_out & No Connection & $\vee 0$ & [0:(C_GPIO_WIDTH-1]] \\
\hline GPIO_in & ssi_reply_detector_cw_0_dout & $\checkmark 1$ & [0:(C_GPIO_WIDTH-1]] \\
\hline GPIO_IO_T & No Connection & $\vee 0$ & [0:(C_GPIO_WIDTH-1)] \\
\hline GPIO_IO_0 & No Connection & $\checkmark 0$ & [0:(C_GPIO_WIDTH-1]] \\
\hline GPIO_IO_I & ssi_reply_detector_cw_0_dout & $\vee 1$ & [0:[C_GPIO_WIDTH-1]] \\
\hline GPIO_IO & No Connection & $\vee 10$ & [0:(C_GPIO_WIDTH-1]] \\
\hline
\end{tabular}

Expand the tab for "xps_gpio_1" in the "Ports" list. GPIO_1 is a dual channel GPIO - if the second channel is not shown, make GPIO_1 dual channel in its properties dialog. Make the following connections:
GPIO2_in :
ssr_reply_detector_cw_0_empty
GPIO2_IO_I :
ssr_reply_detector_cw_0_empty
GPIO_in :
GPIO_IO_I :
ssr_reply_detector_cw_0_dout
ssr_reply_detector_cw_0_dout

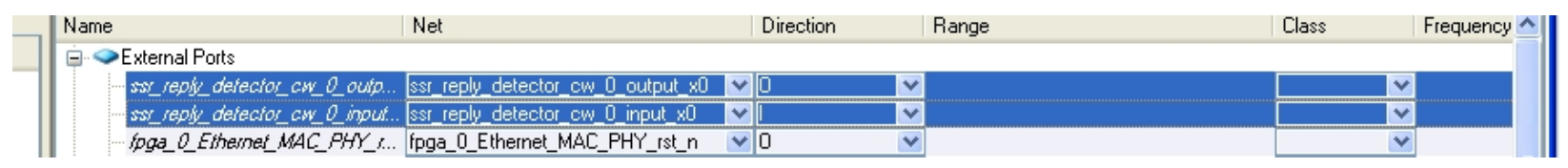

Expand the "External Ports" tab in the "Ports" list and make sure the following connections are made:
ssr_reply_detector_cw_0_output_x0_pin :
ssr_reply_detector_cw_0_output_x0
ssr_reply_detector_cw_0_input_x0_pin :
ssr_reply_detector_cw_0_input_x0 


\section{Step 7: Generate Hardware Image}

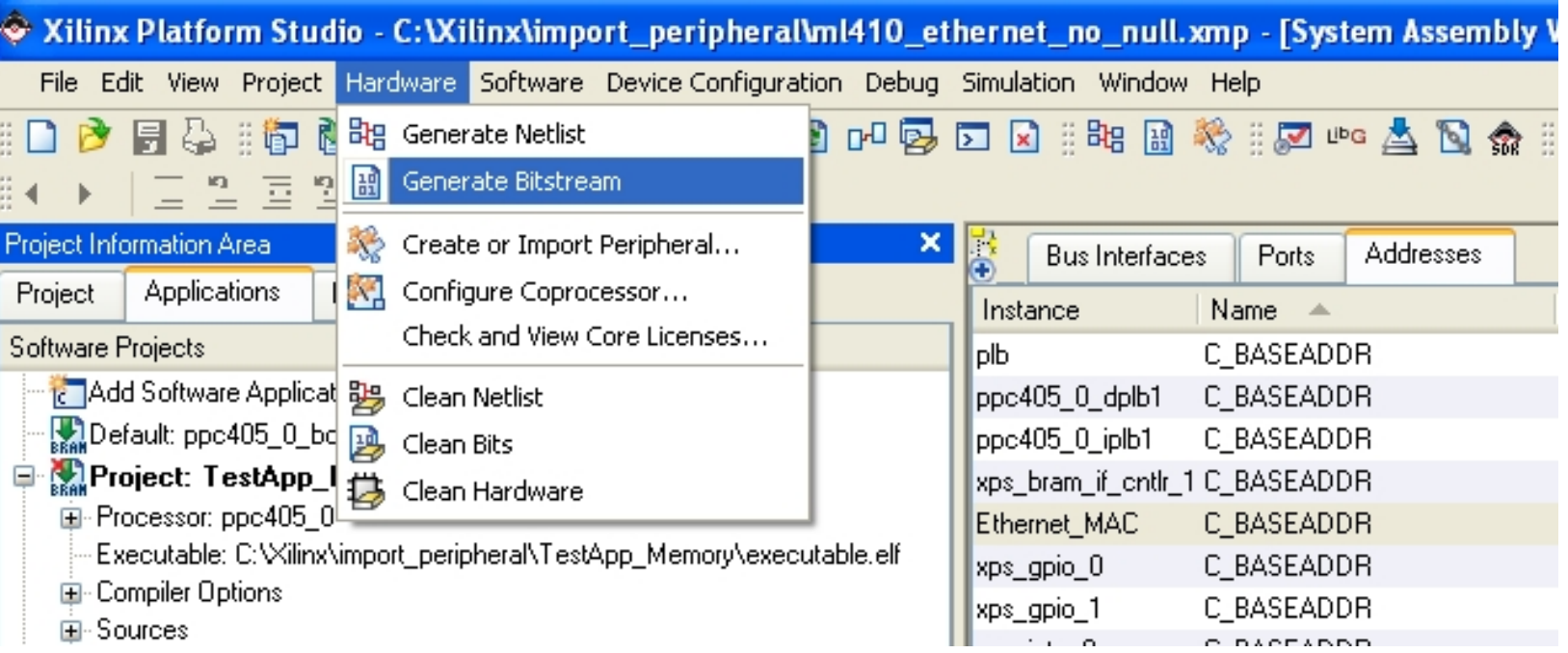

Once the system connections are all complete, generate the hardware bitstream by navigating to the "Hardware" menu in Xilinx Platform Studio and clicking on "Generate Bitstream".

\section{Step 8: Program the Device}

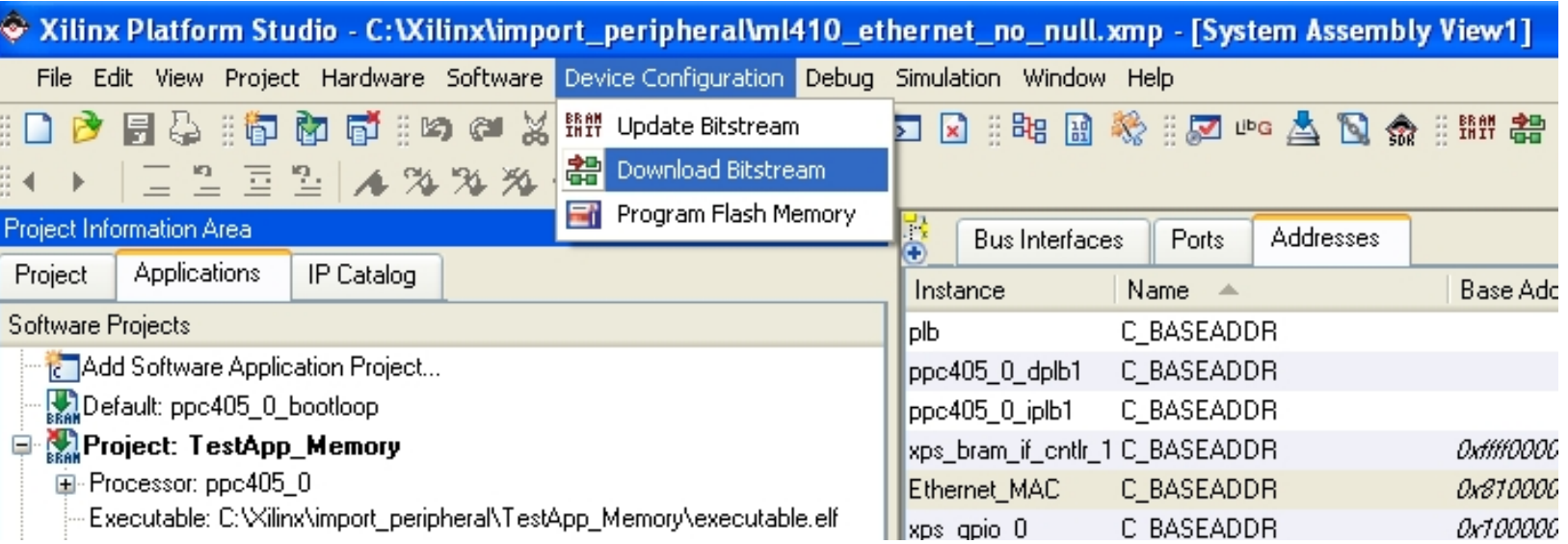

To program the FX60 chip with the generated bitstream, navigate to the "Device Configuration" menu in Xilinx Platform Studio and click on "Download Bitstream". 


\section{Step 9: Develop Embedded Software}

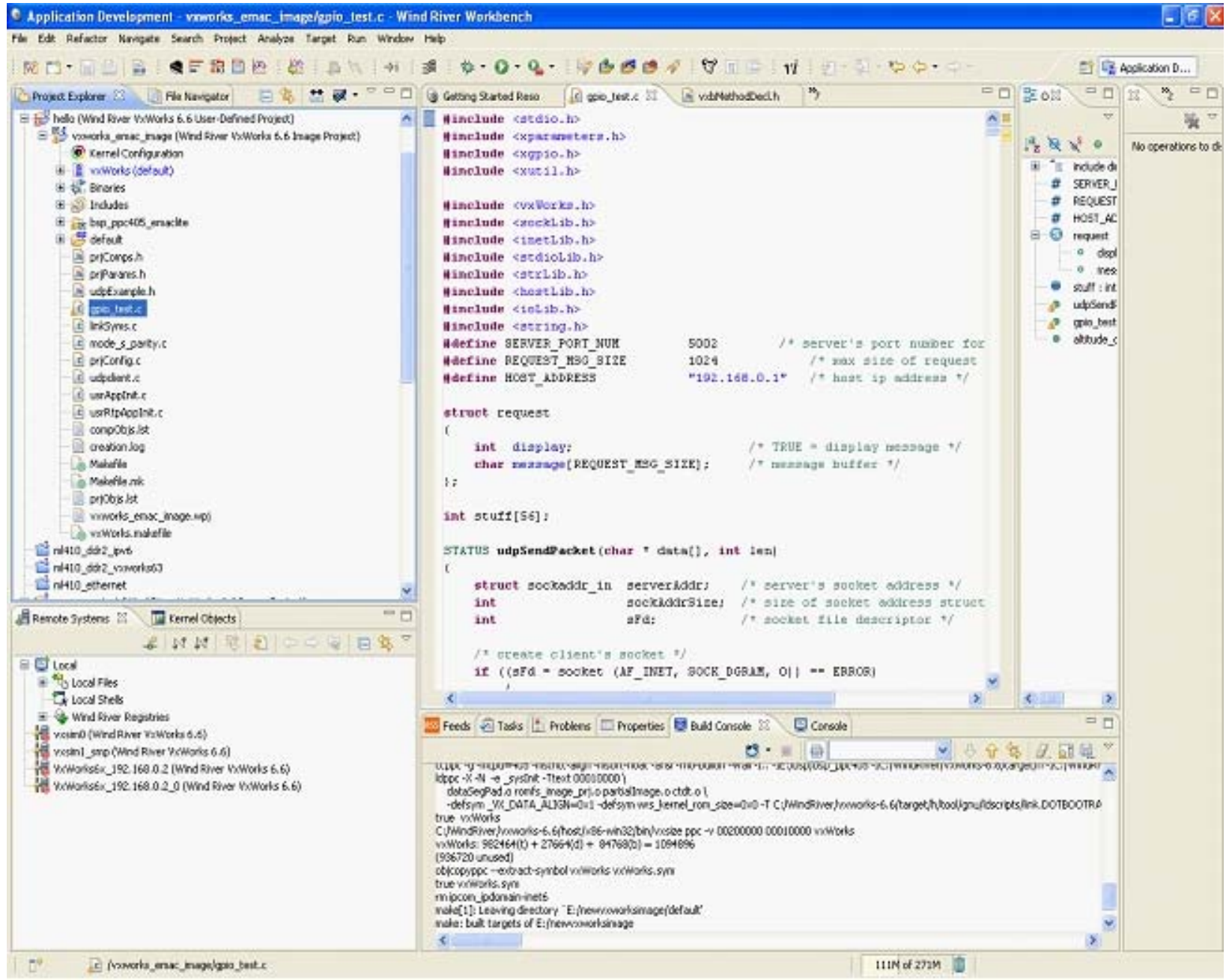

The software program used for testing is called "gpio_test.c". By creating any C file and loading it in with the VxWorks system, the program can then be run from the VxWorks command line. 


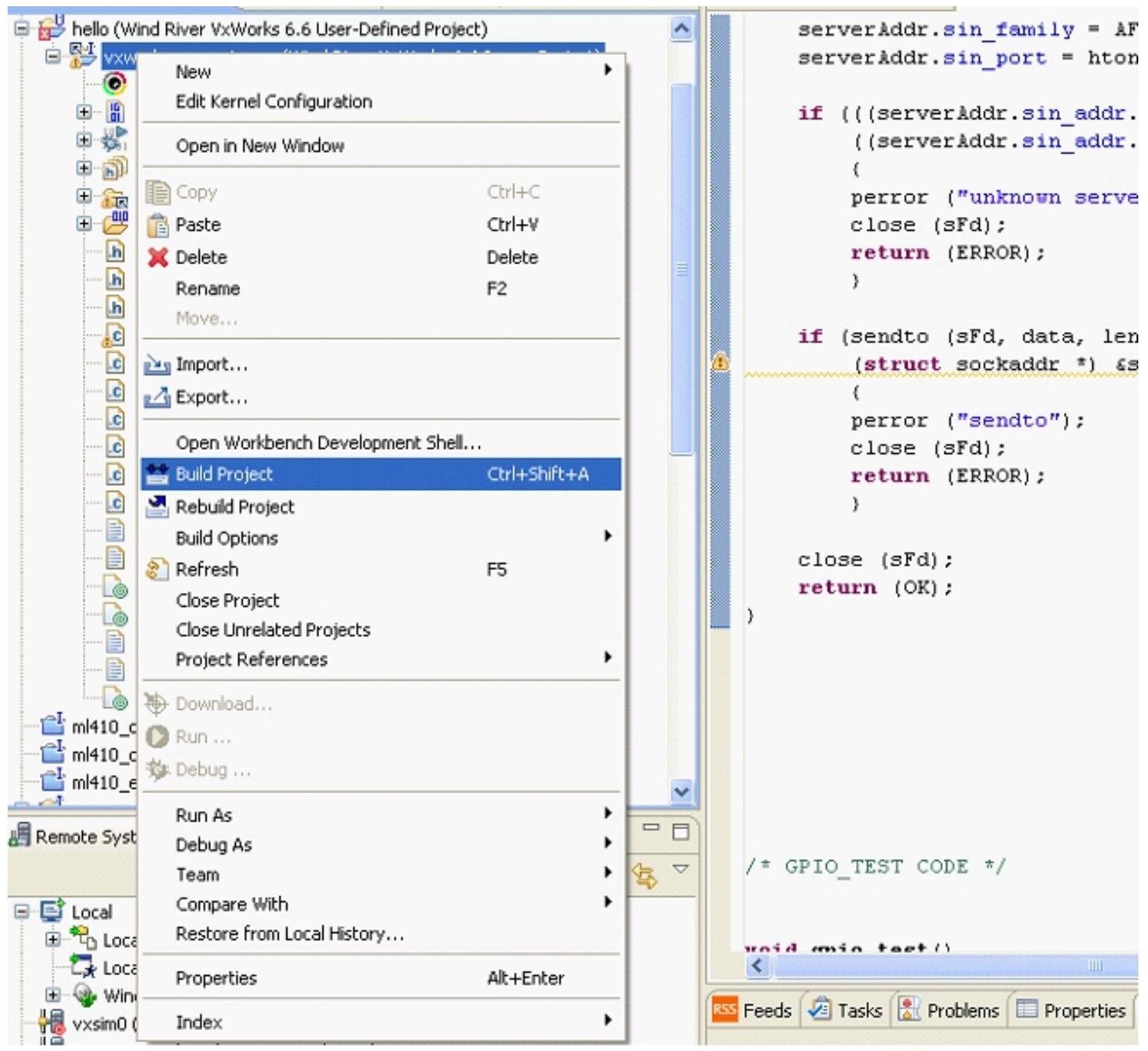

After editing the program or making any changes to the VxWorks system software, compile the system by right-clicking on the project (called "vxworks_emac_image") in the "Project Explorer" pane and clicking on "Build Project". 


\section{Step 10: Start the VxWorks Real-Time OS}

Start HyperTerminal to monitor the VxWorks terminal output over the serial port. Note on the Cal Poly Photonics lab computer, the Intel software running in the system tray must be shut down for the serial port to function correctly. Connect the serial cable to the right hand side DB9 serial port. Open a connection in HyperTerminal with 9600 baud 8-N-1.

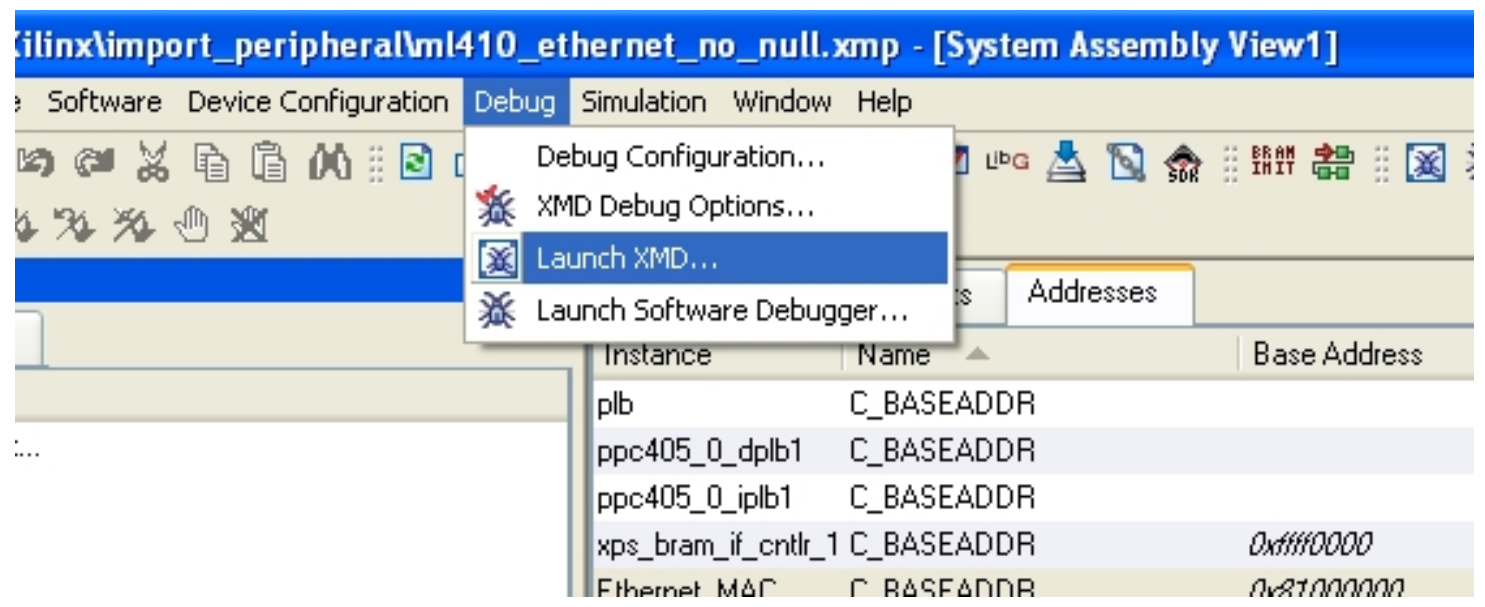

Open the XMD console window by navigating to the "Debug" menu in Xilinx Platform Studio and clicking on "Launch XMD...".

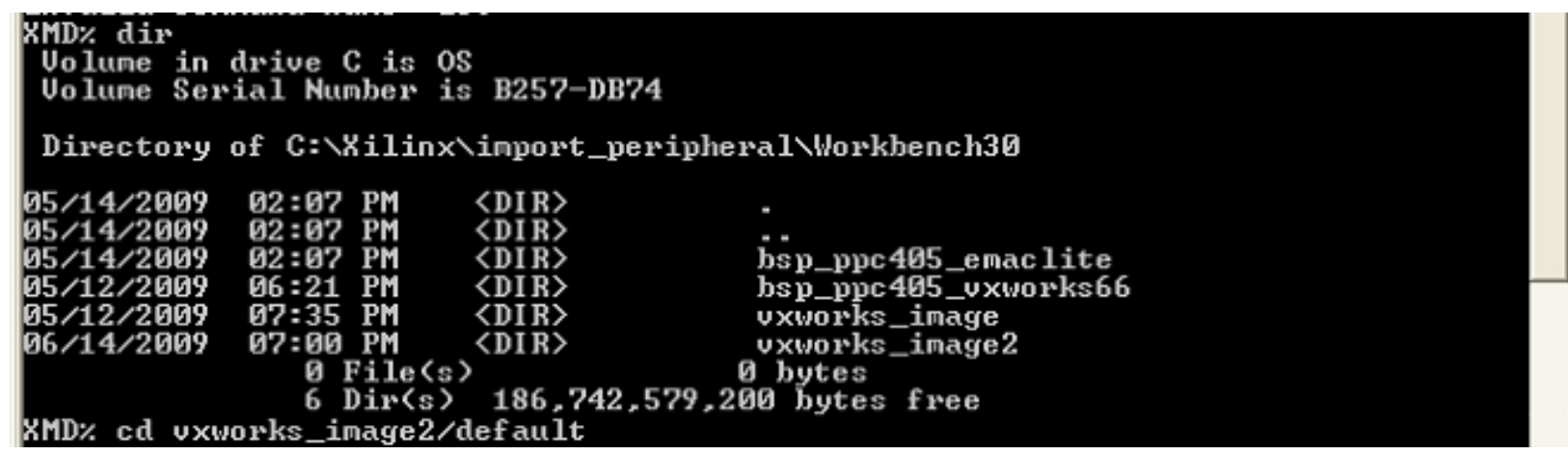

In the XMD console, the default directory should be the same as the Xilinx Platform Studio project. In this example, the current directory is "C: $|X i l i n x|$ import peripheral". Navigate to the subdirectory "C: $\mid X i l i n x \backslash$ import_peripheral $\backslash W o r k b e n c h 30 \backslash$ vxworks_image2 $\backslash$ default". 


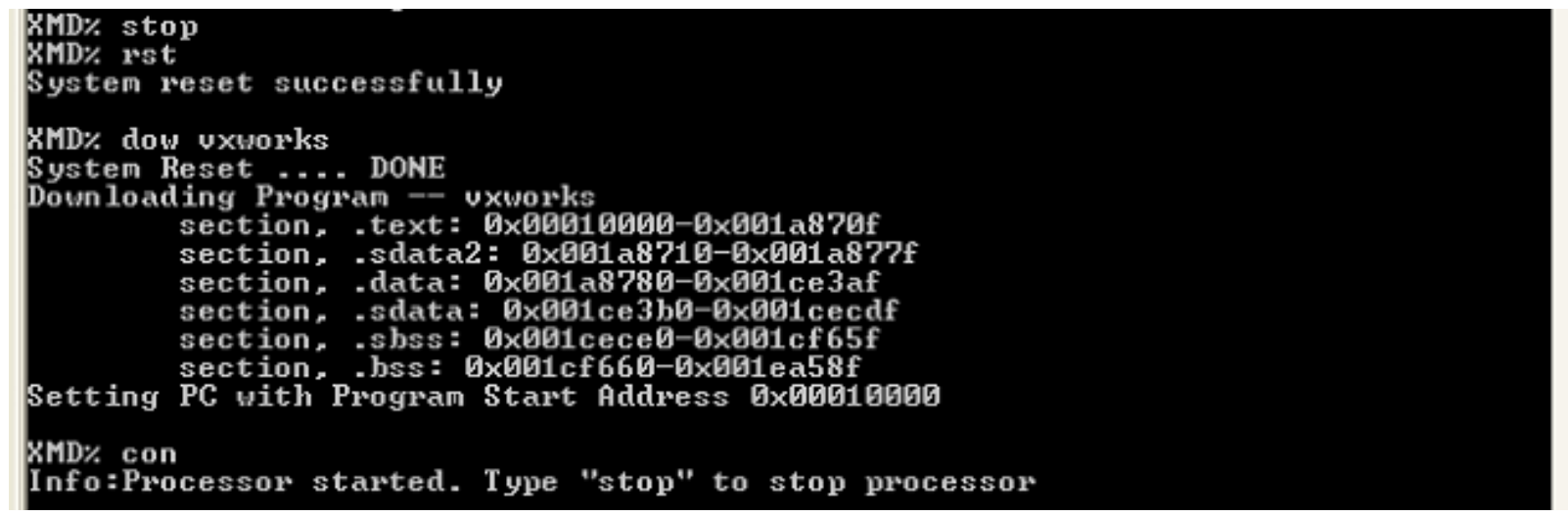

Type the command "stop" to stop any processes currently running on the PowerPC processor. Then, type the command "rst" to perform a system reset. Then issue the command "dow vxworks" to download the updated VxWorks image to the device (including any updated software). Finally, the command "con" starts the processor and the VxWorks operating system.

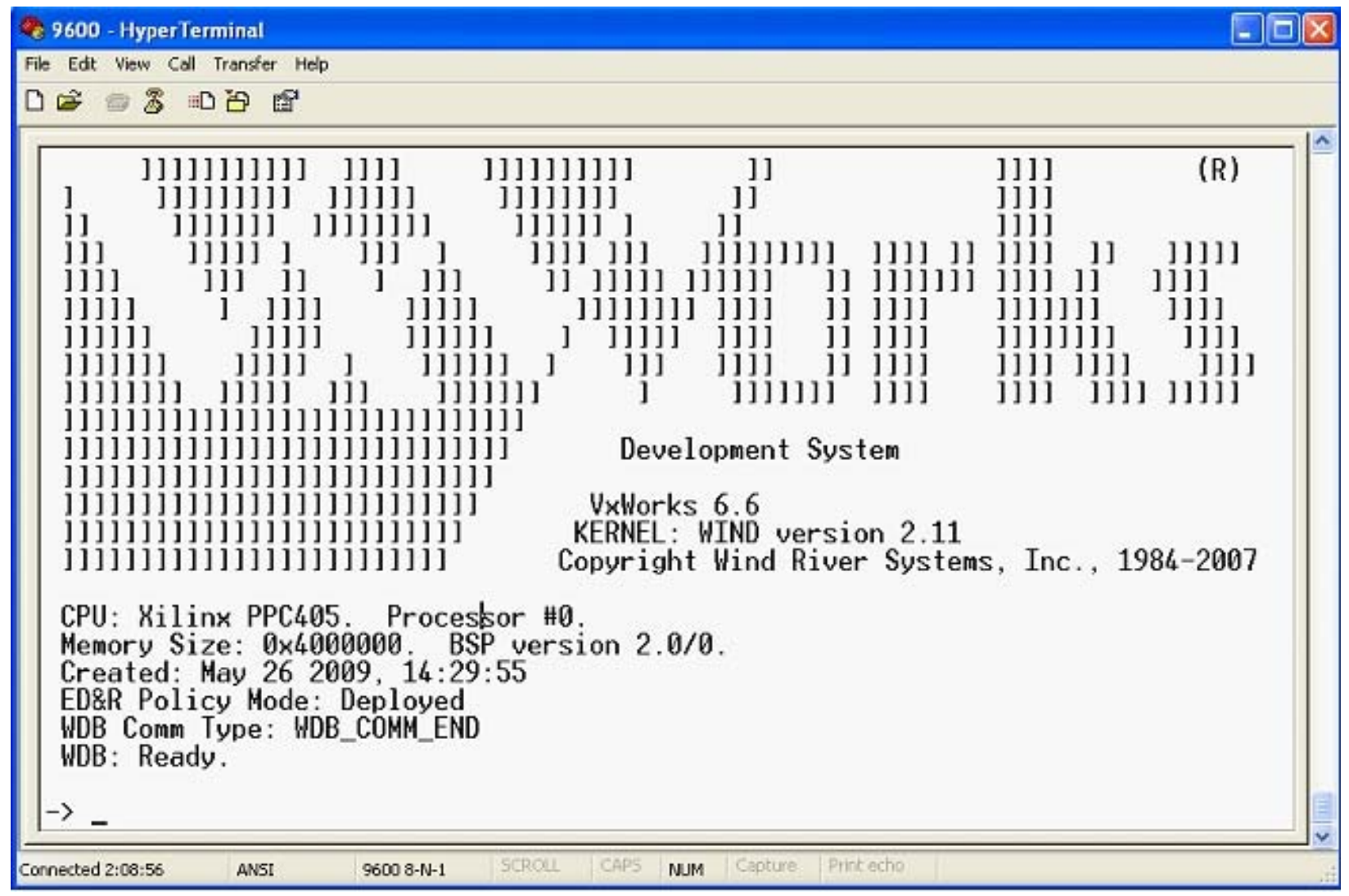

The HyperTerminal window should now display the VxWorks splash screen indicating a successful start-up. If nothing appears in the HyperTerminal window, try re-downloading the VxWorks kernel by repeating the steps in the XMD console starting with the "stop" command. 


\section{Step 11: Start the Custom Process in VxWorks}

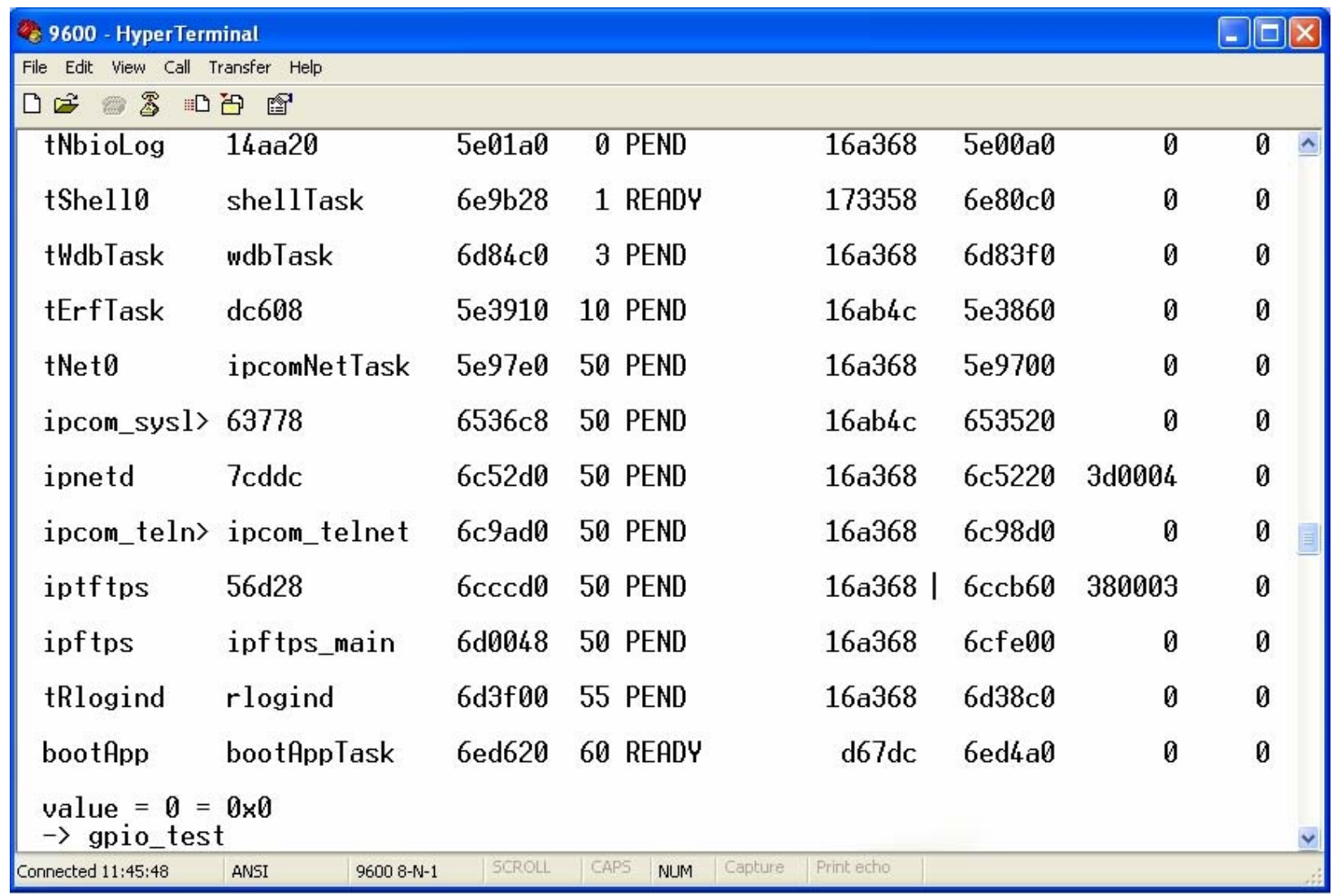

From the VxWorks console, accessed via either the serial interface or telnet, issue the name of the custom software module as a command. In our case this file is called "gpio_test". 


\section{Step 12: Verify Operation}

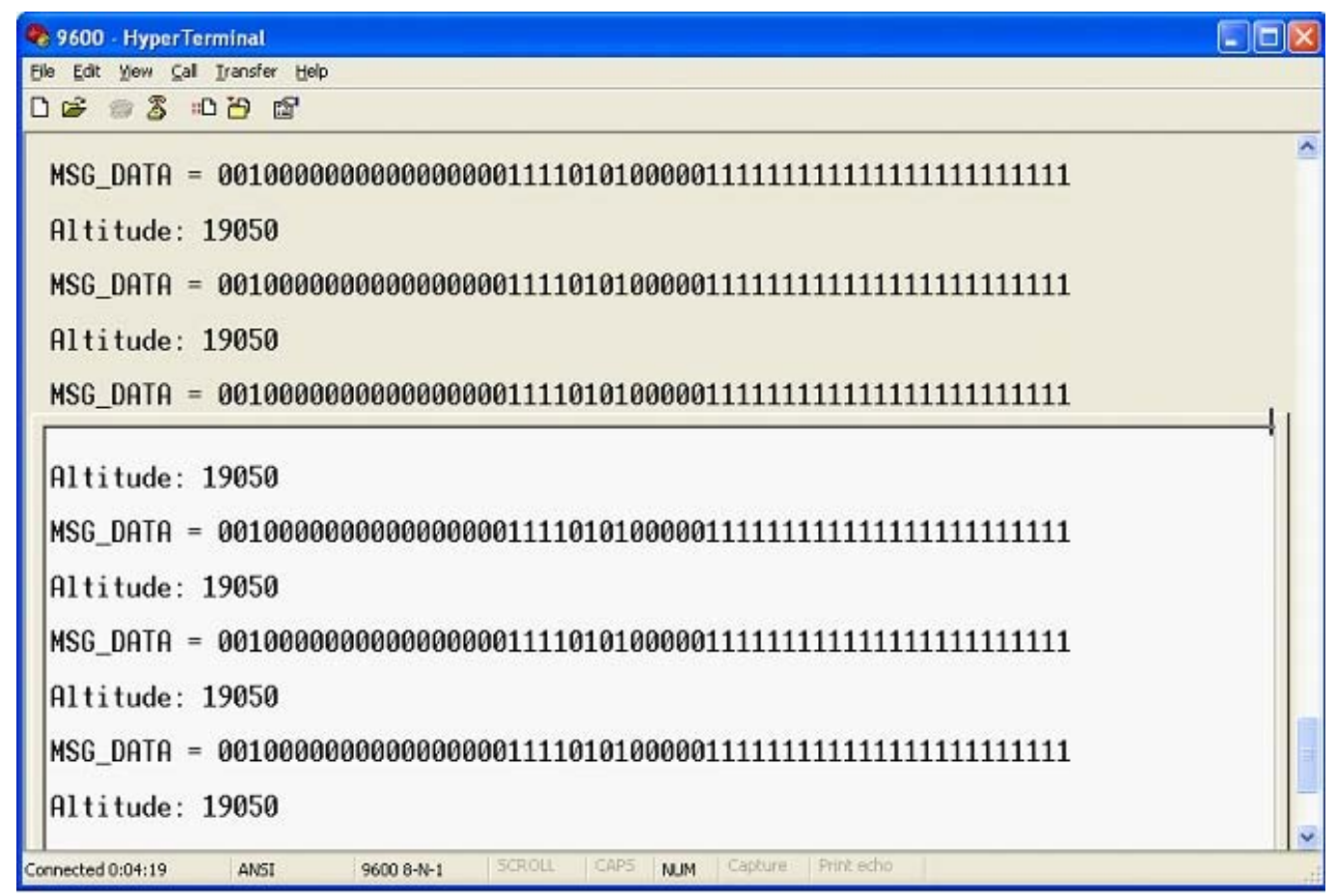

The software module should now start and poll the FIFO for data. When data is found in the memory, messages are read out and interpreted as altitude values. Each time one of these values is updated on the VxWorks console, a UDP packet containing the same information is sent over the Ethernet connection to the host PC.

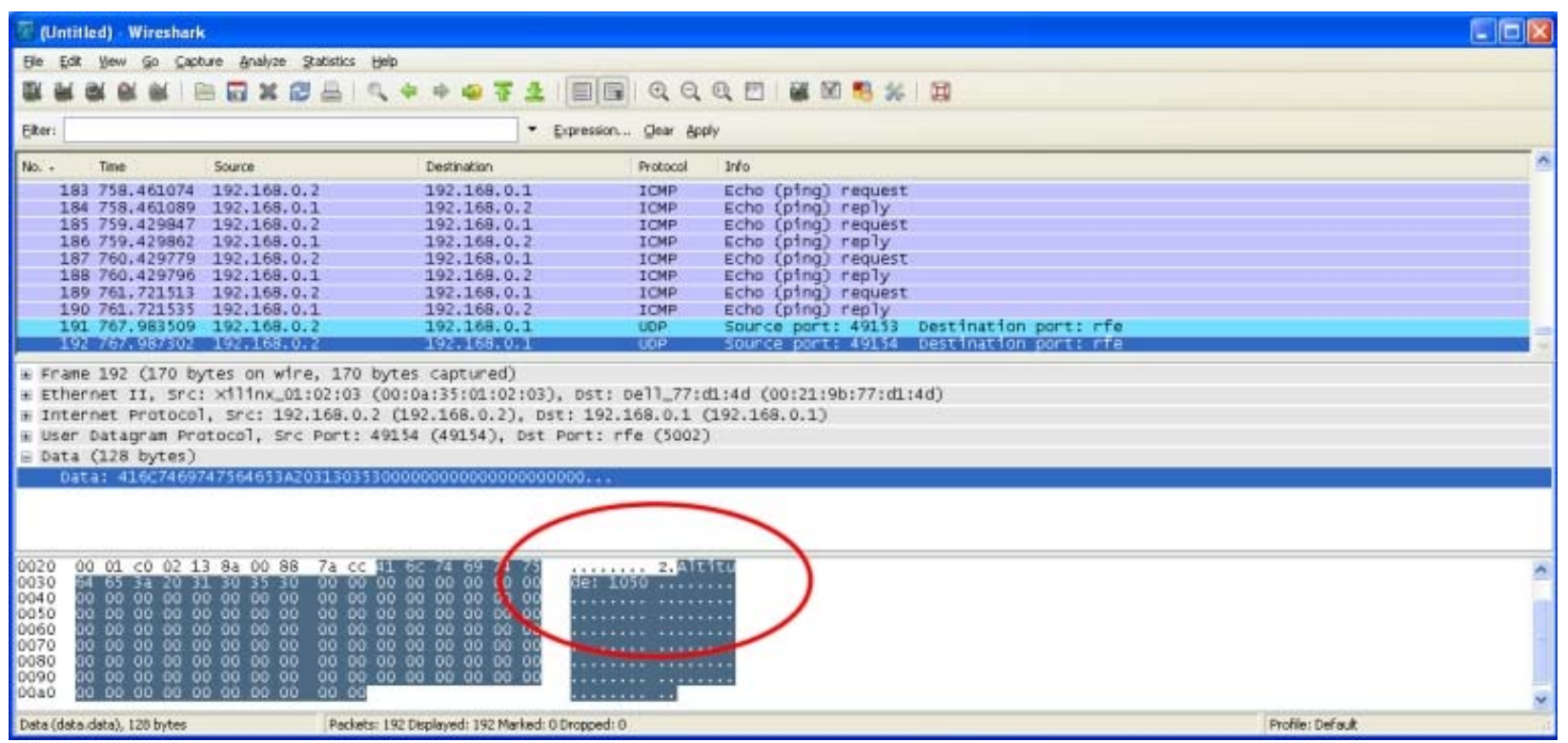

These UDP data packets can be observed on the host PC in the Wireshark network analyzer program. 


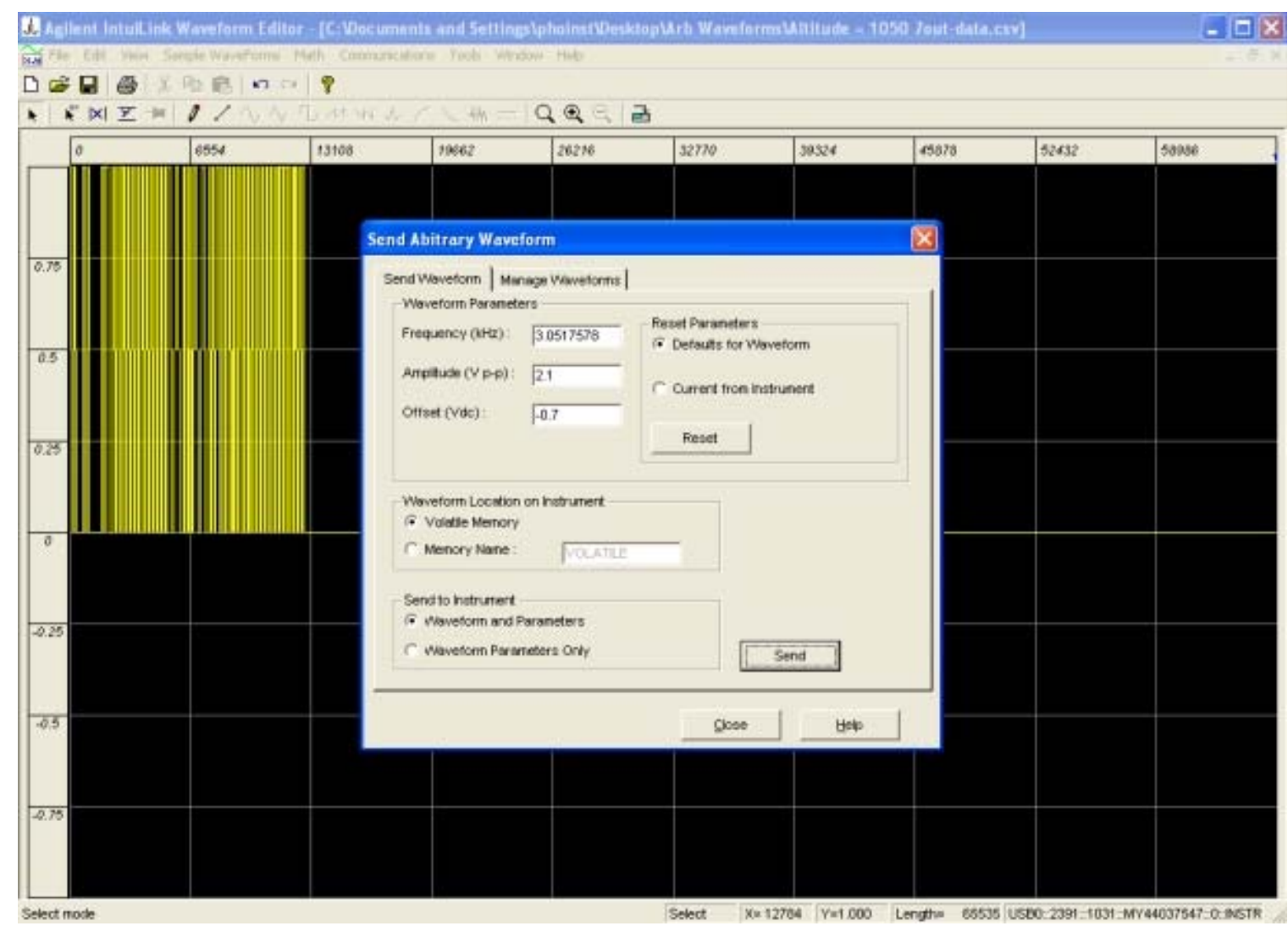

Power on the arbitrary waveform generator and start the Agilent IntuiLink Waveform Editor software. The device should be set to 2.1 volts peak-to-peak amplitude and -0.7 volts DC offset to get the logic levels within spec at the input terminal to the ML410. Operating frequency can be calculated as in the following example:

$0.01 \mu \mathrm{sec} /$ sample $* 65535 \mathrm{samples} /$ message $=655.36 \mu \mathrm{sec} / \mathrm{message}$

$1 / 655.36 \mu \mathrm{sec} /$ message $=\mathbf{1 . 5 2 5 8 7 8 9 1} \mathbf{~ k H z}$ 


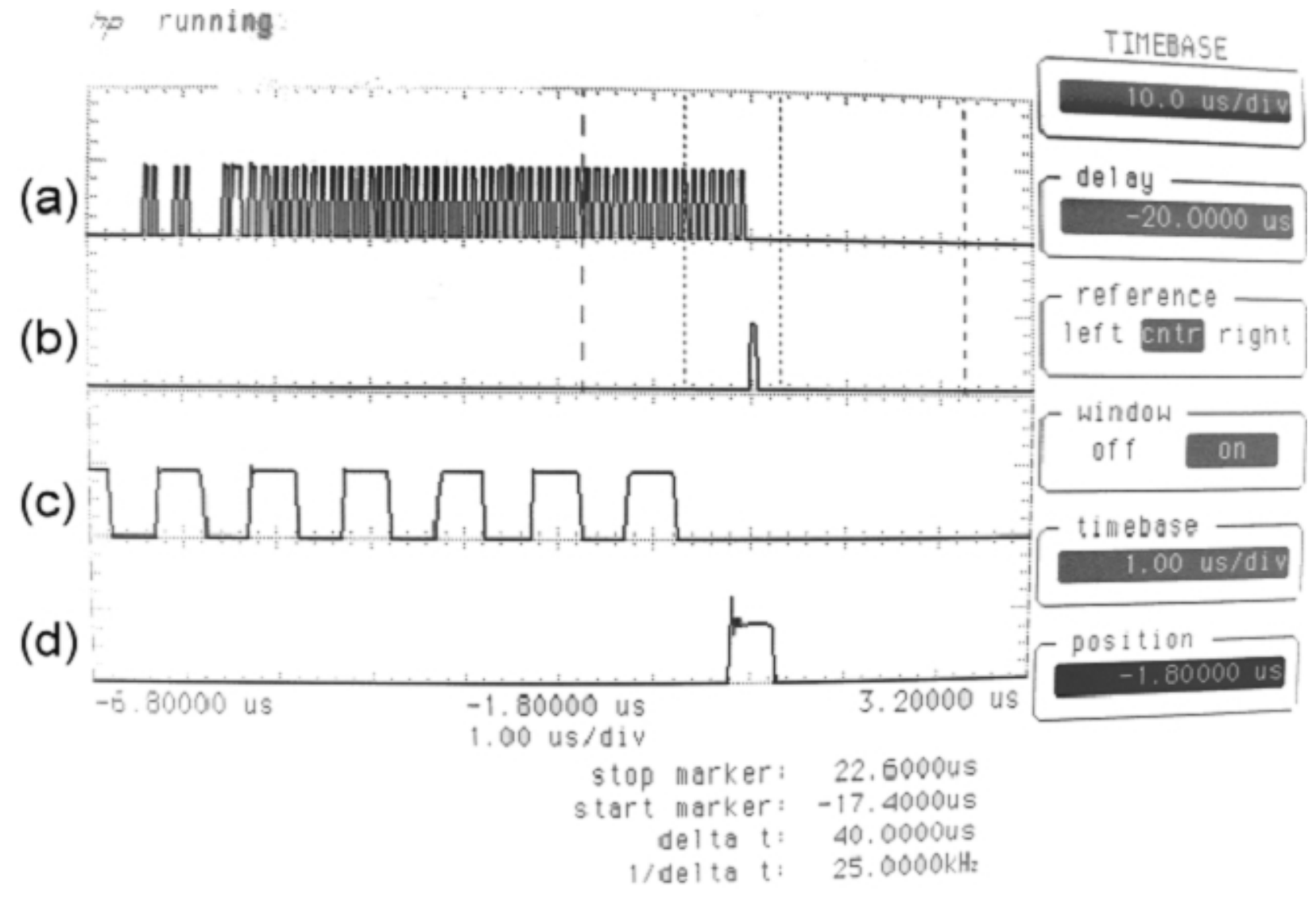

Additional verification of the design can be performed with the oscilloscope. Each time a transpond (a and c) is detected and data is saved into the FIFO, the "matched" signal ( $b$ and d) should go high. The "matched" signal is routed to the output SMA clock pin on the ML410 board and can be monitored on the oscilloscope. 